\title{
Calculation of the Deviatoric Stress Field in New Zealand
}

by

\section{Hamish Hirschberg}

A thesis submitted to Victoria University of Wellington in partial fulfilment of the requirements for the degree of Master of Science in Geophysics

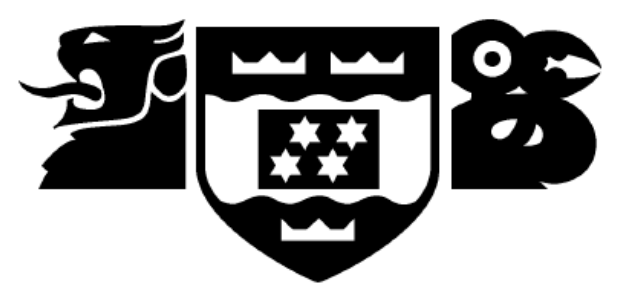

Institute of Geophysics

School of Geography, Environment and Earth Sciences

Victoria University of Wellington

Wellington, New Zealand

$28^{\text {th }}$ February 2017 



\section{Abstract}

I model the vertically averaged deviatoric stress field for New Zealand using velocity and crustal density data. I use a thin sheet model of a viscously deforming lithosphere, averaging over a depth of $100 \mathrm{~km}$ and solve the stress balance equation. Two methods of solving the stress balance equation are compared: one method solves first for deviatoric stresses due to gravitational potential energy per unit volume before accounting for deviatoric stresses due to boundary conditions; the other method assumes an isotropic viscosity to relate deviatoric stress to strain rate, solving for the viscosity field. Under synthetic testing, the two step method is able to cope with high levels of noise but contains edge effects. The method solving for viscosity is accurate at low noise levels, however, it is unreliable at high noise levels. I apply the two step method to New Zealand using a Quaternary and a GPS-derived velocity model. Vertically averaged deviatoric stress magnitudes are found to be 10$30 \mathrm{MPa}$, similar to magnitudes found for other plate-boundary zones. Gravitational and boundary stresses each account for approximately half of the full deviatoric stress. Effective viscosities are found to be $1-10 \times 10^{21} \mathrm{~Pa} \mathrm{~s}$ in the regions of most active deformation, which can be interpreted in terms of the long term strength of the lithosphere controlled by temperature and/or lithology. 


\section{Acknowledgements}

There are several people whose help during this project must be acknowledged. My supervisors, Simon Lamb and Martha Savage, have been instrumental in allowing me to complete this project. I and this project have greatly benefited from their guidance, help, and knowledge. I am grateful for the funding I have received through the Frank Evison and Victoria University of Wellington Master's Scholarships that has financially supported me through this project. Thanks are due to John Haines for his help implementing the boundary stress part of the stress method.

For numerous discussions throughout the year, both related and unrelated to this project, I would also like to thank my officemates, Rachel Barrett, Andy McNab, Stefan Mroczek, Nicolas Oestreicher (quelquefois en français), Sam Taylor-Offord, and Seb Thornton, as well as other members of the VUW Institute of Geophysics. Similarly, I thank Renée Tonkin for conversations from the other side of the world that have helped keep me sane.

Of course, I also thank my family, especially my parents, for the loving support and encouragement they have provided over many years. This has allowed me to gain the skills ultimately required for this project and supported me in carrying out this project. Mum, Dad, and Andrea deserve special thanks for this last year for the many things they have done for me and tolerating me whilst I have been living at home. 


\section{Contents}

Abstract $\quad$ i

Acknowledgements ii

Table of Contents iii

List of Figures vi vi vis

List of Tables vii

1 Introduction $\quad 1$

1.1 Motivation . . . . . . . . . . . . . . . . . . 1

1.2 Previous Work . . . . . . . . . . . . . . . . . . . 3

1.3 Tectonic Setting . . . . . . . . . . . . . . . . . . 3

1.4 Background Theory . . . . . . . . . . . . . . . . 6

1.4.1 Measuring Stress . . . . . . . . . . . . . 7

1.4.2 Plate Boundary Stress Field . . . . . . . . . . . . . 7

1.5 Definitions . . . . . . . . . . . . . . . . . 8

1.5.1 Summation Convention . . . . . . . . . . . . . . . 8

1.5.2 Stress Definitions . . . . . . . . . . . . . . 9

2 Method 12

2.1 Data . . . . . . . . . . . . . . . . . . . 12

2.1.1 Quaternary Velocity Field . . . . . . . . . . . . 13

2.1.2 GPS-derived Velocity Field . . . . . . . . . . . . . 13

2.1.3 Gravitational Potential Energy per Unit Volume . . . . . . . . 15 
2.2 Stress Models . . . . . . . . . . . . . . . . 16

2.2.1 Stress Balance Equation . . . . . . . . . . . . 16

2.2 .2 Stress Method . . . . . . . . . . . . . . . 18

2.2.3 Strain Rate Method . . . . . . . . . . . . . . . . . . . 21

3 Synthetic Testing 23

3.1 Testing Method . . . . . . . . . . . . . . . 23

3.2 Testing Results . . . . . . . . . . . . . . . . . . . . 24

3.3 Recovering Gravitational Stress Field . . . . . . . . . . . . . . . 26

4 Results $\quad 30$

4.1 Stress Results . . . . . . . . . . . . . . . . . . 32

4.1.1 Gravitational Stress Results . . . . . . . . . . . . . . . 32

4.1 .2 Total Stress Results . . . . . . . . . . . . . . . . . . . . 32

4.1.3 Boundary Stress Results . . . . . . . . . . . . . . . . 35

4.1 .4 Misfit . . . . . . . . . . . . . . . 36

4.2 Viscosity Results . . . . . . . . . . . . . . . . 37

$\begin{array}{lll}5 & \text { Discussion } & 39\end{array}$

5.1 Comparison to Similar Calculations . . . . . . . . . . . . . . . . . 39

5.2 Comparison to New Zealand Measurements . . . . . . . . . . . . . . . 40

5.2.1 Comparison between Models . . . . . . . . . . . . . . . . . 40

5.2.2 Comparison between Stress and Strain Rate Methods . . . . . 41

5.2.3 Comparison to Stress Directions . . . . . . . . . . . . . . . . . 42

5.3 Implications of Stress Magnitude . . . . . . . . . . . . . . . . . . . . 43

5.4 Rheology of the Deforming Zone . . . . . . . . . . . . . . . . . . . . 45

5.5 Conclusion . . . . . . . . . . . . . . . . . 47

$\begin{array}{ll}\text { Bibliography } & 48\end{array}$

A Codes Used $\quad 55$

A.1 Gravitational Stress . . . . . . . . . . . . . . . . . . 55

A.2 Basis Functions . . . . . . . . . . . . . . . . 56

A.3 Full Deviatoric Stress . . . . . . . . . . . . . . . . . 56 
A.3.1 Minimisation of Equation (2.11) . . . . . . . . . . 56

A.4 Full Stress Method Implementation . . . . . . . . . . . . . . . 57

B Complete Results $\quad 58$

B.1 Results of Quaternary Velocity Model . . . . . . . . . . . . . . . . . 59

B.2 Results of GPS-Derived Velocity Model . . . . . . . . . . . . . 65 


\section{List of Figures}

1.1 New Zealand tectonic setting . . . . . . . . . . . . . . . . . . 5

2.1 Comparison of velocity models . . . . . . . . . . . . . . . . . . . 14

3.1 Input fields for synthetic testing . . . . . . . . . . . . . . . . . . . 24

3.2 GPE fields for synthetic testing . . . . . . . . . . . . . . . 25

3.3 Synthetic testing results using plausible values . . . . . . . . . . . . 27

3.4 Synthetic testing results using sinusoid fields . . . . . . . . . . . . 28

3.5 Synthetic test for gravitational stress . . . . . . . . . . . . . . . . 29

4.1 Locations of named regions . . . . . . . . . . . . . . . . 31

4.2 Numbered regions used in tables . . . . . . . . . . . . . . . . 31

4.3 Gravitational stress results . . . . . . . . . . . . . . . . . . 33

4.4 Vertically averaged deviatoric stress results and misfits . . . . . . . . 34

4.5 Boundary stress results . . . . . . . . . . . . . . . . . 36

4.6 Effective viscosity results . . . . . . . . . . . . . . . . . . . . 37

5.1 Comparison of stress results to Lamb [2015] . . . . . . . . . . . . . . 42

5.2 Comparison of $S_{\text {Hmax }}$ directions . . . . . . . . . . . . . . 43

5.3 Log-log plot of effective viscosity against strain rate . . . . . . . . . . 46 


\section{List of Tables}

4.1 Summary of Quaternary velocity model results . . . . . . . . . . . . 31

4.2 Summary of GPS-derived velocity model results . . . . . . . . . . . . 32

B.1 Full results for Region 1 - Quaternary velocity model . . . . . . . . . 59

B.2 Full results for Region 2 - Quaternary velocity model . . . . . . . . . 60

B.3 Full results for Region 3 - Quaternary velocity model . . . . . . . . . 61

B.4 Full results for Region 4 - Quaternary velocity model . . . . . . . . . 62

B.5 Full results for Region 5 - Quaternary velocity model . . . . . . . . . 63

B.6 Full results for Region 6 - Quaternary velocity model . . . . . . . . . 64

B.7 Full results for Region 1 - GPS-derived velocity model . . . . . . . . 65

B.8 Full results for Region 2 - GPS-derived velocity model . . . . . . . . 66

B.9 Full results for Region 3 - GPS-derived velocity model . . . . . . . . 67

B.10 Full results for Region 4 - GPS-derived velocity model . . . . . . . . 68

B.11 Full results for Region 5 - GPS-derived velocity model . . . . . . . . 69

B.12 Full results for Region 6 - GPS-derived velocity model . . . . . . . . 70 


\section{Chapter 1}

\section{Introduction}

The thesis presented here is an expanded version of the manuscript, co-authored by my supervisors Simon Lamb and Martha Savage, that I have submitted to Journal of Geophysical Research. In this thesis, I explain the background and motivation for modelling the New Zealand stress field, including why more direct stress measurements are insufficient to describe the stress field within the Earth. I describe two implementations of the thin sheet approximation of the lithosphere and compare their ability to recover synthetic test stress fields. I apply one of these methods to New Zealand to calculate the deviatoric stress and effective viscosity fields for New Zealand. I compare the results I obtain for New Zealand with those obtained at other plate boundary zones and discuss the significance of the results, in particular, with reference to lithospheric rheology and earthquake stress drops.

\subsection{Motivation}

Stresses are the driving force behind plate tectonics and, therefore, earthquakes. Consequently, an understanding of the stress field could improve the understanding of earthquake hazard. While it is comparatively simple to calculate the orientation of stress, it is more difficult to calculate the magnitude of stress. The orientation of stress can be measured from earthquake focal mechanisms [e.g. Townend et al., 2012], seismic anisotropy [e.g. Savage et al., 2016], or borehole measurements [e.g. Rajabi et al., 2016]. These methods do not, however, give stress magnitudes. One 
method to estimate stress magnitudes is to calibrate them with gravitational forces. Using a knowledge of the density structure of the lithosphere, the full tensor of the stress due to gravity can be calculated. The gravitational component of the stress field can be used to calibrate the magnitude of the overall deviatoric stress [e.g. England and Molnar, 1997; Flesch et al., 2001, 2007]. A by-product of the calculation is to also provide a representation of variations in effective viscosity in the Earth, assuming a bulk viscous rheology for the lithosphere.

At shallow to moderate depths in the lithosphere, where the rocks are at lower confining pressures and temperature, rocks show a long-term elastic behaviour and deformation occurs by rupture along faults, resulting in earthquakes [Ranalli, 1995]. At the higher temperatures and confining pressures that prevail at greater depths, rocks may deform by viscous flow [Ranalli, 1995]. It has been argued that this deeper viscous flow in the mantle controls the long wavelength deformation of the lithosphere, at horizontal distances much greater than the lithospheric thickness, especially if mantle rocks are relatively strong compared to the overlying crust [England and McKenzie, 1982; England and Houseman, 1986; Houseman and England, 1986; England and Molnar, 1997; Flesch et al., 2001, 2007]. In this case, there is a direct relationship between the stress field and the rate of deformation, determined by the bulk viscosity of the lithosphere. The advantage of this approach is that it provides a tractable way to analyse both the effects of gravity and plate tectonic forces on the deformation of the lithosphere, given knowledge of the rates of deformation. In particular, for an isotropic viscous rheology, the orientation of the strain rate tensor is the same as that of the stress tensor [Ranalli, 1995].

Here, I approximate the lithosphere as a thin sheet [England and McKenzie, 1982] and calculate the vertically averaged deviatoric stress field in New Zealand. Taking advantage of the rich database of rates of deformation in the region from active faulting [Litchfield et al., 2014, GNS active fault database (www.gns.cri.nz)] and geodesy - particularly global positioning system (GPS) measurements over the past 10-20 years [Beavan et al., 2016] - I combine the gravitational stress field with possible tectonic stress fields and compare it with total stress orientations to determine the full deviatoric stress field. I use this to determine the effective viscosity field of the lithosphere. 


\subsection{Previous Work}

Stress magnitudes have been estimated by various authors, for example, in Tibet [Flesch et al., 2001], western North America [Flesch et al., 2007], and northwestern Japan [Yoshida et al., 2015]. Deviatoric stress magnitudes of 5-40 MPa were determined for these plate boundary zones. A common method of determining the deviatoric stress is to approximate the lithosphere as a thin sheet [e.g. England and Jackson, 1989; Flesch et al., 2007] and solve for vertically averaged horizontal stress. The Flesch et al. [2001] and Lamb [2015] methods for doing this are described in detail in Section 2.2. The deviatoric stress magnitudes determined by thin sheet methods are comparable to the magnitudes determined through loaded thick elastic plate calculations [e.g. Luttrell et al., 2011; Yoshida et al., 2015]. The thick elastic plate method considers the load placed on an elastic plate from above and below by topography and density variations, adding a regionally uniform horizontal boundary stress field to match the orientation of earthquake focal mechanisms [Luttrell et al., 2011]. The thick plate method is more suited to smaller, simpler areas, such as a single subduction zone, due to its assumption of a regionally uniform boundary stress field. The thin sheet methods, which do not have this assumption, can cope with more complex areas, such as the laterally variable New Zealand plate boundary zone.

Stress directions have been measured in New Zealand using earthquake focal mechanisms [e.g. Townend et al., 2012; Warren-Smith, 2016, see Fig. 5.2] and seismic anisotropy. These indicate $S_{H \max }$ directions across much of the South Island to be $\sim 110^{\circ}$, at an angle of $\sim 60^{\circ}$ to the Alpine Fault [Boese et al., 2012]. In the lower North Island, $S_{H \max }$ directions are $60^{\circ}-90^{\circ}$ for earthquakes at depths of $20-100 \mathrm{~km}$ [Townend et al., 2012]. This is in the same direction as relative plate motion, which in this region is at $259^{\circ}$ [Altamimi et al., 2012].

\subsection{Tectonic Setting}

New Zealand lies on the boundary between the obliquely converging Pacific and Australian plates (Fig. 1.1). In the North Island, the convergence of the Pacific and Australian plates is dominated by the subduction of the oceanic Pacific plate be- 
neath continental Australian plate at the Hikurangi margin at a rate of $\sim 40 \mathrm{~mm} / \mathrm{yr}$. The Hikurangi Plateau, a large igneous province that is part of the Pacific plate, is being subducted at the Hikurangi margin [summarised in Wallace et al., 2009]. Deformation is spread over an up to $250 \mathrm{~km}$ wide zone in the overlying Australian Plate [Walcott, 1978, 1984], with dextral motion accommodated by the North Island Dextral Fault Belt. The subduction interface beneath the Wellington and Wairarapa regions is strongly coupled, with a high slip-rate deficit, however, the interface further north, beneath Hawke's Bay and the Raukumara peninsula, is weakly coupled [Wallace et al., 2012]. Behind the Hikurangi margin, there has been up to $100 \mathrm{~km}$ of back-arc spreading in the Taupo Volcanic Zone and, further north, in the offshore Havre Trough [Walcott, 1978, 1984]. Several regions of repeating slow slip events have been identified along the Hikurangi margin, with recurrence intervals between 2 and 6 years and durations between 6 days and 1.5 years [summarised in Wallace and Beavan, 2010].

Subduction at the Hikurangi margin terminates beneath the northern South Island, where deformation is transferred to the continental crust and accommodated by the Marlborough Fault Zone. In the central South Island, the plate boundary is dominated by oblique continental collision with the Alpine Fault accommodating $\sim 100 \mathrm{~km}$ of shortening over the last $23 \mathrm{Ma}$ [Lamb et al., 2015] with a total dextral offset of $\sim 700 \mathrm{~km}$ [Lamb et al., 2016]. To the south, the oceanic Australian plate subducts beneath the continental Pacific plate at the Puysegur margin, off the Fiordland coast, with small amounts of shortening in Fiordland and western Otago [Walcott, 1998].

Deformation in New Zealand has been described in terms of block rotations and in terms of strain rates. Block rotations [e.g. Wallace et al., 2004, 2007] consider the deforming region to comprise a series of elastic blocks, bounded by faults, that can rotate relative to each other. Block rotations are inverted for using GPS, fault motion, and palaeomagnetic data. Calculating strain rates involves treating the deforming region as a continuously deforming elastic medium, allowing the strain rate to be calculated from the gradients of velocity. This has been done in New Zealand by numerous authors using GPS data [e.g. Beavan and Haines, 2001], fault motions [e.g. Lamb, 2015; Holt and Haines, 1995], and previously triangulation surveys [e.g. Walcott, 1984; Árnadóttir et al., 1999]. In this study, the deformation is considered 


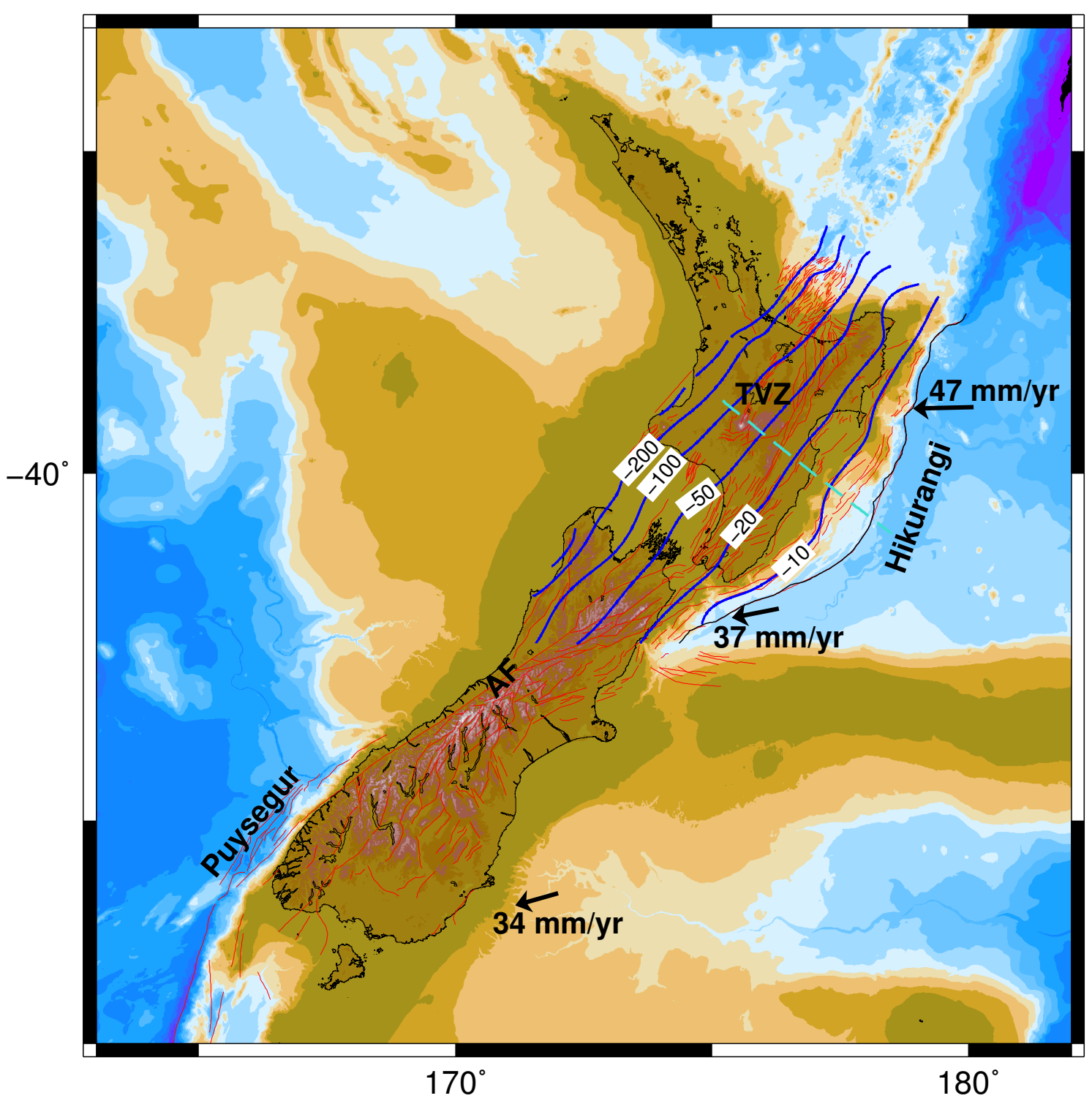

Figure 1.1: New Zealand tectonic setting, including topography and bathymetry [Mitchell et al., 2012]. Velocity vectors show the motion of the Pacific plate relative to the Australian plate [Altamimi et al., 2012]. Active faults from the New Zealand Active Fault Database [Langridge et al., 2016] are shown in red. The depth contours of the interface of the Hikurangi subduction zone [Williams et al., 2013] are shown in blue, with the Hikurangi margin shown in black. TVZ: Taupo Volcanic Zone. AF: Alpine Fault. 
in terms of strain rates to provide a continuous distribution of deformation. Consequently, any discontinuities that may exist over faults will be spread between the points on either side of the fault.

\subsection{Background Theory}

In a tectonic environment, there are three main sources of stress. Gravitational stresses are the result of the force of gravity acting on the overlying rocks in a column. These stresses act in the direction to smooth variations in topography; in the absence of other stresses, mountains would exhibit a greater tendency to collapse under their own weight due to gravitational stresses. There are two sources of boundary stresses: tectonic stresses and basal drag. Tectonic stresses result from the motions of the plates relative to each other, driven by ridge push and slab pull. Basal drag is the result of friction acting on the bottom of the lithosphere as it drags against the top of the asthenosphere.

Earthquakes result when the stresses applied to a rock exceed those the rock is able to accommodate, resulting in the fracturing of the rock or slip along an existing fault. This rock failure is governed by the Coulomb-Mohr failure criterion [Stein and Wysession, 2009]. The criterion states that if the shear stresses applied to a rock exceed the cohesive strength of the rock, subject to a normal stress, then failure will occur. Mathematically, this is expressed as

$$
\left|\tau_{s}\right|=S_{0}+\mu\left(\sigma_{n}-P_{f}\right)
$$

where $\tau_{s}$ is the shear stress, $S_{0}$ is the cohesive strength, $\mu$ is the coefficient of internal friction, $\sigma_{n}$ is the normal stress, and $P_{f}$ is the pore fluid pressure [e.g. Labuz and Zang, 2012]. The presence of faults with a low coefficient of internal friction allow failure to occur at lower shear stresses [Stein and Wysession, 2009]. The effective normal stress can also be lowered by fluid pressure, also allowing the failure to occur at lower shear stresses [Stein and Wysession, 2009]. Taken together, these factors indicate that, while stress causes earthquakes, it is not a relationship based purely on the magnitude of the stress. The relative magnitudes of the stress components, the orientation and presence of faults, and the pore fluid pressure all affect when 
failure occurs in a rock. Consequently, establishing the state of stress in a region indicates how much stress is present to drive earthquakes.

\subsubsection{Measuring Stress}

It is very difficult to measure stress directly in the context of the Earth. Consequently, several techniques have been developed to determine stress through indirect measures. Often, these measures only determine the orientation of the stress but not the magnitude and it is, thus, necessary use modelling to determine the magnitude of stress in the Earth.

The direction of the most compressive horizontal stress, $S_{H \max }$, can be determined from earthquake focal mechanisms. This involves inverting the focal mechanisms of a cluster of earthquakes using a Bayesian approach [Arnold and Townend, 2007]. This method determines the orientation of the three principal axes of stress, from which $S_{H \max }$ can be determined, and the relative magnitudes of the principal axes but not their absolute magnitudes.

The direction of $S_{\text {Hmax }}$ can also be inferred from seismic anisotropy [Savage et al., 2016]. This involves determining the fast direction of shear waves based on the arrival times of the two shear wave components. The fast direction is related to features such as the orientation of subsurface cracks. If the orientation of the cracks are determined by the regional stress field, then the fast direction gives the orientation of $S_{H \max }$ [Savage et al., 2016].

\subsubsection{Plate Boundary Stress Field}

Understanding the stress field that drives deformation in the New Zealand plateboundary zone is critical to assessing how this stress is relieved during the earthquake cycle and potentially improving predictions of when large earthquakes are likely to occur. For example, if tectonic stress magnitudes are much greater than stress drops during individual earthquakes, i.e., tectonic stress magnitudes are of the order of 100 s of $\mathrm{MPa}$, then the build up in the stress field during the seismic cycle will be difficult to detect because it will only be a small fraction of the total ambient stress. However, if tectonic stress magnitudes are comparable to earthquake stress drops, i.e. tectonic stress magnitudes are of the order of 1-10s of MPa, then significant 
changes in the stress field between earthquakes might be anticipated, which could potentially be monitored.

Here, I compare two methods of estimating tectonic or deviatoric stress, developed by Flesch et al. [2001] and Lamb [2015]. Both methods approximate the lithosphere as a thin, isotropic viscous sheet, averaging strain rates and stresses over the thickness of the sheet and solving the 2-D stress balance equations for the vertically averaged deviatoric stress; the magnitude of the deviatoric stress field is calibrated from the vertically averaged gravitational potential energy per unit volume (GPE) field. Although the two methods use the same input data of surface velocity and GPE fields for the deforming zone, they adopt different approaches to deriving the stress field (see Ch. 2). It is important to note that neither method makes any assumption about the viscous rheology of the deforming zone in terms of the relationship between viscosity and strain rate, although this relation can be potentially estimated from the stress inversion results (see Sec. 5.4).

\subsection{Definitions}

\subsubsection{Summation Convention}

This thesis uses the Einstein summation convention to simplify the presentation of equations. In this convention, an index repeated on the same side of an equation indicates summation of that index over its possible values, which are usually the directions $x, y$, and $z$ for a physical problem in three dimensions. For example, the $\sigma_{k k}$ term in equation (2.5) (representing the trace of the matrix $\sigma$ ) expands as

$$
\sigma_{k k}=\sum_{k=x, y, z}\left(\sigma_{k k}\right)=\sigma_{x x}+\sigma_{y y}+\sigma_{z z}
$$

where the index $k$ has been summed over its possible values of $x, y$, and $z$. When an index occurs once on either side of the equation, it represents a separate equation for each possible value of the index. For example, the simplified Stokes' equation (Eq. (2.4))

$$
\frac{\partial \sigma_{i j}}{\partial x_{j}}=-\rho g_{i}
$$


represents a set of three equations:

$$
\begin{aligned}
& \frac{\partial \sigma_{x x}}{\partial x}+\frac{\partial \sigma_{x y}}{\partial y}+\frac{\partial \sigma_{x z}}{\partial z}=-\rho g_{x} \\
& \frac{\partial \sigma_{y x}}{\partial x}+\frac{\partial \sigma_{y y}}{\partial y}+\frac{\partial \sigma_{y z}}{\partial z}=-\rho g_{y} \\
& \frac{\partial \sigma_{z x}}{\partial x}+\frac{\partial \sigma_{z y}}{\partial y}+\frac{\partial \sigma_{z z}}{\partial z}=-\rho g_{z}
\end{aligned}
$$

where there is one equation for each of $x, y$, and $z$ as the three possible values of $i$; $j$ has also been summed over its possible values of $x, y$, and $z$ on the left hand side.

The Kronecker delta, $\delta_{i j}$, is often used to allow equations to be easily presented using the summation convention. $\delta_{i j}$ has a value of 1 whenever $i=j$ and a value of 0 whenever $i \neq j$. For example, $\delta_{x x}=1$, because the two indices are equal, but $\delta_{x y}=0$, because the two indices are not equal.

\subsubsection{Stress Definitions}

The term 'stress' has been applied to many properties that are very similar but not exactly the same, sometimes incorrectly [Engelder, 1994]. In order to avoid confusion, it is desirable to define what is meant by the term 'stress' and its derivative terms. Stress is the force per unit area exerted on material inside a surface by material outside a surface. For each of the three face directions $(x, y$, and $z)$ of the surface, there are three directions of forces that can act on that surface [Stein and Wysession, 2009]. This results in nine components of total stress; these are also referred to as tractions. Of the nine components, only six are independent, due to symmetry of the $x y / y x, x z / z x$, and $y z / z y$ components. Total stress refers to these components when considered together. These are represented mathematically by the total stress tensor

$$
\sigma=\left(\begin{array}{lll}
\sigma_{x x} & \sigma_{x y} & \sigma_{x z} \\
\sigma_{x y} & \sigma_{y y} & \sigma_{y z} \\
\sigma_{x z} & \sigma_{y z} & \sigma_{z z}
\end{array}\right)
$$

where $\sigma_{x x}, \sigma_{x y}$, etc., are the components of total stress. Shear stress refers to the $\sigma_{x y}$, $\sigma_{x z}$, and $\sigma_{y z}$ components, where the force acts parallel to the surface, resulting in a shearing force. This is contrasted to the normal stress components $\sigma_{x x}, \sigma_{y y}$, and $\sigma_{z z}$, 
where the force acts perpendicular to the surface, acting to extend or compress the material. In this study, compression is taken as negative and extension as positive, as is conventional for seismological studies [Stein and Wysession, 2009], although the opposite convention with compression positive is sometimes used in other disciplines.

The relative sizes of the stress components depends on the orientation of the coordinate system they are measured in. For any given stress tensor, there is a unique rotation of the coordinate system such that the shear stress components are zero and only the normal stress components are non-zero [Stein and Wysession, 2009]. When the shear stresses are zero, the normal stresses represent the principal stresses, often numbered $S_{1}, S_{2}$, and $S_{3}$ from most compressive to most extensional, and their directions represent the principal axes of stress. Mathematically, the principal axes of stress are the eigenvectors of the stress matrix and the principal stresses are the associated eigenvalues. The principal axes and stresses represent a convenient way to visualise the stress tensor and are used in this thesis for the presentation of stresses.

Deviatoric stress is the total stress but with the pressure (average normal stress) removed from the normal stress components (presented using the summation convention described in Subsection 1.5.1):

$$
\tau_{i j}=\sigma_{i j}-\delta_{i j} P=\sigma_{i j}-\frac{1}{3} \delta_{i j} \sigma_{k k}
$$

where $\tau_{i j}$ is the $i j^{\text {th }}$ component of deviatoric stress and $P$ is the pressure, equal to one-third of the trace of the total stress tensor, $\sigma_{k k} / 3$. The presence of the Kronecker delta, $\delta_{i j}$, indicates that the pressure term is only removed from the normal stress components but not from the shear stress components. The deviatoric shear stress components are identical to their total stress equivalents. Because the same pressure term is removed from the normal stress components, the principal axes of deviatoric and total stress align (but with a constant difference between the size of the principal deviatoric and total stresses). This allows the orientation of total stress and deviatoric stress to be compared directly.

Because the full description of the stress tensor requires six numbers, it can be more convenient to describe the stress in terms of a single indicative number. In this study, stress magnitudes are given as the square-root of the second invariant of 
the stress tensor, that is, for deviatoric stress, the square-root of

$$
\tau_{I I}=\tau_{i j} \tau_{j i}
$$

Stress directions are sometimes given in terms of $S_{\text {Hmax }}$ : this is the direction of maximum horizontal compressive stress. If one of the principal stresses is vertical, then $S_{H \max }$ is in the direction of one of the other two principal stresses, however, this is not generally the case [e.g. Townend et al., 2012].

Differential stress is the difference between most compressive principal stress $S_{1}$ and the most extensional principal stress $S_{3}$, i.e., differential stress equals $S_{1}-S_{3}$. 


\section{Chapter 2}

\section{Method}

I compare two different methods to determine the deviatoric stress field in New Zealand. Both methods, developed by Flesch et al. [2001] and Lamb [2015], use a thin sheet approximation of the lithosphere to solve the 2-D stress balance equations for the vertically averaged deviatoric stress field. They use the variations in gravitational potential energy per unit volume (GPE) to calibrate the magnitude of the deviatoric stress and associate the orientation of the deviatoric stress to the orientation of the strain rate through an isotropic viscosity. I also use two surface velocity models for the New Zealand region, one based on Quaternary fault motions [Lamb, 2015] and one based on GPS velocities.

\subsection{Data}

Two velocity models are used in this study: one based on Quaternary fault motions [Lamb, 2015] and one derived from GPS velocities. For later calculations, it is more convenient to re-express the horizontal velocities $v_{x}$ and $v_{y}$ in terms of the components of the strain rate tensor $\dot{\varepsilon}$ as

$$
\dot{\varepsilon}_{i j}=\frac{1}{2}\left(\frac{\partial v_{i}}{\partial x_{j}}+\frac{\partial v_{j}}{\partial x_{i}}\right)
$$

for the $i j^{t h}$ component of the strain rate $\dot{\varepsilon}_{i j}$, where $u_{i}$ is the component of velocity in the $x_{i}$ direction. The strain rate components were calculated on a spherical grid 
by finite differences at $0.5^{\circ}$ spacing longitudinally and latitudinally.

\subsubsection{Quaternary Velocity Field}

The Quaternary velocity model represents deformation during the Quaternary, averaging fault motions over several earthquake cycles and recording strain accumulated over tens to hundreds of thousands of years. The model used here is the model from Lamb [2015], calculated using the method of Lamb [2000]. This method splits the region into a series of triangles whose vertices are aligned with major plate boundary zone features. Knowledge of the velocities at the vertices of a triangle uniquely determines the average strain rates within that triangle, mainly based on fault slip rate data or patterns of active faulting [Lamb, 2015]. Known velocities or average strain rates for one triangle place constraints on the velocities and average strain rates in the neighbouring triangle. An added constraint is the instantaneous relative motion between the Australian and Pacific plates, based on GPS data [Beavan et al., 2002]. With enough velocity and strain rate estimates, the velocities and strain rates for the network of triangles can be solved as a set of linear equations.

\subsubsection{GPS-derived Velocity Field}

The GPS-derived model represents the very short term contemporary deformation, based on repeated dense campaign measurements on a timescale of years to a few decades [Beavan et al., 2016]. Deformation on this timescale in a plate boundary zone such as New Zealand is mainly elastic and mainly represents strain/stress accumulation that will ultimately be released in earthquakes [Lamb and Smith, 2013]. The GPS-derived velocities are well constrained for onshore New Zealand due to continuous GPS sites and repeated dense campaign GPS [Beavan et al., 2016]. The GPS-derived velocity field is specified for the stable Australian and Pacific plates based on the relative plate motion between the two plates. The relative plate motion is set using the Euler pole of rotation [Altamimi et al., 2012], treating those parts of the plates as rigid. In order to extrapolate the velocity field offshore, where there are no GPS measurements, and to interpolate the measurements of Beavan et al. [2016], bicubic splines are fitted to the data [Beavan and Haines, 2001]. The interpolation is done using GMT surface, which uses the method of Smith and Wessel [1990]. Strain 


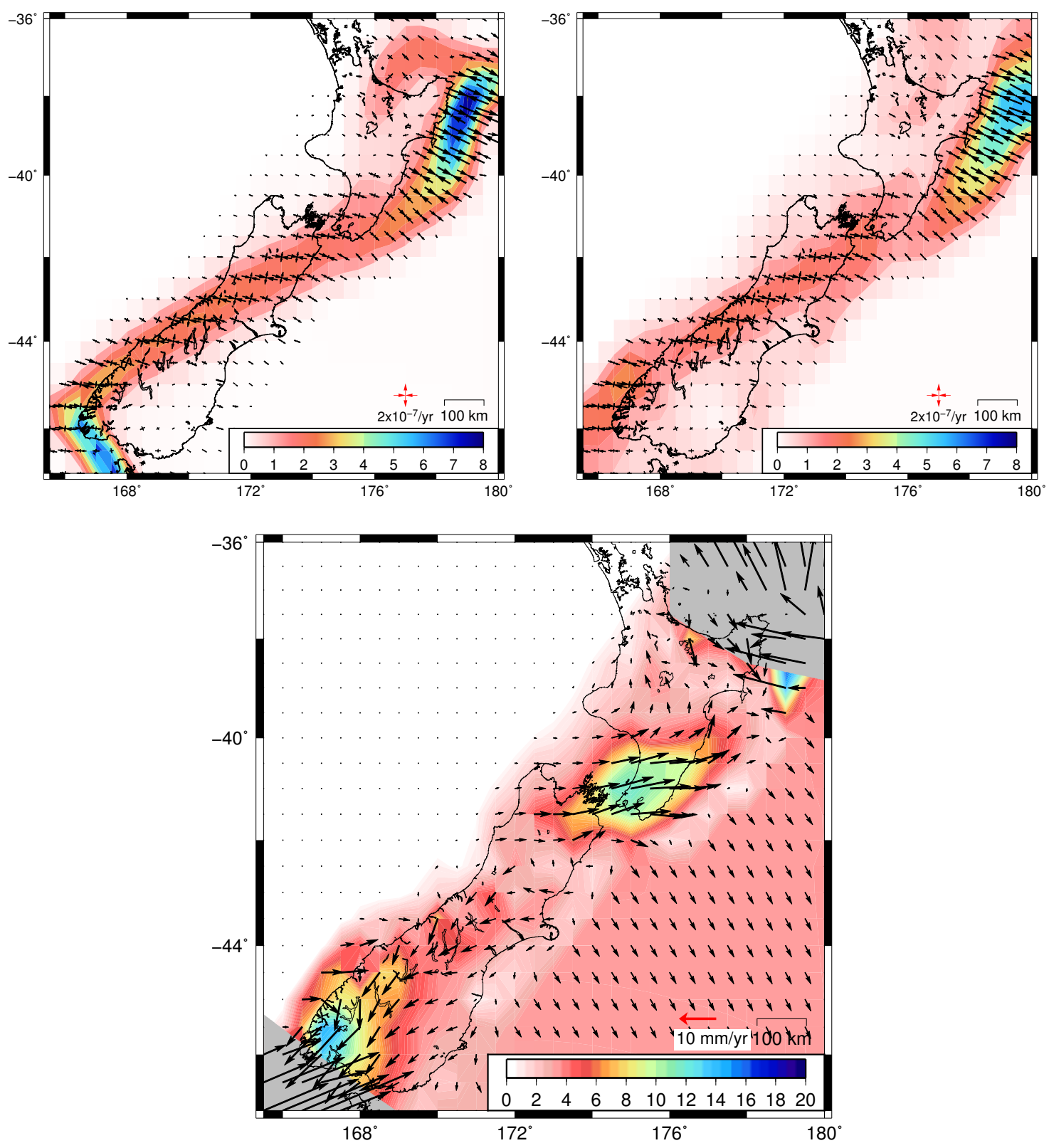

Figure 2.1: Comparison of the two velocity models used in this study. Top Left: Strain rates based on Quaternary velocities [Lamb, 2015]. The arrows indicate the strain rate principal axes, with compression being inwards and extension outwards. The colours indicate the strain rate magnitudes in $\times 10^{-7} / \mathrm{yr}$. Top Right: Strain rates based on GPS data. Bottom: The difference in velocities (arrows) between the two velocity models, taken as the Quaternary fault motion model minus the GPS-derived model. The colours indicate the magnitude of the velocity difference in $\mathrm{mm} / \mathrm{yr}$. The grey shaded regions indicate regions that are masked in later figures. 
rates are calculated as the spatial derivatives of velocity using equation (2.1). The strain rates are calculated on a spherical grid by finite differences at $0.5^{\circ}$ spacing longitudinally and latitudinally.

The two models (Fig. 2.1) are generally similar. The most notable difference between the two models occurs in the southern North Island, where the GPS-derived velocities show greater westerly velocities than the Quaternary velocities, most likely due to elastic strain accumulation in this region as a result of locking on the underlying plate interface [Darby and Beavan, 2001; Lamb and Smith, 2013]. Similarly, in the southern South Island, the Quaternary velocity model shows greater southwesterly velocities than the GPS-derived velocity model, presumably for similar reasons. The deformation, shown by the strain rate, extends over a greater width in the GPSderived velocity model than the Quaternary velocities. This deformation, however, has a smaller magnitude in the GPS-derived model, resulting in the same total deformation across the plate boundary zone. The difference in between the models to the southeast of New Zealand, on the assumed stable part of the Pacific plate, is due to the GPS-derived model using the more recent Altamimi et al. [2012] relative plate motions, whereas, the Quaternary velocity model uses [Beavan et al., 2002] relative plate motions. Both models are poorly constrained to the northwest of the North Island and to the south of the South Island as both models lack data in these regions.

\subsubsection{Gravitational Potential Energy per Unit Volume}

For a column of rock, gravitational potential energy per unit volume (GPE) is equivalent to the negative of the vertical normal stress at depth $z$ in the column [England and Houseman, 1986]:

$$
\sigma_{z z}=-\int_{0}^{z} g \rho\left(z^{\prime}\right) d z^{\prime}
$$

averaged over the column. That is, GPE, $\Gamma$, is given by

$$
\Gamma=-\frac{1}{L} \int_{0}^{L} \sigma_{z z}(z) d z=\frac{1}{L} \int_{0}^{L} \int_{0}^{z} g \rho\left(z^{\prime}\right) d z^{\prime} d z
$$


for a column of height $L$. In terms of the thin sheet model, this depth-averaged value defines the average vertical stress in the thin sheet, used in the stress balance equations (see below), with $L$ being the thickness of the thin sheet. The GPE field used in this study is from Lamb [2015] and was calculated using typical crustal and mantle densities based on a crustal structure produced from receiver function, seismic reflection, and gravity data (see Lamb 2015 for details). Lamb [2015] actually calculated contrasts in GPE relative to a datum, however, this is not important in the following analysis because the key parameters in the stress balance equations (Eq. (2.7)) are the gradients of GPE, rather than their absolute values. Variations in GPE mainly reflect the topography of the region ( $\sim 70 \%$ contribution) with a lesser contribution ( $\sim 30 \%)$ from variations in crustal thickness. For this reason, average stresses are relatively insensitive to the thickness of the deforming zone (see Sec. 5.3).

\subsection{Stress Models}

\subsubsection{Stress Balance Equation}

In order to determine the stress field, it is necessary to relate it to physical quantities that can be measured or estimated. In this case, I link the deviatoric stress to strain rate and vertically averaged gravitational potential energy per unit volume (GPE) using the viscous thin sheet approximation of the lithosphere of England and Molnar [1997]. This assumes that the lithosphere behaves as a continuously deforming solid, i.e., it is viscous, and its deformation is governed by Stokes' equation:

$$
\frac{\partial \sigma_{i j}}{\partial x_{j}}=-\rho g_{i}
$$

where $\sigma_{i j}$ is the $i j^{t h}$ component of the total stress tensor, $\rho$ is the density and $g_{i}$ is the component of the gravitational acceleration in the $x_{i}$ direction. Equation (2.4) is a volumetric force balance equation, relating the force of gravity per unit volume to the gradients of stress. This assumes that the accelerations of the plates are negligible.

I assume that the shear tractions acting on the base of the lithosphere are small, 
as has been done in other studies [e.g. England and Jackson, 1989; England and Molnar, 1997; Flesch et al., 2001]. This allows the vertical shear stress, $\sigma_{x z}$ and $\sigma_{y z}$, components of the total stress tensor to be neglected. The vertical component of the total stress $\sigma_{z z}$ at a depth $z$ is now given by equation (2.2), allowing $\sigma_{z z}$ at any depth to be determined from topography and the density structure of the lithosphere above it.

As has also been done by other studies [e.g. England and Molnar, 1997; Flesch et al., 2001; Lamb, 2015], I vertically average equation (2.4). This reduces the problem from a full three dimensional problem to one of a thin sheet, solving for vertically averaged horizontal stresses. It is also more convenient to express the stress balance equation in terms of deviatoric stress $\tau$, defined as

$$
\tau_{i j}=\sigma_{i j}-\frac{1}{3} \delta_{i j} \sigma_{k k}
$$

for $i j^{t h}$ component of $\tau$. This is equivalent to removing the average, given by onethird of the trace of the total stress tensor, $\sigma_{k k} / 3$, from the normal components of the total stress. The shear components remain unchanged. The orientations of the principal stresses of total stress and deviatoric stress are the same. Expressing equation (2.4) in terms of the deviatoric stress allows the use of the relationship

$$
\tau_{x x}+\tau_{y y}+\tau_{z z}=0
$$

which emerges as a consequence of the definition of deviatoric stress in equation (2.5). This allows $\tau_{z z}$ to be re-expressed in terms of $\tau_{x x}$ and $\tau_{y y}$, simplifying the stress balance equation.

Combining equations (2.4)-(2.6) to express the stress balance equation in terms of the vertically averaged deviatoric stress results in

$$
\begin{array}{r}
2 \frac{\partial \tau_{x x}}{\partial x}+\frac{\partial \tau_{y y}}{\partial x}+\frac{\partial \tau_{x y}}{\partial y}=\frac{\partial \Gamma}{\partial x} \\
2 \frac{\partial \tau_{y y}}{\partial y}+\frac{\partial \tau_{x x}}{\partial y}+\frac{\partial \tau_{x y}}{\partial x}=\frac{\partial \Gamma}{\partial y}
\end{array}
$$

where $\Gamma$ is the vertically averaged gravitational potential energy per unit volume (GPE) given by equation (2.3). Like $\sigma_{z z}, \Gamma$ can be determined from a knowledge 
of the density structure of the lithosphere. This means that equation (2.7) relates the horizontal gradients of the three horizontal components of deviatoric stress to the two horizontal gradients of a quantity which can be determined, namely GPE. Equation (2.7) corresponds to two equations with three unknowns; a further constraint is required in order to be able to solve it. I test two methods of solving equation (2.7). The Flesch et al. [2001] method (Subsec. 2.2.2; referred to hereafter as the stress method) solves first for deviatoric stress due to variations in density by applying a stress minimisation constraint and then solves for the full deviatoric stress by applying the constraint that the strain rate and stress fields are, as close as possible, related by an isotropic effective viscosity. The Lamb [2015] method (Subsec. 2.2.3; referred to hereafter as the strain rate method) applies an isotropic viscosity constraint directly to equation (2.7), restating the equation in terms of strain rate and viscosity. It then solves for the isotropic viscosity, which can be converted to a stress when combined with the strain rate.

An isotropic viscosity is one that is the same in all directions and can be represented mathematically by a single scalar value, however, it can vary spatially. This is equivalent to saying that the orientation and style of the stress and strain rate tensors are the same. Both methods use the orientation of the strain rate to determine the orientation of the stress. Because the calculated deviatoric stress is vertically averaged, using one strain rate for the entire column is to assume that the strain rate does not vary with depth and that the strain rate at the surface applies for all depths over the lithosphere. This assumption is necessary because we do not have information on the strain rate at depth; we only have information on the strain rate at the surface.

\subsubsection{Stress Method}

Flesch et al. [2001] use a two step method to solve equation (2.7) for the deviatoric stress field. They solve first for the gravitational deviatoric stress due to variations in topography and in the density structure of the lithosphere, seeking the minimum stress field solution to equation (2.7). To this, they add boundary stresses resulting from tectonic stresses, seeking a stress field with orientations that most closely match the orientations of the strain rate field; this is equivalent to seeking an isotropic 
viscosity.

To solve for the gravitational stress field, Flesch et al. [2001] seek the solution to equation (2.7) with the minimum total deviatoric stress magnitude. They seek to minimise the second invariant of the deviatoric stress tensor,

$$
\tau_{I I}=2 \tau_{x x}^{2}+2 \tau_{x x} \tau_{y y}+2 \tau_{y y}^{2}+2 \tau_{x y}^{2}
$$

integrated over the region of interest. Seeking the stress field with the minimum magnitude, given by the (square-root of the) second invariant of the deviatoric stress, is to seek the stress due only to body forces from within the region and to exclude stresses due to boundary forces from outside the region (which are considered later). In this way, the minimum magnitude stress field is the stress field due just to gravitational forces within the region of interest.

Flesch et al. [2001] show that minimisation of equation (2.8) whilst solving equation (2.7) occurs when

$$
\tau_{i j}=\frac{1}{2}\left(\frac{\partial \lambda_{i}}{\partial x_{j}}+\frac{\partial \lambda_{j}}{\partial x_{i}}\right),
$$

where $\lambda_{i}$ is a Lagrange multiplier, for all points inside the region, and $\lambda_{i}=0$ for all points on the boundary. This allows the three horizontal components of deviatoric stress to be expressed in terms of two Lagrange multipliers. Substituting equation (2.9) into equation (2.7) yields

$$
\begin{aligned}
& 2 \frac{\partial^{2} \lambda_{x}}{\partial x^{2}}+\frac{3}{2} \frac{\partial^{2} \lambda_{y}}{\partial x \partial y}+\frac{1}{2} \frac{\partial^{2} \lambda_{x}}{\partial y^{2}}=\frac{\partial \Gamma}{\partial x} \\
& 2 \frac{\partial^{2} \lambda_{y}}{\partial y^{2}}+\frac{3}{2} \frac{\partial^{2} \lambda_{x}}{\partial y \partial x}+\frac{1}{2} \frac{\partial^{2} \lambda_{y}}{\partial x^{2}}=\frac{\partial \Gamma}{\partial y}
\end{aligned}
$$

This is a set of two partial differential equations with two unknowns that can be solved using numerical methods. The gravitational deviatoric stress can then be recovered from $\left(\lambda_{x}, \lambda_{y}\right)$ using equation $(2.9)$.

The full deviatoric stress field is the sum of the gravitational stresses solved for in equation (2.10) and boundary stresses. Flesch et al. [2007] represent the boundary stress as a linear combination of basis functions. These basis functions are solutions to the homogeneous stress balance equations; they are functions that solve 
equation (2.7) when $\Gamma$ is set to 0 . Because equation (2.7) is linear in stress, any linear combination of solutions to the homogeneous equation can be added to a particular solution of equation (2.7) and the result will be a solution to equation (2.7).

To create the basis functions, Flesch et al. [2007] set $\left(\lambda_{x}, \lambda_{y}\right)=\boldsymbol{\Omega} \times \mathbf{r}$ for a segment of the boundary, where $\mathbf{r}$ is the position vector of the point on the boundary and $\Omega$ is a unit vector in the $x, y$, or $z$ direction. $\left(\lambda_{x}, \lambda_{y}\right)$ is set to 0 for the remainder of the boundary and equation (2.10) is solved for $\left(\lambda_{x}, \lambda_{y}\right)$ with $\Gamma=0$. The $\left(\lambda_{x}, \lambda_{y}\right)$ values are converted into their equivalent values of deviatoric stress using equation (2.9) to create a set of basis functions that represent possible boundary stresses.

Another constraint is required to determine which combination of basis functions, when combined with the gravitational stress field, represents the physical stress field. Flesch et al. [2001] seek a stress field that most closely matches the style and orientation of the strain rate field. This is equivalent to seeking an isotropic effective viscosity.

Flesch et al. [2001] seek to match the orientations of the stress field and strain rate field by minimising the objective function

$$
\int_{S}(E T-e \tau) d S
$$

where

$$
\begin{aligned}
E & =\sqrt{2 \dot{\varepsilon}_{x x}^{2}+2 \dot{\varepsilon}_{x x} \dot{\varepsilon}_{y y}+2 \dot{\varepsilon}_{y y}^{2}+2 \dot{\varepsilon}_{x y}^{2}}, \\
T & =\sqrt{2 \tau_{x x}^{2}+2 \tau_{x x} \tau_{y y}+2 \tau_{y y}^{2}+2 \tau_{x y}^{2}}, \\
e \tau & =\sqrt{2 \dot{\varepsilon}_{x x} \tau_{x x}+\dot{\varepsilon}_{x x} \tau_{y y}+\dot{\varepsilon}_{y y} \tau_{x x}+2 \dot{\varepsilon}_{y y} \tau_{y y}+2 \dot{\varepsilon}_{x y} \tau_{x y}},
\end{aligned}
$$

and the objective function is integrated over the region of interest. $E$ and $T$ are the square-root of the second invariant of the strain rate and deviatoric stress tensors, respectively, referred to here as the strain rate and deviatoric stress magnitudes. The objective function at a particular point is zero when the deviatoric stress tensor is a scalar multiple of the strain rate tensor, with that scalar multiple being the effective viscosity. The effective viscosity $\eta$ of a point can be determined as $\eta=T / E$, i.e., the magnitude of the deviatoric stress divided by the magnitude of the strain rate.

How well the modelled stress fits the strain rate can be quantified using the 
misfit. Flesch et al. [2007] define the misfit for each point as

$$
M=\frac{1}{2}\left(1-\frac{e \tau}{E T}\right)
$$

The misfit has a minimum value of 0 when the modelled stress is a scalar multiple of the strain rate and a maximum value of 1 when the stress and strain rate orientations are maximally misaligned.

\subsubsection{Strain Rate Method}

Lamb [2015] solves equation (2.7) by assuming an isotropic viscosity, relating the components of deviatoric stress and strain rate by

$$
\tau_{i j}=\eta \dot{\varepsilon}_{i j}
$$

where $\eta$ is the isotropic effective viscosity that is the same for all components of the deviatoric stress and strain rate tensors for a given location. Equation (2.14) is substituted into equation (2.7) and allowing $\eta$ to vary spatially results in

$$
\begin{aligned}
& \eta\left(2 \frac{\partial \dot{\varepsilon}_{x x}}{\partial x}+\frac{\partial \dot{\varepsilon}_{y y}}{\partial x}+\frac{\partial \dot{\varepsilon}_{x y}}{\partial y}\right)+\frac{\partial \eta}{\partial x}\left(2 \dot{\varepsilon}_{x x}+\dot{\varepsilon}_{y y}\right)+\frac{\partial \eta}{\partial y} \dot{\varepsilon}_{x y}=\frac{\partial \Gamma}{\partial x} \\
& \eta\left(2 \frac{\partial \dot{\varepsilon}_{y y}}{\partial y}+\frac{\partial \dot{\varepsilon}_{x x}}{\partial y}+\frac{\partial \dot{\varepsilon}_{x y}}{\partial x}\right)+\frac{\partial \eta}{\partial y}\left(2 \dot{\varepsilon}_{y y}+\dot{\varepsilon}_{x x}\right)+\frac{\partial \eta}{\partial x} \dot{\varepsilon}_{x y}=\frac{\partial \Gamma}{\partial y}
\end{aligned}
$$

As the strain rate $\dot{\varepsilon}$ and the GPE $\Gamma$ are known, equation (2.15) can be solved as a pair of simultaneous differential equations for $\eta$. Because equation (2.15) is linear in terms of $\dot{\varepsilon}$, any linear combination of solutions to the homogeneous equation (where $\Gamma=0)$ can be added to a particular solution, with the result also being a solution to equation (2.15). If the only solution to the homogeneous equation is the trivial solution (i.e., $\eta=0$ everywhere), then the only solution to equation (2.15) is the particular solution. If, however, there are non-trivial solutions to the homogeneous equation, then it is necessary to add a multiple of the solution to the homogeneous equation to the particular solution. Lamb [2015] determines the multiple of the homogeneous solution required to add to the particular solution by using prior knowledge, such as an assumed stress at a particular point or an assumed average 
viscosity. The deviatoric stress can be recovered from $\eta$ and the strain rate using equation (2.14).

It is important to emphasise that no assumption is made in either the stress or strain rate methods about the relation between strain rate and viscosity (i.e., whether the viscous rheology is Newtonian or some power law). However, once the effective viscosity is calculated, this relation can be potentially estimated from the observed strain rate field and is done so in Section 5.4. 


\section{Chapter 3}

\section{Synthetic Testing}

\subsection{Testing Method}

Synthetic testing is performed to test the ability of the Flesch et al. [2001] stress method and the Lamb [2015] strain rate method to recover a known input stress or viscosity field. Synthetic viscosity and velocity fields are chosen (Fig. 3.1)and the corresponding GPE fields that satisfy equation (2.7) are calculated (Fig. 3.2). Normally-distributed noise is added to the velocity field. The standard deviations of the noise used, in terms of the range of the velocity, are $0 \%$ (i.e. no noise), $1 \%$, $5 \%$, and $20 \%$. The velocity field with added noise and the GPE field are used as inputs to the two methods and the output viscosity field is compared to the initial synthetic viscosity. Two synthetic viscosity and velocity field pairs are tested, one based on sinusoids and one with values plausible for the New Zealand region.

The plausible-values synthetic model (Fig. 3.1, left) consists of a central low viscosity zone, representing a plate boundary zone, with higher viscosity on either side, representing stable plates. The central zone has a viscosity of $10^{21} \mathrm{~Pa} \mathrm{~s}$, smoothly increasing to a viscosity of $10^{22} \mathrm{~Pa}$ s on either side [cf. Ghosh and Holt, 2012]. The velocity for the high viscosity sides was chosen to represent the relative plate motions between the Australian and Pacific plates [Altamimi et al., 2012], with the velocity smoothly varying across the low viscosity zone. The velocities vary between 0-50 mm/yr. The corresponding GPE field that satisfies equation (2.7) has a range of $40 \mathrm{MPa}$. This model aims to test the methods' ability to recover the 

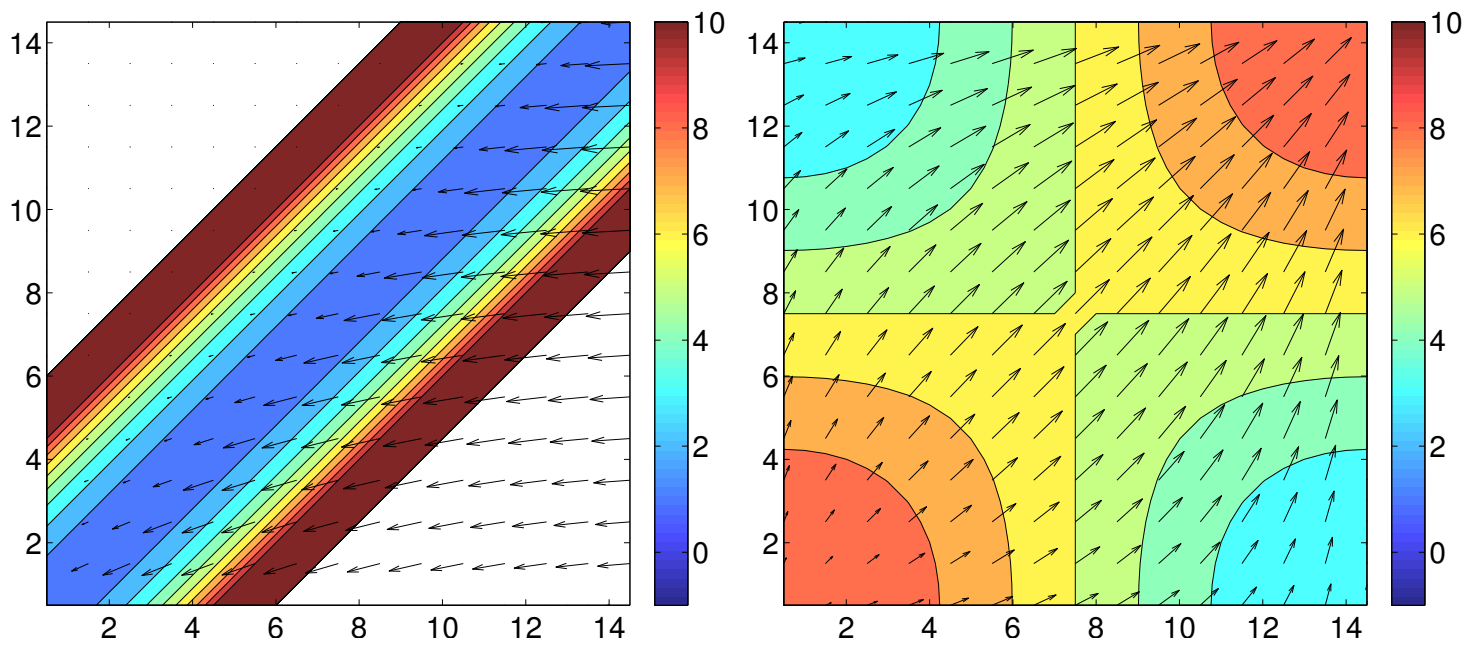

Figure 3.1: Input viscosity and velocity fields used to test the ability of the Flesch et al. [2001] and Lamb [2015] methods to recover an input viscosity field. The axis units are $100 \mathrm{~km}$. The colour bar shows the viscosities in $10^{21} \mathrm{~Pa}$ s. Velocities are shown as arrows. Left: Fields based on plausible values; largest velocity is $50 \mathrm{~mm} / \mathrm{yr}$. Right: Fields based on sinusoids; largest velocity is $70 \mathrm{~mm} / \mathrm{yr}$.

type of stress and viscosity fields that are thought to be present in the New Zealand region.

The sinusoid model (Fig. 3.1, right) consists of a viscosity field formed from the product of two sinusoids with an added constant, ranging between $3-9 \times 10^{21} \mathrm{~Pa}$ s. Each component of the velocity field is similarly formed from the product of two sinusoids, with the velocity ranging between $0-70 \mathrm{~mm} / \mathrm{yr}$. The corresponding GPE field that satisfies equation (2.7) has a range of $130 \mathrm{MPa}$. This model aims to test the methods' ability to recover stress and viscosity fields with large variations.

\subsection{Testing Results}

The strain rate method is able to recover the input viscosity fields (Figs. $3.3 \&$ \&.4) when no noise had been added to the velocity. However, its ability to recover the input viscosity rapidly decreases as increasing levels of noise are added to the velocity. This rapid decrease in its ability to recover the viscosity field is due to the strain rate method involving the gradients of strain rate in equation (2.15) or, equivalently, the second derivatives of velocity. As differentiation amplifies the 

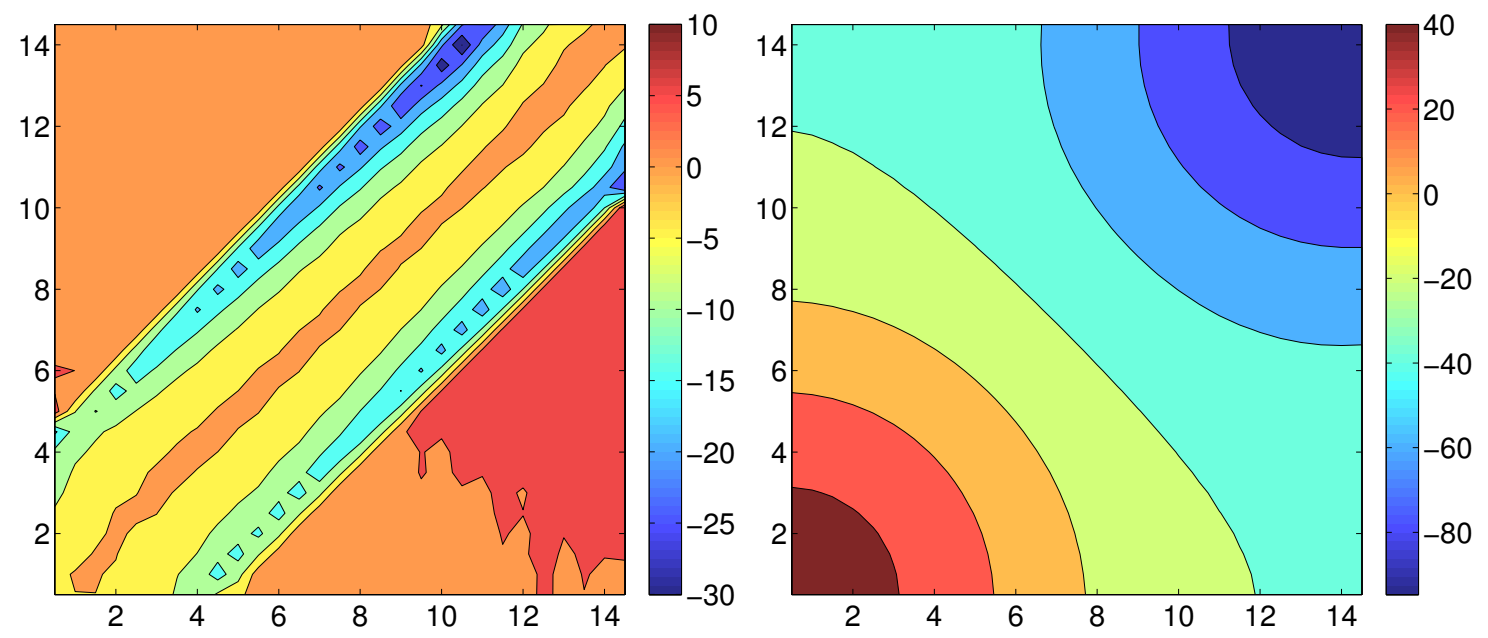

Figure 3.2: GPE fields used in synthetic testing, calculated from the velocity and viscosity fields shown in Figure 3.1. The axis units are $100 \mathrm{~km}$. The colour bar shows GPE in MPa relative to an arbitrary point. Left and right correspond to GPE fields calculated from Figure 3.1 left and right, respectively.

higher frequency noise, the strain rate method's reliance on the second derivative of velocity makes it susceptible to noise. Increasing the noise tends to have the effect of reducing the viscosity determined by the strain rate method unless solutions to the homogeneous version of equation (2.15) are added to the initially outputted viscosity.

The stress method is able to recover the magnitude and general style of the input viscosity fields (Figs. 3.3 \& 3.4) when no noise has been added, however, it does so with noticeable 'blurring'. This is because the stress method describes the stress field as a combination of stress basis functions, which can only represent smooth boundary stress fields. Furthermore, because the boundary stress is represented as the linear combination of a series of basis functions, unless the boundary stress is an exact linear combination of the basis functions, the stress method is unable to exactly represent the boundary stress. Consequently, the stress method finds the linear combination of basis functions that most closely matches the boundary stress but as this is not exactly the boundary stress, it will not exactly recover the input viscosity. The stress method is better able to handle higher levels of noise added to the velocity than the strain rate method as the stress method only involves the strain rate itself, in equation (2.11), and does not involve the derivative of strain 
rate. Additionally, because the boundary stress field is represented by smooth basis functions, only smooth boundary stress fields can be represented. This results in a smoothing of the calculated stress field, reducing the effect of noise.

The uncertainties in the Quaternary field are typically $<1.5 \mathrm{~mm} / \mathrm{yr}$ [Lamb, 2015], corresponding to $<\sim 3 \%$ of the range of the velocity field. Furthermore, correlations between neighbouring grid points would reduce the uncertainty in the gradients of velocity (strain rates), which were not included in the synthetic testing. This indicates that the methods, especially the stress method, should be able to recover the essential features of the deviatoric stress and effective viscosity fields in New Zealand using the available data of velocities and GPE, albeit with some 'blurring' of details.

\subsection{Recovering Gravitational Stress Field}

A further test is performed to test the stress method's ability to calculate gravitational stresses from variations in GPE. For this, a cone with a constant density structure is chosen because it has a simple, analytical solution. The horizontal gravitational stresses around a cone are expected to have one principal axis of stress radial and the other principal axis circumferential. This is equivalent to that if GPE varies only in the $x$-direction, i.e. $\Gamma=\Gamma(x)$, then the deviatoric stresses will be

$$
\begin{aligned}
\tau_{x x} & =\frac{1}{2} \Gamma(x)+\text { constant } \\
\tau_{y y} & =0 \\
\tau_{x y} & =0
\end{aligned}
$$

These can be substituted into equation (2.10) to prove that they represent the minimum magnitude solution to equation (2.7). An equivalent form exists for the case of the radial symmetry that exists around a cone, with both the circumferential and shear stresses being zero and only the radial stress being non-zero. The form of the deviatoric stress is unsurprising. When there is no variation in the circumferential direction (i.e., around the cone), there is no source of force in that direction. The variation and, therefore, the only force is in the radial. Consequentially, the radial 


\section{Strain Rate Method}
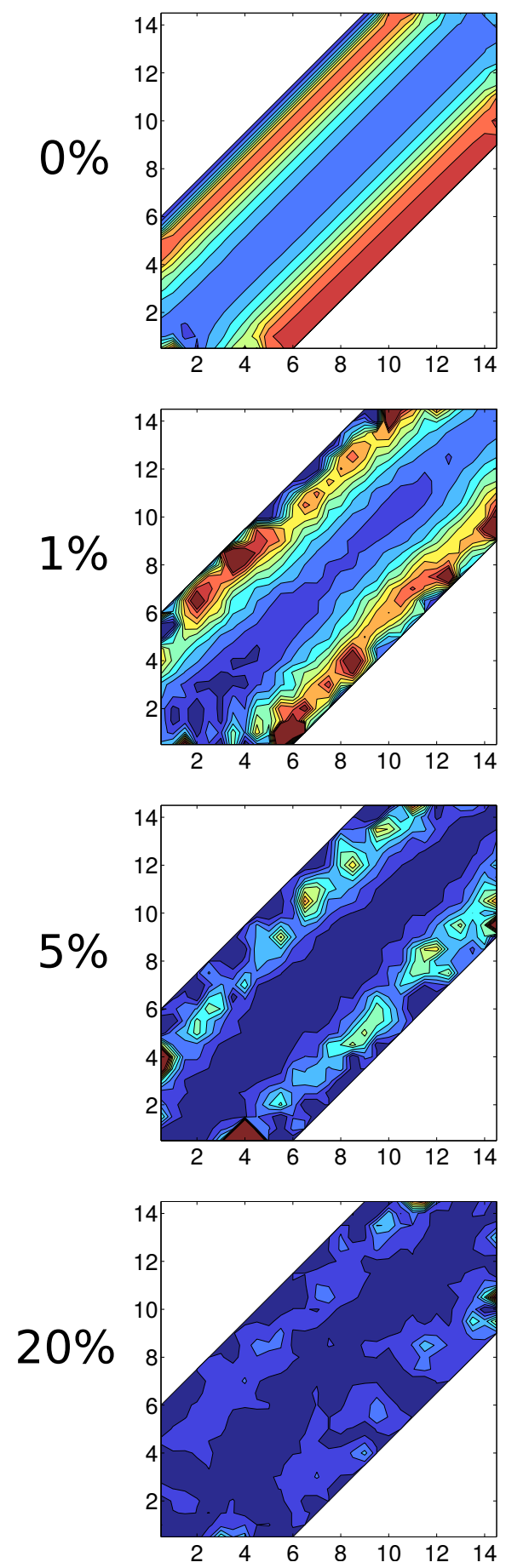

Stress Method
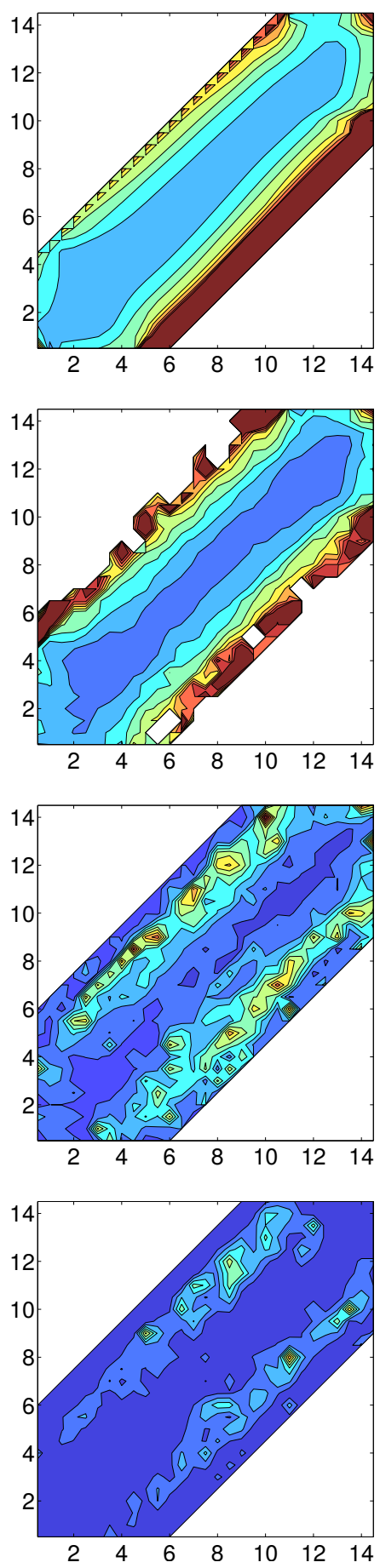

Figure 3.3: Viscosity field recovered using the Lamb [2015] strain rate (first column) and Flesch et al. [2001] stress (second column) methods. The input viscosity field is the plausible-values field shown in figure 3.1 (left). The rows show increasing noise levels. First row: No noise. Second row: Noise approximately $1 \%$ of velocity range. Third row: Noise approximately $5 \%$ of velocity range. Fourth row: Noise approximately $20 \%$ of velocity range. 
Strain Rate Method
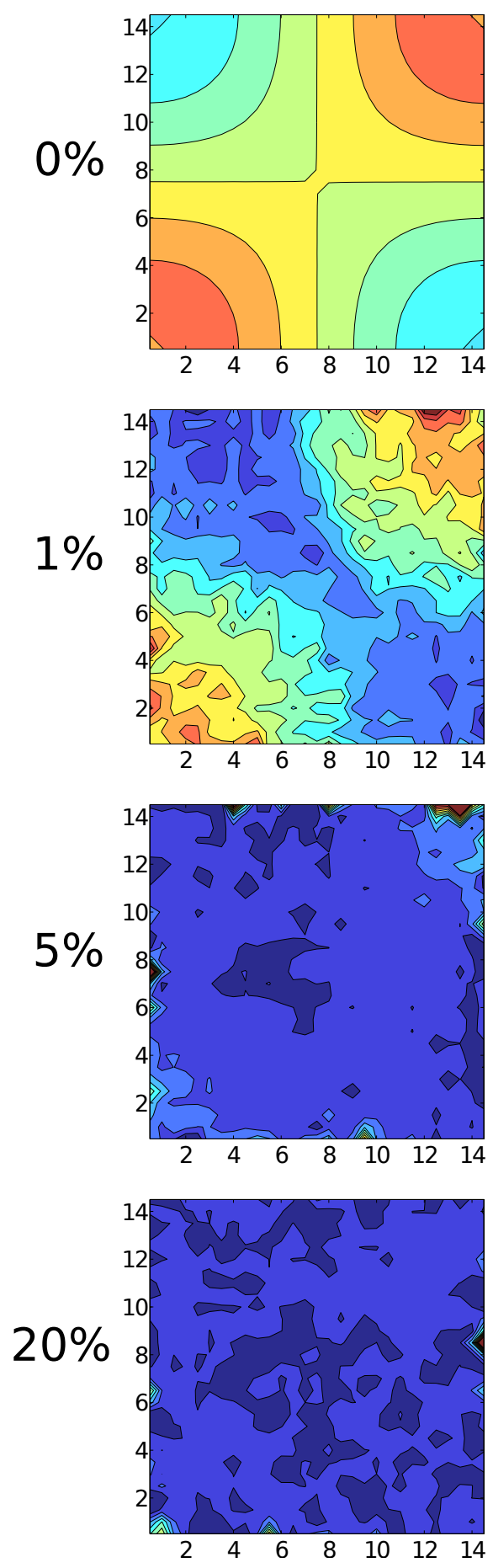

Stress Method
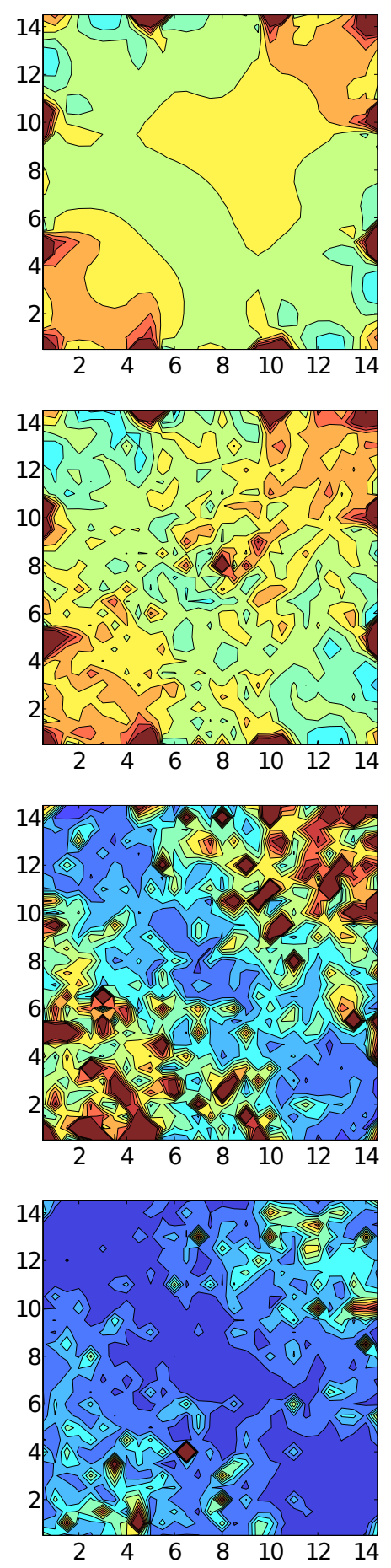

Figure 3.4: Same as Figure 3.3 but using the sinusoid input field shown in Figure 3.1 (right). 


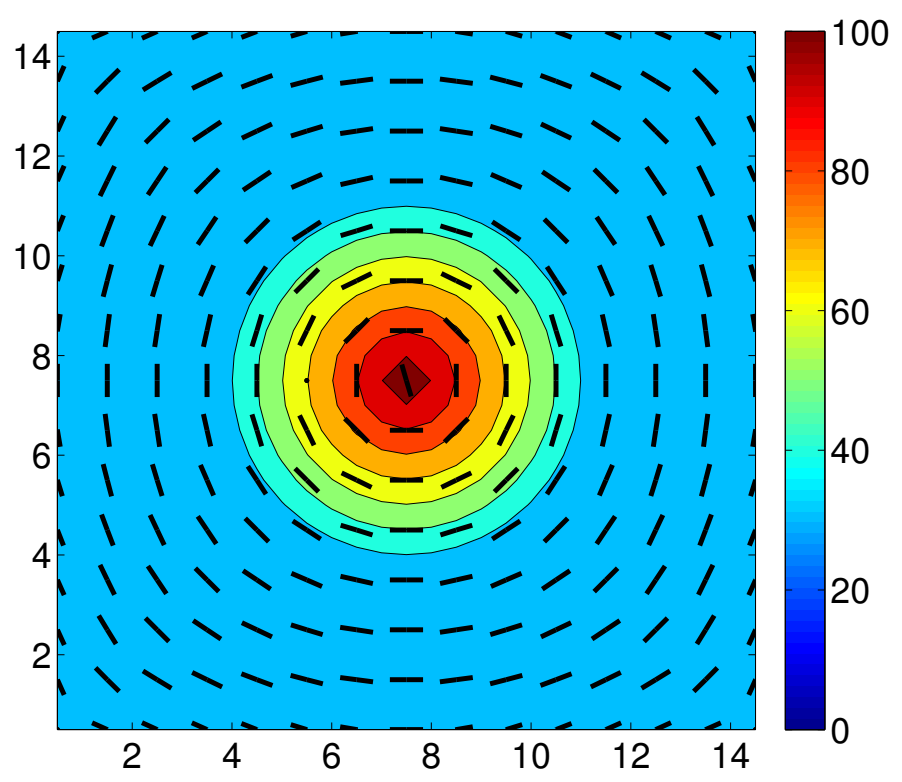

Figure 3.5: Test of the stress method's ability to determine the gravitational stress surrounding a cone of uniform density. The coloured contours are GPE in MPa, which here is a proxy for topographic elevation. The axis units are $100 \mathrm{~km}$. The black bars indicate the direction of the most compressive stress, $S_{H \max }$.

stress is the only non-zero stress and, therefore, corresponds to one of the principal stresses. The other principal axis of stress is perpendicular to this and, thus, circumferential. As the radial stress is extensional, the most compressional stress, $S_{H \max }$, is circumferential. The stress method was able to correctly return the gravitational stress with $S_{H \max }$ circumferential (Fig. 3.5).

Because the Lamb [2015] strain rate method has already been used in New Zealand, I apply the Flesch et al. [2001] stress method to New Zealand using the Quaternary velocity field and GPS-derived velocity field from Section 2.1. The codes used are described in Appendix A. 


\section{Chapter 4}

\section{Results}

The stress method is applied to the New Zealand region to determine the vertically averaged deviatoric stress field. Strain rates calculated from the Quaternary velocity field and the GPS-derived velocity field are used, resulting in two models. A GPE field based on topography and lithospheric density [Lamb, 2015] is used. The GPE field is relative to an arbitrary zero datum. This does not affect the calculation of the stress field as equation (2.7) involves only the gradient of GPE, meaning it is not the absolute magnitude of GPE that is important in the calculation of the deviatoric stress field but rather the variation in GPE. The calculations are performed using finite difference methods between $165.5^{\circ} \mathrm{E}$ and $180^{\circ} \mathrm{E}$ and between $36^{\circ} \mathrm{S}$ and $47^{\circ} \mathrm{S}$. Grid spacing of $0.5^{\circ}$ longitudinally and $0.5^{\circ}$ latitudinally is used throughout the region. This is equivalent to $\sim 40 \mathrm{~km}$ longitudinal spacing and $\sim 50 \mathrm{~km}$ latitudinal spacing.

The results are summarised in Tables 4.1 and 4.2 for the Quaternary velocity field and GPS-derived velocity field, respectively, arranged by the regions specified in Figure 4.2. Only points with a strain rate greater than $0.2 \times 10^{-9} \mathrm{~s}^{-1}\left(0.06 \times 10^{-7} / \mathrm{yr}\right)$ are considered to produce reliable enough results to be included in these comparisons and later figures (see Subsec. 4.1.4). The locations of regions referred to in the descriptions of the results are shown in Figure 4.1. The full results for each region are given in Appendix B. 


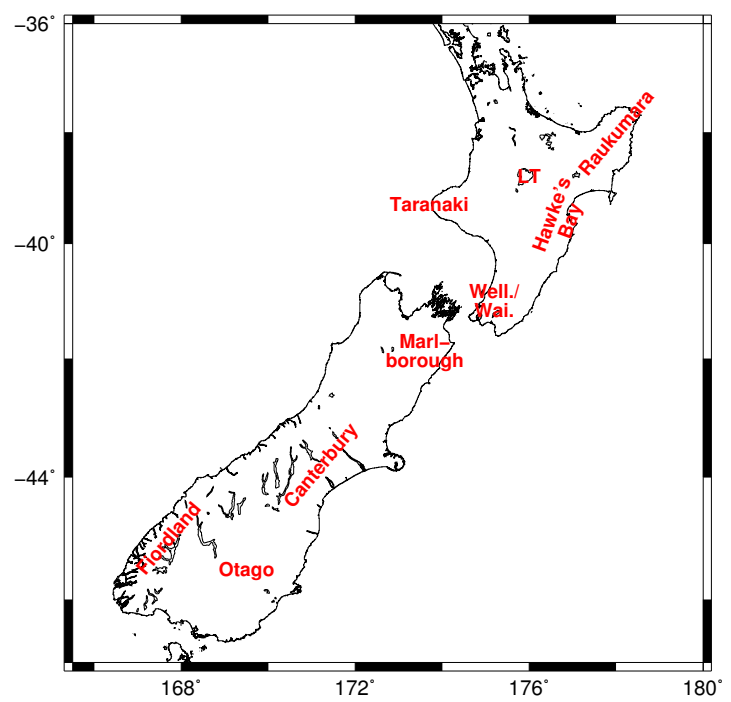

Figure 4.1: Locations of regions that are referred to in the body of the text. LT: Lake Taupo. Well./Wai.: Wellington and Wairarapa regions.

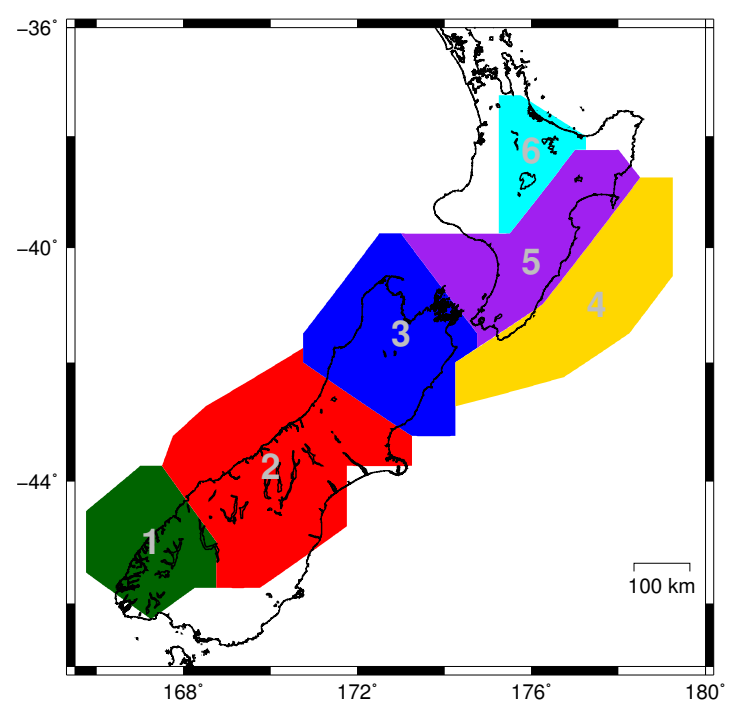

Figure 4.2: Numbered regions used in summary Tables 4.1 and 4.2 .

Table 4.1: Summary of results for different regions (see Fig. 4.2) calculated by applying the Flesch et al. [2001] stress method to Quaternary fault motion data. Dev. Stress $=$ Deviatoric stress; Std. Dev. = Standard deviation. Strain rate and deviatoric stress refer to magnitudes.

\begin{tabular}{ccccccc}
\hline Region & $\begin{array}{c}\text { No. of } \\
\text { Points }\end{array}$ & $\begin{array}{c}\text { Mean } \\
\text { Strain Rate } \\
\left(\times 10^{-7} / \mathrm{yr}\right)\end{array}$ & $\begin{array}{c}\text { Mean Dev. } \\
\text { Stress } \\
(\mathrm{MPa})\end{array}$ & $\begin{array}{c}\text { Std. Dev. } \\
\text { Dev. Stress } \\
(\mathrm{MPa})\end{array}$ & $\begin{array}{c}\text { Mean } \\
\text { Misfit }\end{array}$ & $\begin{array}{c}\text { Median } \\
\text { Viscosity } \\
\left(\times 10^{21} \mathrm{~Pa} \mathrm{~s}\right)\end{array}$ \\
\hline 1 & 20 & 1.70 & 19.5 & 9.2 & 0.15 & 4.9 \\
2 & 46 & 1.07 & 13.3 & 3.5 & 0.14 & 5.4 \\
3 & 32 & 0.90 & 11.2 & 2.7 & 0.09 & 8.5 \\
4 & 34 & 2.29 & 27.3 & 7.2 & 0.03 & 3.9 \\
5 & 30 & 0.86 & 11.1 & 3.3 & 0.27 & 4.2 \\
6 & 12 & 0.80 & 10.6 & 2.6 & 0.18 & 5.2 \\
\hline
\end{tabular}


Table 4.2: Summary of results for different regions (see Fig. 4.2) calculated by applying the Flesch et al. [2001] stress method to the GPS-derived velocity model. Abbreviations as for Table 4.1.

\begin{tabular}{ccccccc}
\hline Region & $\begin{array}{c}\text { No. of } \\
\text { Points }\end{array}$ & $\begin{array}{c}\text { Mean } \\
\text { Strain Rate } \\
\left(\times 10^{-7} / \mathrm{yr}\right)\end{array}$ & $\begin{array}{c}\text { Mean Dev. } \\
\text { Stress } \\
(\mathrm{MPa})\end{array}$ & $\begin{array}{c}\text { Std. Dev. } \\
\text { Dev. Stress } \\
(\mathrm{MPa})\end{array}$ & $\begin{array}{c}\text { Mean } \\
\text { Misfit }\end{array}$ & $\begin{array}{c}\text { Median } \\
\text { Viscosity } \\
\left(\times 10^{21} \text { Pa s }\right)\end{array}$ \\
\hline 1 & 20 & 1.50 & 14.3 & 7.6 & 0.10 & 2.7 \\
2 & 49 & 0.98 & 9.3 & 4.3 & 0.17 & 2.8 \\
3 & 35 & 1.03 & 10.0 & 3.4 & 0.07 & 4.1 \\
4 & 34 & 2.16 & 30.3 & 7.7 & 0.03 & 5.1 \\
5 & 30 & 0.98 & 12.2 & 4.8 & 0.16 & 4.5 \\
6 & 12 & 0.78 & 7.8 & 2.3 & 0.13 & 3.0 \\
\hline
\end{tabular}

\subsection{Stress Results}

\subsubsection{Gravitational Stress Results}

As part of the stress method, the deviatoric stress due to variations in GPE is calculated for the New Zealand region (Fig. 4.3). Gravitational stress magnitudes, taken as the square-root of the second invariant of the gravitational stress tensor, are generally 0-20 MPa. The most extensional gravitational stresses generally correspond to areas of high topography (red in Fig. 4.3), such as the northern South Island and the central North Island. The most compressional gravitational stresses generally correspond to areas of low topography (blue in Fig. 4.3), such as the Hikurangi Plateau to the east of the North Island. Other areas, such as the Chatham Rise, have a mixture of extensional and compressional stress, with one principal gravitational stress extensional and the other compressional. As the calculation of gravitational stress uses only GPE but not strain rates, the Quaternary and GPS-derived models use the same gravitational stress model.

\subsubsection{Total Stress Results}

Using the stress method, the vertically averaged deviatoric stress magnitudes in the New Zealand region are found to be 10-30 MPa (Fig. 4.4). Lower stress magnitudes of 10-20 MPa are found for most of the onshore region, rising to 30-40 MPa near the 


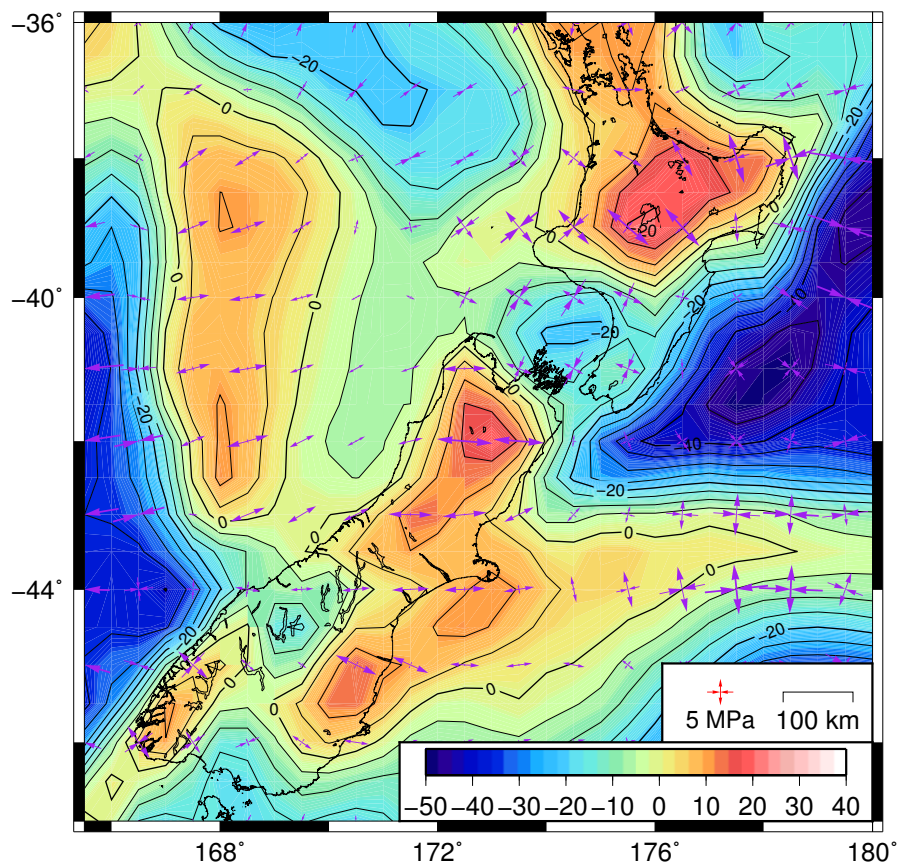

Figure 4.3: Principal axes of deviatoric stress due to gravity calculated as part of the stress method (arrows) from variations in GPE (contours in MPa). Extensional stresses are shown as outwards pointing arrows and compressional stresses are shown as inwards pointing arrows. GPE is from Lamb [2015]; see Subsec. 2.1.3 for details. 

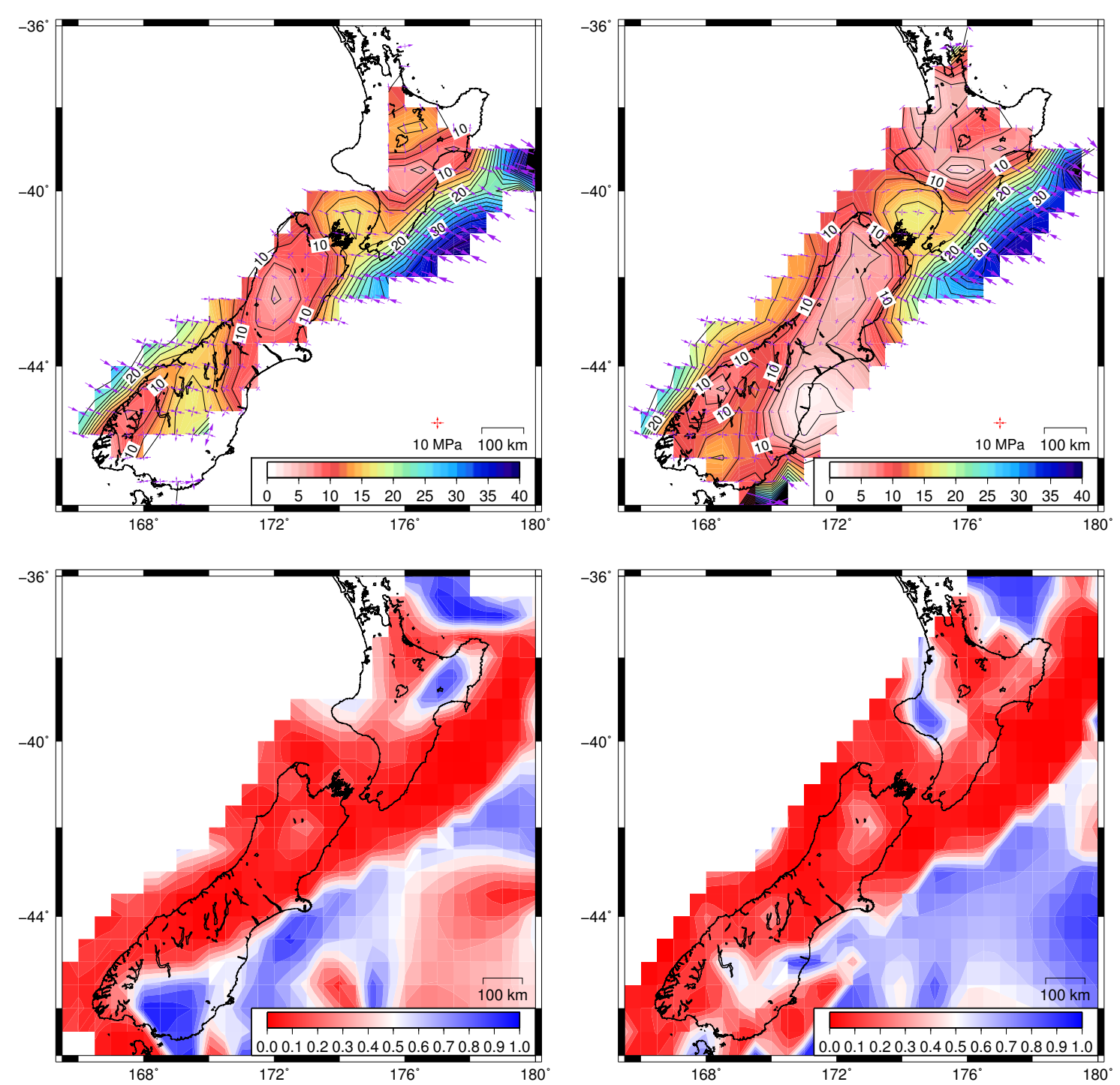

Figure 4.4: Top: Stress magnitudes (square-root of the second invariant; contours) and directions (arrows) in MPa calculated using the Flesch et al. [2001] stress method with strain rates derived from the Quaternary velocity model (left) and GPS-derived velocity model (right). Bottom: Misfit between the orientations of stress and strain rate, calculated using the stress method according to equation (2.13), derived from the Quaternary velocity model (left) and GPS-derived velocity model (right). 
Hikurangi margin. Similarly, in the Quaternary velocity model, the lowest deviatoric stress magnitudes of $\sim 10 \mathrm{MPa}$ are found for the northern South Island and parts of the central North Island. In the GPS-derived model, the low stress magnitudes cover most of the South Island and central North Island. In both models, the lower North Island represents an area of higher stress magnitudes of 15-20 MPa. The deviatoric stress is generally compressional in a northwest-southeast direction for all of New Zealand, reflecting the effect of the boundary stresses that are mostly perpendicular to the plate boundary zone. The sign (extensional or compressional) of the other principal horizontal stress, generally in a northeast-southwest direction, is usually determined by the gravitational stress at that point.

\subsubsection{Boundary Stress Results}

The boundary stresses (Fig. 4.5) in the New Zealand region are compressional with magnitudes of 5-20 MPa. The smallest boundary stresses are determined for the North Island northeast of Lake Taupo and for parts of the southern South Island. The largest boundary stresses are determined for the Wellington and Marlborough regions, near the southern limit of the Hikurangi subduction margin. The Quaternary velocity model also determines greater boundary stresses of 15-20 $\mathrm{MPa}$ for the central South Island, compared to 10-15 MPa determined by the GPS-derived model. Elsewhere, the two models determine comparable magnitudes of boundary stresses, although the boundary stresses for the Quaternary velocity model show greater variation in magnitude than those for the GPS-derived model.

Because the boundary stress is compressional, it partially counteracts the gravitational stress in regions of high topography where the gravitational stress is extensional. Consequently, the regions of high topography and extensional gravitational stress tend to correspond to the regions of lowest overall deviatoric stress magnitudes. This is noticeable in the lower deviatoric stress magnitudes $(<10 \mathrm{MPa})$ determined by both models for the northern South Island and parts of the central North Island; both are regions of significant elevation over a large area. In the northern South Island, the effect of the gravitational stress is so great that in some places, the extensional horizontal stress is larger than the compressional horizontal stress. This indicates that the effect of gravity in determining the stress regime of 

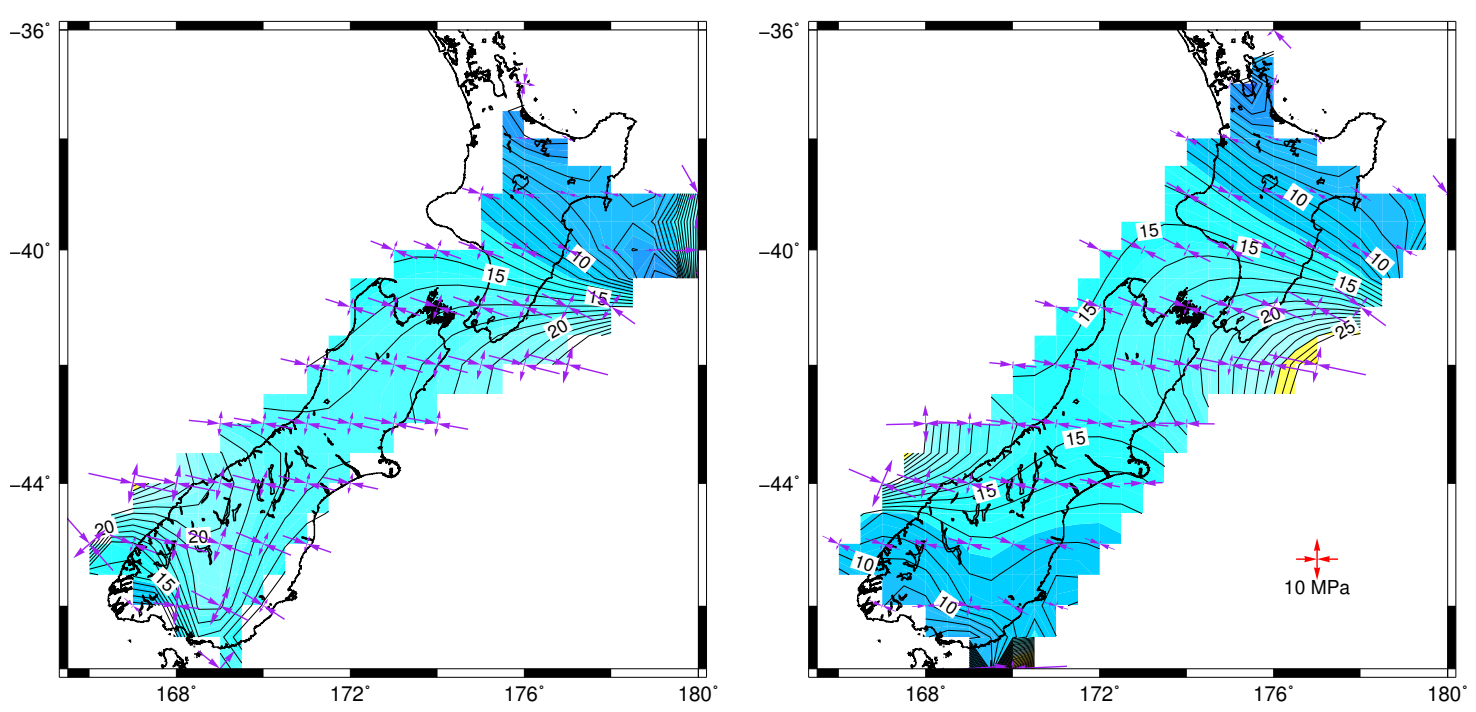

Figure 4.5: The magnitude of the boundary stress component of the deviatoric stress field, calculated using the stress method, derived from the Quaternary velocity model (left) and GPS-derived velocity model (right).

the northern South Island is greater than the effect of plate convergence.

\subsubsection{Misfit}

The areas of lowest misfit (see Fig. 4.4), where the modelled stress directions most closely fit the measured strain rate directions, are the areas of highest strain rate. This is because the areas of higher strain rate contribute more to the minimisation in equation (2.11), with areas of negligible strain rate contributing a negligible amount. Consequently, points with higher strain rates are better constrained in the minimisation than points with low strain rates to the extent that points with very low strain rates may produce unreliable results. For this reason, points with strain rates $<0.2 \times 10^{-9} \mathrm{~s}^{-1}\left(0.06 \times 10^{-7} / \mathrm{yr}\right)$ are not considered reliable enough to be included in this analysis. This includes the assumed rigid parts of the Australian and Pacific plates.

The mean misfit for all regions summarised in Tables 4.1 and 4.2 is $<0.25$, indicating that both models are generally able to match reasonably well the modelled stress orientations with the measured strain rate orientations in these regions. Anomalously high misfits were calculated for the Raukumara Peninsula in the Quaternary 

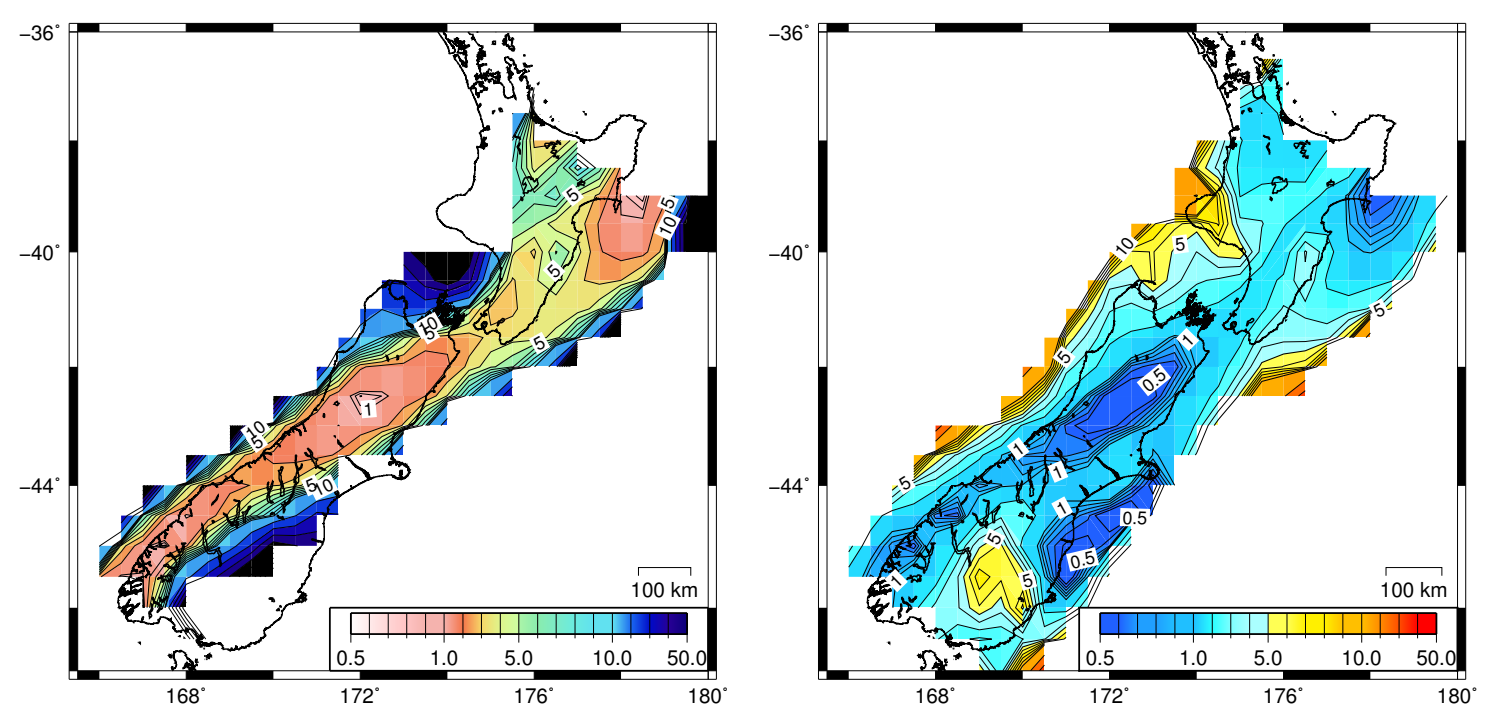

Figure 4.6: Effective viscosity in $\times 10^{21}$ Pas, calculated using the stress method, as the stress magnitude divided by the strain rate magnitude, derived from the Quaternary velocity model (left) and GPS-derived velocity model (right).

velocity model and for Taranaki in the GPS-derived model. A region of anomalously low stress and viscosity, present in the GPS-derived model around coastal Otago and South Canterbury, is centred on a region of high misfit. This suggests that the GPS-derived velocity model is unlikely to be reliable in this region.

\subsection{Viscosity Results}

Effective viscosities (Fig. 4.6), taken as the magnitude of the deviatoric stress divided by the magnitude of the strain rate, are determined for both models. Both models find low viscosities of $1-5 \times 10^{21} \mathrm{~Pa}$ s for the zone of deformation, where the strain rates are greatest. The lowest viscosities of $1-2 \times 10^{21} \mathrm{~Pa}$ s are determined in the South Island, near the Alpine Fault and Marlborough Fault Zone. Viscosities of $2-5 \times 10^{21} \mathrm{~Pa}$ s are determined for the southern North Island. These viscosities increase by an order of magnitude towards the rigid parts of the Australian and Pacific plates. No effective viscosities are calculated for the rigid plates, where the strain rates are modelled as negligible. This is because the calculation of the effective viscosity requires dividing by the magnitude of the strain rate and dividing by a negligibly small strain rate becomes unreliable. Furthermore, the modelled stress in 
these low strain rate regions is considered unreliable (see Subsec. 4.1.4). The GPSderived velocity model gives a wider region of low viscosity than the Quaternary velocity model, consistent with the wider zone of deformation in the GPS-derived velocity model. 


\section{Chapter 5}

\section{Discussion}

\subsection{Comparison to Similar Calculations}

Using the stress method, I determine the vertically averaged deviatoric stress magnitudes in New Zealand to be 10-30 MPa. This is comparable to the vertically averaged deviatoric stress magnitudes of 5-40 MPa found for Tibet [Flesch et al., 2001] using the same method. In western North America, magnitudes of 5-10 MPa were found [Flesch et al., 2007] using a very similar method. The New Zealand magnitudes are also comparable to the $\sim 30 \mathrm{MPa}$ found for northwestern Japan [Yoshida et al., 2015] found by considering the forces needed to match the stress orientations observed from focal mechanisms, following the method of Luttrell et al. [2011].

Gravitational and boundary stresses are found to each account for $\sim 50 \%$ of the deviatoric stresses in New Zealand, indicating that tectonics forces and variations in GPE account for similar proportions of the deviatoric stress in New Zealand. This is comparable to the proportions found in Tibet [Flesch et al., 2001] and western North America [Flesch et al., 2007]. From a force balance perspective, this similarity in magnitudes is unsurprising as it is tectonics that provide the forces to counteract the extensional gravitational stresses in areas of high elevation and it is tectonic forces that create the regions of high elevation. The gravitational stress magnitudes of 0-20 MPa determined for New Zealand are less than the 15-30 MPa determined for Tibet [Ghosh et al., 2009]. This larger magnitude for Tibet is attributable to the greater difference in GPE between Tibet and the Indian Ocean compared to the 
differences in GPE in the New Zealand region.

I determine effective viscosities for the plate boundary zone in New Zealand to be $1-5 \times 10^{21} \mathrm{~Pa}$ s. This is within the range of $10^{20}-10^{22} \mathrm{~Pa}$ s found for plate boundary zones by global dynamical modelling [Ghosh and Holt, 2012]. The effective viscosity here is also comparable to the effective viscosity of $\sim 3 \times 10^{21} \mathrm{~Pa}$ s determined for cross-sections of the Andes using a thin sheet model in two dimensions [Lamb, 2000; Husson and Ricard, 2004]. Overall, the deviatoric stresses and viscosities determined for New Zealand are comparable to the deviatoric stresses and viscosities that have been determined for other plate-boundary zones.

\subsection{Comparison to New Zealand Measurements}

\subsubsection{Comparison between Models}

The Quaternary and GPS-derived velocity models result in generally similar deviatoric stress and effective viscosity fields due to the similarity between the velocity models. The differences between the velocity models are noted in Section 2.1. The similarity between the velocity models indicates that the contemporary GPS-derived velocity field is a good representation of the longer-term Quaternary velocity field, with differences most likely due to elastic strain accumulation as a result of locking on the plate interface [Darby and Beavan, 2001; Lamb and Smith, 2013; Wallace et al., 2009, see Sec. 2.1]. This similarity between seismological and geodetic velocity fields has been observed in New Zealand previously by others [e.g. Walcott, 1984; Nicol and Wallace, 2007]. Litchfield et al. [2014] compared fault slip rates and GPS velocity differences across transects, finding most of the GPS velocity difference to be larger than fault slip rates for 37 out of 47 transects, with the caveat that their fault model may be incomplete in some regions. Larger GPS velocities by $1-5 \mathrm{~mm} / \mathrm{yr}$ in the southern North Island were also found by Litchfield et al. [2014], however, they find correspondingly larger fault slip velocities in the Marlborough Fault Zone by $\sim 6 \mathrm{~mm} / \mathrm{yr}$, a difference that is not present in the models used here.

The similarity between the velocity models is reflected in the similarity between the stress and viscosity fields determined from them. The Quaternary velocity model gives greater deviatoric stress magnitudes for central and northern South Island com- 
pared to the GPS-derived velocities model (regions 2 and 3 in Tables 4.1 and 4.2). This is reflected in the boundary stresses being greater in the Quaternary velocity model for central and northern South Island (the two models use the same gravitational stress model). The GPS-derived velocity model extends the width over which deformation is modelled to occur. This wider zone of deformation is also reflected in the GPS-derived model determining a wider region of low viscosity $\left(<10^{22} \mathrm{~Pa} s\right)$ in the deforming regions, most apparent in the South Island where the deformation is on land and, therefore, well constrained. Despite the difference in the width of the low viscosity zone between the two models, the two models are in strong agreement over the viscosity of the region immediately surrounding the Alpine Fault and Marlborough Fault Zone, as well as between Hawke's Bay and the Hikurangi Margin.

\subsubsection{Comparison between Stress and Strain Rate Methods}

The magnitude range of deviatoric stresses determined in this study is similar, although slightly higher, to that determined by Lamb [2015]. He determined vertically averaged deviatoric stress magnitudes to be 5-20 MPa, compared to 10-30 MPa determined in this study. There are, however, significant differences in the deviatoric stress magnitudes determined for specific parts of New Zealand. In the central North Island, Lamb [2015] determines deviatoric stress of 0-5 MPa, compared to 5-15 MPa determined in this study.

Similarly, while the effective viscosity determined in this study is similar to that determined by Lamb [2015], the viscosities determined by Lamb [2015] tend to be smaller than those determined here. Lamb [2015] determines effective viscosities of $0.1-1 \times 10^{21} \mathrm{~Pa}$ s for the areas of most active deformation, compared to viscosities of $1-5 \times 10^{21} \mathrm{~Pa}$ s determined in this study. This is consistent with the higher deviatoric stresses determined in this study compared to Lamb [2015] as the ratio between the deviatoric stress magnitude and the effective viscosity, i.e. the strain rate, is the same for Lamb [2015] and the Quaternary velocity model of this study.

Overall, this study determines deviatoric stress magnitudes of 10-30 MPa, slightly larger than the 5-20 MPa determined by Lamb [2015]. These differences may be the best estimate of the overall uncertainties in determining the average stress magnitudes from strain rate and GPE data, with uncertainties of the order of 0-10 MPa. 

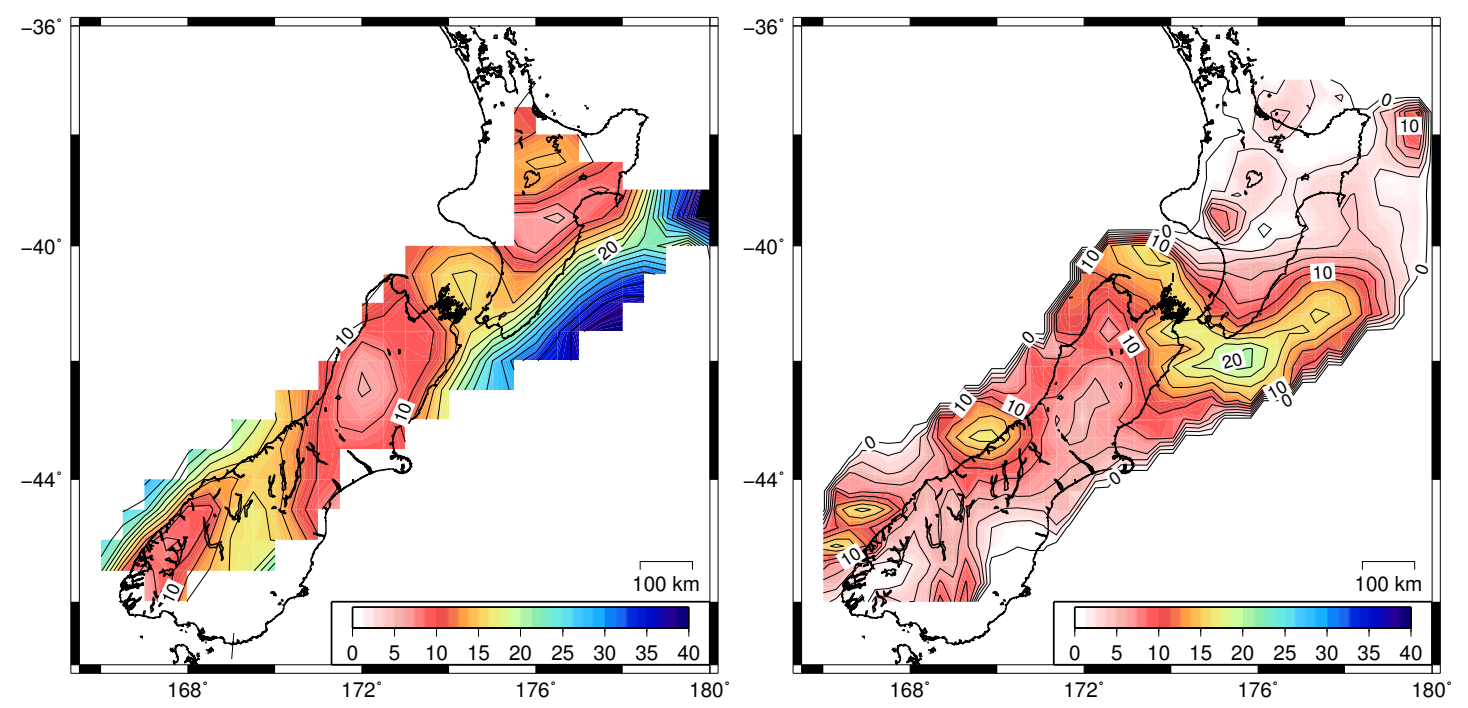

Figure 5.1: Comparison of the stress magnitude calculated in this study using the stress method (left) with those calculated by Lamb [2015] using the strain rate method (right). The two calculations use the same Quaternary velocity model and GPE field.

It should be noted that Flesch et al. [2007] considered their method capable of determining stress magnitudes to within a factor of two, or within $10 \mathrm{MPa}$.

\subsubsection{Comparison to Stress Directions}

One of the assumptions used by both the stress and strain rate methods is of an isotropic effective viscosity, which is equivalent to assuming that the orientations of stress and strain rate are the same. The validity of this assumption can be tested by comparing the orientation of stress in the resultant model with the orientation of stress determined through other methods, such as earthquake focal mechanism inversions. The modelled stress orientations are compared, rather than the orientations of the input strain rates, because the stress method allows deviations from isotropic viscosities, although it seeks to minimise these regions of high misfit.

The direction of most compressive horizontal stress, $S_{\text {Hmax }}$, modelled using the stress method is compared to the direction of $S_{H \max }$ determined by focal mechanism studies [Townend et al., 2012; Balfour et al., 2005; Boese et al., 2012; Warren-Smith, 2016] in Figure 5.2. In the South Island, the modelled directions match well with 


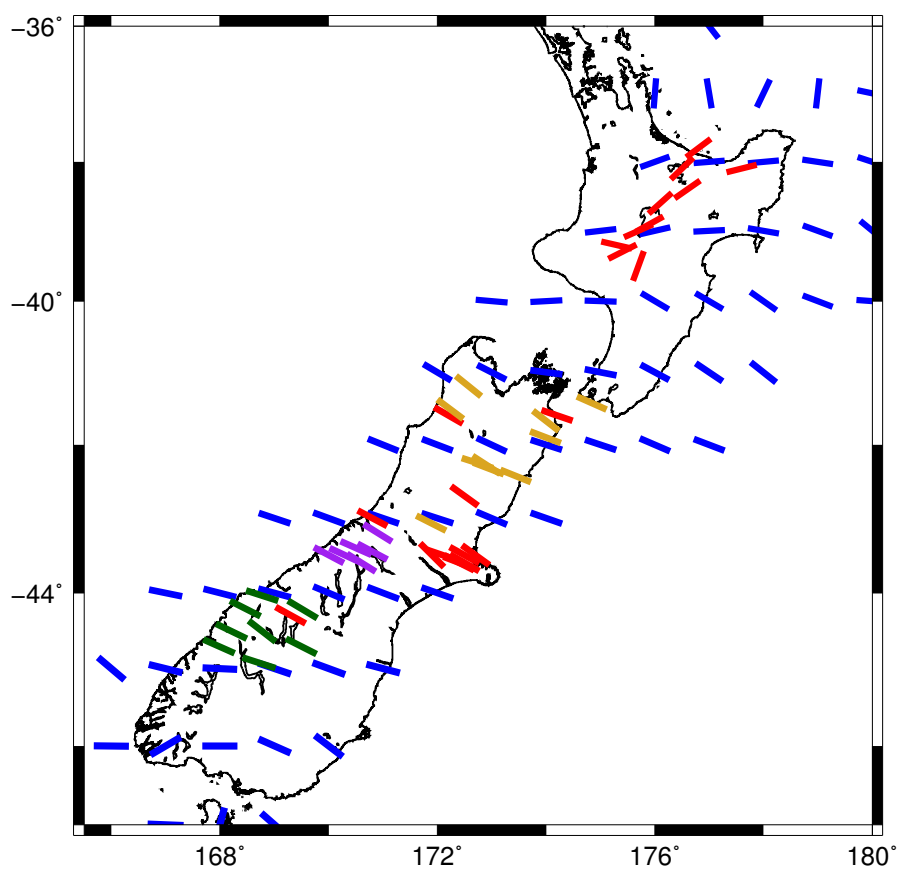

Figure 5.2: Comparison of $S_{H \max }$ directions determined by this study (blue) with directions determined by earthquake focal mechanism studies: Townend et al. [2012, red]; Balfour et al. [2005, gold]; Boese et al. [2012, purple]; Warren-Smith [2016, green].

the measured $S_{H \max }$ directions. In the central South Island, the direction of $S_{H \max }$ is measured as being $110^{\circ}-120^{\circ}$ [Townend et al., 2012; Warren-Smith, 2016; Boese et al., 2012], while the models give an $S_{H \max }$ direction of $\sim 110^{\circ}$. This supports the assumption of an isotropic viscosity being valid. It is harder to compare the modelled orientations of $S_{H \max }$ with those from earthquake focal mechanisms shallower than $20 \mathrm{~km}$ [Townend et al., 2012] in the central North Island due to the greater variation in orientation of $S_{H \max }$ determined from focal mechanisms.

\subsection{Implications of Stress Magnitude}

The vertically averaged deviatoric stress magnitudes of 10-30 MPa determined in this study support the hypothesis that the average stress in the lithosphere is low. Earthquake stress drops are typically in the range of 1-10 MPa [Kanamori and Anderson, 1975], with the $M_{W}=9.02011$ Tohoku earthquake having a stress drop 
of $\sim 20 \mathrm{MPa}$ [Hasegawa et al., 2011], making the deviatoric stress magnitude comparable to the stress drops observed after large earthquakes. This indicates that large earthquakes relieve most of the stress in the crust. This helps to explain why rotations of the stress tensor have been observed after some large earthquakes, such as the 2011 Tohoku earthquake [Hasegawa et al., 2012] and the 1992 Landers earthquake [Hardebeck and Hauksson, 2001].

It is notable that the $M_{W}=7.82016$ Kaikoura earthquake in a region of very low deviatoric stress. The northern South Island has deviatoric stress magnitudes of $<10 \mathrm{MPa}$, with some points as low as $5 \mathrm{MPa}$, amongst the lowest in New Zealand. A typical earthquake stress drop of 1-10 MPa [Kanamori and Anderson, 1975] would remove a large portion of the stress in the region and likely result in rotations of the stress tensor.

The deviatoric stress magnitudes calculated in this study are averaged over the lithosphere to a depth of $100 \mathrm{~km}$. Consequently, it is averaging over the crust and the lithospheric mantle, two rheologically distinct regions. It is, therefore, possible that the crust is stronger, containing a higher stress, and the lithospheric mantle is weaker, containing a lower stress, than the average over the whole lithosphere suggests. Flesch et al. [2007] favoured this interpretation, noting its consistency with extrapolated bore hole stress measurements. Lamb [2015], however, found similar magnitudes of deviatoric stress when using GPE averaged over just the crust (depth of $20 \mathrm{~km}$ ) and over the whole lithosphere (depth of $100 \mathrm{~km}$ ), giving the simple interpretation that the deforming mantle and crust have similar strengths in the order of 10s of MPa. This is because the thickness of the thin sheet only enters the stress balance equation in terms of the right hand term in equation (2.7), which represents the gradient of gravitational potential energy per unit volume (GPE). However, because these gradients of are dominated by surface topography ( $\sim 70 \%$ contribution), they are relatively insensitive to the chosen thickness of the thin sheet over which the stresses are vertically averaged. For this reason, the values of deviatoric stress obtained in this study cannot be explained in terms of averages over a large thickness with much higher stresses in the crust than in the mantle, as suggested by Flesch et al. [2001, 2007], but instead they must represent the general level of stress in the lithosphere [Klein et al., 2009; Lamb, 2015]. 


\subsection{Rheology of the Deforming Zone}

The methodology in this study makes no assumption about the viscous rheology. If the lithology and conditions (i.e., average temperature and pressure) are the same, then ductile flow often exhibits a power-law dependence on strain rate, where the effective viscosity is proportional to $E^{1 / n-1}, E$ is the strain rate magnitude (squareroot of the second invariant of the strain rate tensor), and $n$ is defined as the powerlaw exponent [Ranalli, 1995]. Newtonian fluids have a constant viscosity, regardless of the strain rate, described by a power-law exponent of 1, whereas, silicates such as olivine have a power-law exponent $\sim 3$ [Ranalli, 1995]. For a simple power-law rheology, a log-log plot of strain rate $E$ against effective viscosity $\eta$ should be linear with a slope equal to $1 / n-1$. Figure 5.3 shows such a plot, using the results in this study from the Quaternary velocity field for the whole plate boundary zone, for just the South Island, and for just the North Island.

While the strain rate directions and relative sizes of the strain rate components are used to determine the deviatoric stress, and hence the effective viscosity, the absolute strain rate magnitude is not. This is evidenced by the ability to remove a common factor from all the strain rate components in equation (2.11), changing the magnitude of the strain rate whilst not changing the relative sizes of the components, without altering the stress result obtained from the minimisation. Consequently, the deviatoric stress magnitudes is determined independently of strain rate magnitude, allowing the relationship between strain rate magnitude and either stress magnitude or effective viscosity to be analysed meaningfully.

The best-fitting lines have a slope of $\sim-1$ and imply an inverse relationship between strain rate and viscosity, where the viscosity is approximately proportional to $1 /$ strain rate. The relationship holds when the North and South Islands are considered separately (Fig. 5.3) and is also used empirically in dynamic models of the lithospheric deformation by Wang et al. [2015]. This relationship requires the powerlaw exponent $n$ to be large and, therefore, cannot be explained by a simple powerlaw rheology. However, we would anticipate significant variations in the average temperature of the deforming zone between the relatively 'cold' conditions adjacent to the subduction zones, to the relatively 'hot' conditions in the central North Island, where there is rapid back-arc spreading. Consequently, the viscosity field from the 

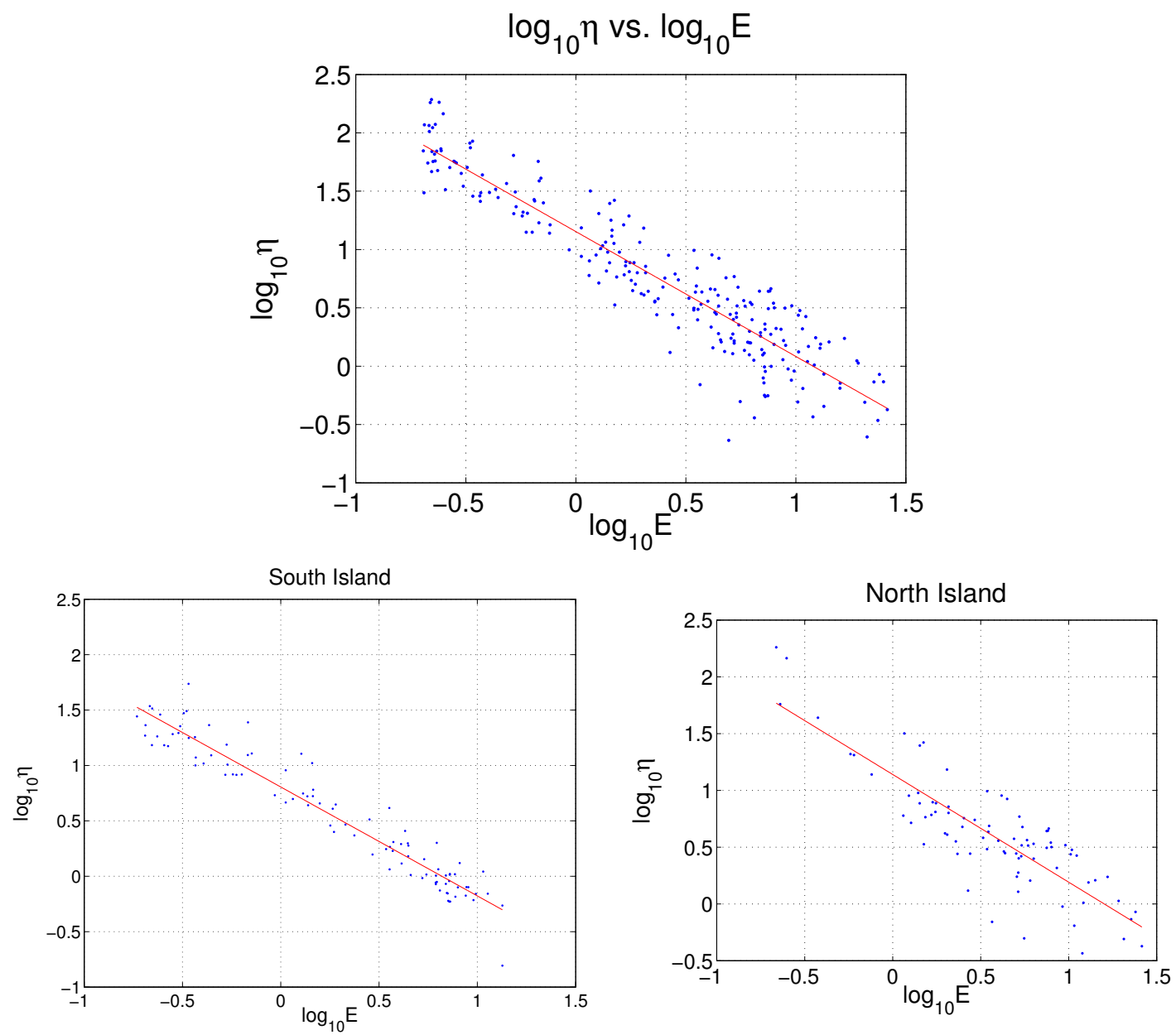

Figure 5.3: Log-log plot of effective viscosity $\eta$ against strain rate magnitude $E$ to test a power-law dependence, using the results from the Quaternary velocity field for the whole plate boundary zone (top), for just the South Island (left), and just the North Island (right). The slopes of the lines are $-1.07,-1.08$, and -0.95 , respectively. 
stress inversion results can be more easily interpreted in terms of the long term strength of the lithosphere, independent of strain rate, but strongly controlled by temperature and/or lithology.

\subsection{Conclusion}

I model the vertically averaged deviatoric stress field in New Zealand from crustal structure and velocity models. I use a thin sheet model of a viscously deforming lithosphere, averaging over a depth of $100 \mathrm{~km}$, and solve the stress balance equation. Two methods of solving the stress balance equation are compared: one method solves first for deviatoric stresses due to gravitational potential energy before accounting for deviatoric stresses due to boundary conditions; the other method assumes an isotropic viscosity to relate deviatoric stress to strain rate, solving for the viscosity field. Under synthetic testing, the two step method is able to cope with high levels of noise but contains edge effects. The method solving for viscosity is accurate at low noise levels, however, it is unreliable at high noise levels.

Using Quaternary fault motion and GPS-derived velocity data, both models predict deviatoric stress magnitudes of 10-30 MPa. This is comparable to what has been found for other plate boundary zones [e.g. Flesch et al., 2001, 2007; Yoshida et al., 2015; Ghosh et al., 2009]. It is also comparable to the stress drops observed after large earthquakes, which helps to explain why rotations of the stress tensor have been observed after some large earthquakes, such as the 2011 Tohoku earthquake [Hasegawa et al., 2012] and the 1992 Landers earthquake [Hardebeck and Hauksson, 2001]. Effective viscosities are found to be $1-20 \times 10^{21} \mathrm{~Pa} \mathrm{~s}$ in the regions of most active deformation, such as the Alpine Fault and Marlborough Fault Zone, and are similarly comparable with effective viscosities found for other plate boundary zones [e.g. Lamb, 2000; Husson and Ricard, 2004; Ghosh and Holt, 2012]. The effective viscosities can be interpreted in terms of the long term strength of the lithosphere controlled by temperature and/or lithology. 


\section{Bibliography}

Altamimi, Z., Métivier, L., and Collilieux, X. (2012). ITRF2008 plate motion model. Journal of Geophysical Research: Solid Earth, 117(B7).

Árnadóttir, T., Thornley, S., Pollitz, F. F., and Darby, D. J. (1999). Spatial and temporal strain rate variations at the northern Hikurangi margin, New Zealand. Journal of Geophysical Research: Solid Earth, 104(B3):4931-4944.

Arnold, R. and Townend, J. (2007). A Bayesian approach to estimating tectonic stress from seismological data. Geophysical Journal International, 170(3):13361356 .

Balfour, N., Savage, M., and Townend, J. (2005). Stress and crustal anisotropy in Marlborough, New Zealand: evidence for low fault strength and structurecontrolled anisotropy. Geophysical Journal International, 163(3):1073-1086.

Beavan, J. and Haines, J. (2001). Contemporary horizontal velocity and strain rate fields of the Pacific-Australian plate boundary zone through New Zealand. Journal of Geophysical Research: Solid Earth, 106(B1):741-770.

Beavan, J., Tregoning, P., Bevis, M., Kato, T., and Meertens, C. (2002). Motion and rigidity of the Pacific Plate and implications for plate boundary deformation. Journal of Geophysical Research: Solid Earth, 107(B10).

Beavan, J., Wallace, L. M., Palmer, N., Denys, P., Ellis, S., Fournier, N., Hreinsdóttir, S., Pearson, C., and Denham, M. (2016). New Zealand GPS velocity field: 1995-2013. New Zealand Journal of Geology and Geophysics, $59(1): 5-14$. 
Boese, C., Townend, J., Smith, E., and Stern, T. (2012). Microseismicity and stress in the vicinity of the Alpine Fault, central Southern Alps, New Zealand. Journal of Geophysical Research: Solid Earth, 117(B2).

Darby, D. and Beavan, J. (2001). Evidence from GPS measurements for contemporary interplate coupling on the southern Hikurangi subduction thrust and for partitioning of strain in the upper plate. Journal of Geophysical Research: Solid Earth, 106(B12):30881-30891.

Engelder, T. (1994). Deviatoric stressitis: A virus infecting the Earth Science community. Eos, Transactions American Geophysical Union, 75(18):209-212.

England, P. and Houseman, G. (1986). Finite strain calculations of continental deformation: 2. Comparison with the India-Asia collision zone. Journal of Geophysical Research: Solid Earth, 91(B3):3664-3676.

England, P. and Jackson, J. (1989). Active deformation of the continents. Annual Review of Earth and Planetary Sciences, 17:197-226.

England, P. and McKenzie, D. (1982). A thin viscous sheet model for continental deformation. Geophysical Journal International, 70(2):295-321.

England, P. and Molnar, P. (1997). Active deformation of Asia: from kinematics to dynamics. Science, 278(5338):647-650.

Flesch, L. M., Haines, A. J., and Holt, W. E. (2001). Dynamics of the India-Eurasia collision zone. Journal of Geophysical Research, 106(B8):16,435-16,460.

Flesch, L. M., Holt, W. E., Haines, A. J., Wen, L., and Shen-Tu, B. (2007). The dynamics of western North America: stress magnitudes and the relative role of gravitational potential energy, plate interaction at the boundary and basal tractions. Geophysics Journal International, 169:866-896.

Ghosh, A. and Holt, W. E. (2012). Plate motions and stresses from global dynamic models. Science, 335(6070):838-843. 
Ghosh, A., Holt, W. E., and Flesch, L. M. (2009). Contribution of gravitational potential energy differences to the global stress field. Geophysical Journal International, 179(2):787-812.

Hardebeck, J. L. and Hauksson, E. (2001). Crustal stress field in southern California and its implications for fault mechanics. Journal of Geophysical Research B, 106(B10):21859-21882.

Hasegawa, A., Yoshida, K., Asano, Y., Okada, T., Iinuma, T., and Ito, Y. (2012). Change in stress field after the 2011 great Tohoku-Oki earthquake. Earth and Planetary Science Letters, 355:231-243.

Hasegawa, A., Yoshida, K., and Okada, T. (2011). Nearly complete stress drop in the 2011 Mw 9.0 off the Pacific coast of Tohoku Earthquake. Earth, planets and space, 63(7):703-707.

Holt, W. E. and Haines, A. (1995). The kinematics of northern South Island, New Zealand, determined from geologic strain rates. Journal of Geophysical Research: Solid Earth, 100(B9):17991-18010.

Houseman, G. and England, P. (1986). Finite strain calculations of continental deformation: 1. Method and general results for convergent zones. Journal of Geophysical Research: Solid Earth, 91(B3):3651-3663.

Husson, L. and Ricard, Y. (2004). Stress balance above subduction: application to the Andes. Earth and Planetary Science Letters, 222(3):1037-1050.

Kanamori, H. and Anderson, D. L. (1975). Theoretical basis of some empirical relations in seismology. Bulletin of the Seismological Society of America, 65(5):1073-1095.

Klein, E. C., Flesch, L. M., Holt, W. E., and Haines, A. J. (2009). Evidence of longterm weakness on seismogenic faults in western North America from dynamic modeling. Journal of Geophysical Research: Solid Earth, 114(B3).

Labuz, J. F. and Zang, A. (2012). Mohr-coulomb failure criterion. Rock Mechanics and Rock Engineering, 45(6):975-979. 
Lamb, S. (2000). Active deformation in the Bolivian Andes, South America. Journal of Geophysical Research: Solid Earth, 105(B11):25627-25653.

Lamb, S. (2015). Kinematics to dynamics in the New Zealand plate boundary zone: implications for the strength of the lithosphere. Geophysical Journal International, 201(2):552-573.

Lamb, S., Mortimer, N., Smith, E., and Turner, G. (2016). Focusing of relative plate motion at a continental transform fault: Cenozoic dextral displacement $>700 \mathrm{~km}$ on new zealand's alpine fault, reversing $>225 \mathrm{~km}$ of late cretaceous sinistral motion. Geochemistry, Geophysics, Geosystems, 17(3):1197-1213.

Lamb, S. and Smith, E. (2013). The nature of the plate interface and driving force of interseismic deformation in the New Zealand plate-boundary zone, revealed by the continuous GPS velocity field. Journal of Geophysical Research: Solid Earth, 118(6):3160-3189.

Lamb, S., Smith, E., Stern, T., and Warren-Smith, E. (2015). Continent-scale strikeslip on a low-angle fault beneath New Zealand's Southern Alps: Implications for crustal thickening in oblique collision zones. Geochemistry, Geophysics, Geosystems, 16(9):3076-3096.

Langridge, R., Ries, W., Litchfield, N., Villamor, P., Van Dissen, R., Barrell, D., Rattenbury, M., Heron, D., Haubrock, S., Townsend, D., et al. (2016). The New Zealand Active Faults Database. New Zealand Journal of Geology and Geophysics, 59(1):86-96.

Litchfield, N., Van Dissen, R., Sutherland, R., Barnes, P., Cox, S., Norris, R., Beavan, R., Langridge, R., Villamor, P., Berryman, K., et al. (2014). A model of active faulting in New Zealand. New Zealand Journal of Geology and Geophysics, 57(1):32-56.

Luttrell, K. M., Tong, X., Sandwell, D. T., Brooks, B. A., and Bevis, M. G. (2011). Estimates of stress drop and crustal tectonic stress from the 27 February 2010 Maule, Chile, earthquake: Implications for fault strength. Journal of Geophysical Research: Solid Earth, 116(B11). 
Mitchell, J., Mackay, K., Neil, H., Mackay, E., Pallentin, A., and Notman, P. (2012). Undersea New Zealand, 1: 5,000,000. NIWA Chart, Miscellaneous series, (92).

Nicol, A. and Wallace, L. (2007). Temporal stability of deformation rates: Comparison of geological and geodetic observations, Hikurangi subduction margin, New Zealand. Earth and Planetary Science Letters, 258(3):397-413.

Rajabi, M., Ziegler, M., Tingay, M., Heidbach, O., and Reynolds, S. (2016). Contemporary tectonic stress pattern of the Taranaki Basin, New Zealand. Journal of Geophysical Research: Solid Earth, 121(8):6053-6070.

Ranalli, G. (1995). Rheology of the Earth. Springer Science \& Business Media.

Savage, M., Aoki, Y., Unglert, K., Ohkura, T., Umakoshi, K., Shimizu, H., Iguchi, M., Tameguri, T., Ohminato, T., and Mori, J. (2016). Stress, strain rate and anisotropy in Kyushu, Japan. Earth and Planetary Science Letters, 439:129 142.

Smith, W. and Wessel, P. (1990). Gridding with continuous curvature splines in tension. Geophysics, 55(3):293-305.

Stein, S. and Wysession, M. (2009). An introduction to seismology, earthquakes, and earth structure. John Wiley \& Sons.

Townend, J., Sherburn, S., Arnold, R., Boese, C., and Woods, L. (2012). Threedimensional variations in present-day tectonic stress along the Australia-Pacific plate boundary in New Zealand. Earth and Planetary Science Letters, 353:4759.

Walcott, R. I. (1978). Present tectonics and late Cenozoic evolution of New Zealand. Geophysical Journal International, 52(1):137-164.

Walcott, R. I. (1984). The kinematics of the plate boundary zone through New Zealand: a comparison of short-and long-term deformations. Geophysical Journal International, 79(2):613-633.

Walcott, R. I. (1998). Modes of oblique compression: Late Cenozoic tectonics of the South Island of New Zealand. Reviews of Geophysics, 36(1):1-26. 
Wallace, L. M., Barnes, P., Beavan, J., Van Dissen, R., Litchfield, N., Mountjoy, J., Langridge, R., Lamarche, G., and Pondard, N. (2012). The kinematics of a transition from subduction to strike-slip: An example from the central New Zealand plate boundary. Journal of Geophysical Research: Solid Earth, $117(\mathrm{~B} 2)$.

Wallace, L. M. and Beavan, J. (2010). Diverse slow slip behavior at the Hikurangi subduction margin, New Zealand. Journal of Geophysical Research: Solid Earth, 115(B12).

Wallace, L. M., Beavan, J., McCaffrey, R., Berryman, K., and Denys, P. (2007). Balancing the plate motion budget in the South Island, New Zealand using GPS, geological and seismological data. Geophysical Journal International, 168(1):332-352.

Wallace, L. M., Beavan, J., McCaffrey, R., and Darby, D. (2004). Subduction zone coupling and tectonic block rotations in the North Island, New Zealand. Journal of Geophysical Research: Solid Earth, 109(B12).

Wallace, L. M., Reyners, M., Cochran, U., Bannister, S., Barnes, P. M., Berryman, K., Downes, G., Eberhart-Phillips, D., Fagereng, A., Ellis, S., et al. (2009). Characterizing the seismogenic zone of a major plate boundary subduction thrust: Hikurangi Margin, New Zealand. Geochemistry, Geophysics, Geosystems, 10(10).

Wang, X., Holt, W. E., and Ghosh, A. (2015). Joint modeling of lithosphere and mantle dynamics: Evaluation of constraints from global tomography models. Journal of Geophysical Research: Solid Earth, 120(12):8633-8655.

Warren-Smith, E. (2016). Lithospheric deformation in the Southern Lakes, New Zealand. PhD thesis, Victoria University of Wellington.

Williams, C. A., Eberhart-Phillips, D., Bannister, S., Barker, D. H., Henrys, S., Reyners, M., and Sutherland, R. (2013). Revised interface geometry for the Hikurangi subduction zone, New Zealand. Seismological Research Letters, 84(6):1066-1073. 
Yoshida, K., Hasegawa, A., and Okada, T. (2015). Spatial variation of stress orientations in NE Japan revealed by dense seismic observations. Tectonophysics, 647:63-72. 


\section{Appendix A}

\section{Codes Used}

Presented below are the codes used to realise the stress method described in Subsection 2.2.2. All programs are written to be run in Matlab. Many are designed to be run in sequence and some cannot be run independently without modification. These files can be accessed at https://github.com/hamishhirschberg/stress-modelling. The versions accessible on Github are the versions intended for real data and differ only in their input and output (but not calculation) from those used for synthetic testing. All of the programs operate with grid points at constant latitude and longitude spacings and use finite differences when calculating derivatives.

\section{A.1 Gravitational Stress}

The gravitational stress was calculated as part of the Flesch et al. [2001] stress method using the program grav_mat_in.m. This program takes as its input a list of gridded points, with their associated longitude, latitude, and GPE. The program calculates the gravitational stress using equation (2.10) at either the input spacing or an increased spacing. The program outputs the calculated gravitational stress as the principal stresses and the azimuth of the most compressive stress, for plotting using GMT's psvelo function. 


\section{A.2 Basis Functions}

Basis functions, representing solutions to the homogeneous version of equation (2.10) (where $\Gamma=0$ ), were calculated as part of the stress method using the program basis_fn_mat many.m. This program uses the region and grid spacing defined during the calculation of gravitational stress. It solves equation (2.10) with $\left(\lambda_{x}, \lambda_{y}\right)$ specified for a given section of the boundary of the region. The program creates a series of basis functions corresponding to the specified sections of the boundary. The basis functions are not outputted outside of Matlab but instead kept as variables within Matlab to be used by later programs (i.e. lin_comb.m) to calculate the full deviatoric stress.

\section{A.3 Full Deviatoric Stress}

The full deviatoric stress calculated using the stress method is calculated by the program lin_comb.m. This program takes as its inputs the gravitational stress and basis functions calculated earlier in the form of Matlab variables. It also takes as input the velocity field, for the same region and grid spacing, that it differentiates to find the strain rate field. The program finds the linear combination of basis functions that when added to the gravitational stress minimise equation (2.11) using the function totlincomb.m. The program outputs a variety of parameters related to the final deviatoric stress solution. These include the principal stresses, azimuth, and magnitude of the gravitational, boundary, and full deviatoric stress and of the input strain rate, as well as the effective viscosity and misfit. The stresses and strain rates outputs are in the format required for plotting using GMT.

\section{A.3.1 Minimisation of Equation (2.11)}

The minimisation of equation (2.11), as part of the stress method, is done by minimising the function totlincomb.m. The function takes as arguments the measured strain rate, the calculated gravitational stress, basis functions, and the coefficient by which each basis function is multiplied. The function returns the value of equation (2.11) for the given combination of strain rate, gravitational stress, basis 
functions, and coefficients. The program lin_comb.m minimises equation (2.11) by varying the coefficients given to totlincomb.m.

\section{A.4 Full Stress Method Implementation}

The full implementation of the stress method is performed by stress_model.m. This program is not responsible for any calculations itself, with the exception of the calculation of the regional summaries. Instead, it calls the above programs in the correct order to allow for simpler implementation. The program also allows for the small differences in the input of the data between the two velocity models. 


\section{Appendix B}

\section{Complete Results}

Presented below in tabular form are the stress and viscosity results from the Quaternary and GPS-derived velocity models, with points arranged by the regions defined in Figure 4.2. The columns headings are abbreviated as follows:

Long. : Longitude of the point;

Lat. : Latitude of the point; $v_{x}:$ East velocity $(\mathrm{mm} / \mathrm{yr})$;

$v_{y}:$ North velocity $(\mathrm{mm} / \mathrm{yr})$;

$\Gamma$ : Gravitational potential energy per unit volume (GPE; in MPa);

$\tau_{e x}:$ Most extensional vertically averaged deviatoric stress (MPa);

$\tau_{c p}:$ Most compressional vertically averaged deviatoric stress (MPa);

$\tau_{a z}:$ Azimuth of the most compressional deviatoric stress;

$\tau_{\text {Gex }}:$ Most extensional gravitational stress (MPa);

$\tau_{G c p}:$ Most compressional gravitational stress (MPa);

$\tau_{\text {Gaz }}$ : Azimuth of the most compressional gravitational stress;

$\eta$ : Effective viscosity $\left(\times 10^{21} \mathrm{~Pa} \mathrm{~s}\right)$;

Mis. : Misfit between stress and strain rate orientations (see Eq. (2.13)). 


\section{B.1 Results of Quaternary Velocity Model}

Below are the stress and viscosity results, with input data, from the Quaternary velocity model in tabular form arranged by region (see Fig. 4.2).

Table B.1: Full results for Region 1 (Fiordland) calculated by applying the Flesch et al. [2001] stress method to the Quaternary velocity model.

\begin{tabular}{ccccccccccccc}
\hline Long. & Lat. & $v_{x}$ & $v_{y}$ & $\Gamma$ & $\tau_{e x}$ & $\tau_{c p}$ & $\tau_{a z}$ & $\tau_{G e x}$ & $\tau_{G c p}$ & $\tau_{G a z}$ & $\eta$ & Mis. \\
\hline 166.0 & -45.0 & -0.1 & 0.0 & -31.5 & 8.3 & -25.9 & 310 & -0.0 & -8.9 & 289 & 25.5 & 0.15 \\
166.0 & -45.5 & -4.2 & -2.0 & -28.4 & -5.4 & -13.3 & 282 & 0.9 & -8.6 & 283 & 2.2 & 0.05 \\
166.5 & -44.5 & -0.0 & 0.0 & -36.9 & 6.1 & -26.1 & 296 & -3.2 & -5.6 & 293 & 49.0 & 0.03 \\
166.5 & -45.0 & -2.0 & -0.3 & -29.5 & 2.9 & -18.9 & 298 & -0.7 & -6.0 & 287 & 4.0 & 0.10 \\
166.5 & -45.5 & -18.6 & -9.2 & -9.7 & 0.6 & -10.6 & 282 & 3.1 & -5.5 & 74 & 1.1 & 0.03 \\
167.0 & -44.0 & -0.0 & 0.0 & -40.2 & 3.0 & -27.6 & 282 & -5.7 & -4.1 & 31 & 109.0 & 0.14 \\
167.0 & -44.5 & -0.9 & 0.4 & -35.9 & 3.3 & -22.3 & 291 & -4.8 & -3.2 & 301 & 8.3 & 0.07 \\
167.0 & -45.0 & -11.6 & -4.4 & -9.9 & 3.5 & -12.5 & 284 & 1.4 & -3.7 & 61 & 1.4 & 0.03 \\
167.0 & -45.5 & -29.8 & -15.6 & 11.3 & 5.2 & -6.6 & 82 & 9.0 & -6.8 & 52 & 1.2 & 0.08 \\
167.0 & -46.0 & -31.6 & -16.5 & 9.8 & 3.2 & -2.7 & 60 & 8.3 & -6.0 & 51 & 0.3 & 0.32 \\
167.5 & -44.0 & -0.2 & 0.2 & -33.3 & 2.5 & -22.6 & 283 & -2.5 & -3.8 & 4 & 21.0 & 0.18 \\
167.5 & -44.5 & -5.9 & -0.9 & -24.4 & 3.3 & -16.5 & 284 & 0.1 & -4.3 & 27 & 2.6 & 0.05 \\
167.5 & -45.0 & -24.5 & -12.8 & 4.0 & 6.7 & -9.2 & 89 & 7.7 & -6.5 & 43 & 1.2 & 0.02 \\
167.5 & -45.5 & -32.5 & -16.9 & 7.4 & 8.9 & -8.8 & 81 & 9.5 & -7.8 & 50 & 5.9 & 0.25 \\
167.5 & -46.0 & -32.6 & -16.6 & -3.1 & 5.6 & -7.8 & 77 & 5.0 & -5.5 & 56 & 9.3 & 0.31 \\
168.0 & -44.5 & -16.6 & -8.2 & -2.9 & 5.8 & -11.1 & 280 & 6.0 & -4.4 & 24 & 1.4 & 0.01 \\
168.0 & -45.0 & -30.4 & -16.4 & 6.2 & 9.4 & -9.7 & 273 & 8.0 & -5.4 & 41 & 3.0 & 0.05 \\
168.0 & -45.5 & -32.7 & -16.4 & -2.7 & 10.8 & -12.8 & 272 & 5.7 & -6.1 & 56 & 24.8 & 0.48 \\
168.5 & -45.0 & -31.8 & -16.2 & -0.2 & 10.4 & -13.0 & 283 & 3.7 & -1.6 & 42 & 8.9 & 0.08 \\
168.5 & -45.5 & -32.6 & -15.9 & -4.0 & 12.4 & -15.4 & 283 & 3.0 & -2.9 & 71 & 46.3 & 0.52 \\
\hline
\end{tabular}


Table B.2: Full results for Region 2 (central South Island) calculated by applying the Flesch et al. [2001] stress method to the Quaternary velocity model.

\begin{tabular}{|c|c|c|c|c|c|c|c|c|c|c|c|c|}
\hline Long. & Lat. & $v_{x}$ & $v_{y}$ & $\Gamma$ & $\tau_{e x}$ & $\tau_{c p}$ & $\tau_{a z}$ & $\tau_{G e x}$ & $\tau_{G c p}$ & $\tau_{G a z}$ & $\eta$ & Mis. \\
\hline 168.0 & -43.5 & -0.0 & 0.0 & -13.2 & 4.3 & -16.2 & 281 & 3.0 & -1.9 & 350 & 61.9 & 0.27 \\
\hline 168.0 & -44.0 & -1.4 & 0.3 & -20.5 & 3.7 & -17.1 & 284 & 1.9 & -3.1 & 3 & 5.1 & 0.16 \\
\hline 168.5 & -43.5 & -0.1 & 0.1 & -9.8 & 5.5 & -14.3 & 285 & 4.5 & -1.3 & 346 & 12.1 & 0.22 \\
\hline 168.5 & -44.0 & -6.3 & -2.3 & -9.4 & 4.8 & -13.0 & 283 & 4.8 & -2.9 & 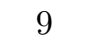 & 2.1 & 0.04 \\
\hline 168.5 & -44.5 & -25.4 & -14.1 & 0.8 & 6.9 & -10.5 & 282 & 6.6 & -3.4 & 22 & 1.8 & 0.01 \\
\hline 169.0 & -43.0 & -0.0 & 0.0 & -0.5 & 7.7 & -11.9 & 289 & 6.8 & -0.3 & 332 & 68.7 & 0.19 \\
\hline 169.0 & -43.5 & -1.0 & -0.2 & -5.5 & 6.8 & -13.0 & 287 & 4.9 & -0.5 & 345 & 3.9 & 0.08 \\
\hline 169.0 & -44.0 & -16.0 & -8.6 & -7.3 & 5.3 & -11.7 & 285 & 5.3 & -2.3 & 9 & 1.6 & 0.02 \\
\hline 169.0 & -44.5 & -29.1 & -15.1 & -14.3 & 6.0 & -13.7 & 286 & 2.8 & -2.4 & 17 & 3.8 & 0.06 \\
\hline 169.0 & -45.0 & -32.4 & -15.8 & -8.2 & 9.6 & -15.5 & 289 & 1.0 & -0.4 & 61 & 18.1 & 0.08 \\
\hline 169.0 & -45.5 & -32.4 & -15.4 & 1.0 & 12.1 & -14.5 & 291 & 3.1 & -1.0 & 77 & 59.3 & 0.54 \\
\hline 169.5 & -43.0 & -0.0 & -0.0 & -0.4 & 8.2 & -12.3 & 290 & 6.3 & -0.2 & 329 & 10.6 & 0.15 \\
\hline 169.5 & -43.5 & -5.7 & -2.9 & -0.2 & 7.7 & -12.0 & 288 & 5.4 & 0.1 & 343 & 2.1 & 0.01 \\
\hline 169.5 & -44.0 & -23.9 & -12.7 & -6.3 & 6.1 & -12.4 & 290 & 4.4 & -1.0 & 357 & 2.1 & 0.02 \\
\hline 169.5 & -44.5 & -31.6 & -15.0 & -15.9 & 5.9 & -14.9 & 291 & 1.3 & -1.2 & 359 & 6.5 & 0.01 \\
\hline 169.5 & -45.0 & -32.7 & -15.2 & -6.0 & 7.9 & -13.3 & 290 & 2.2 & -1.0 & 36 & 30.8 & 0.09 \\
\hline 169.5 & -45.5 & -32.2 & -14.9 & 6.0 & 9.5 & .3 & 29 & 4.8 & -1.0 & 45 & 57.6 & 0.53 \\
\hline 170.0 & -42.5 & 0.0 & 0.0 & -4.4 & 6.7 & -11.8 & 290 & 5.0 & -0.3 & 29 & 65.3 & 0.28 \\
\hline 170.0 & -43.0 & -0.8 & -0.4 & -0.0 & 8.4 & -12.2 & 29 & 5. & -0.0 & 329 & 4.1 & 0.04 \\
\hline 170.0 & -43.5 & -14.7 & -7.9 & 1.0 & 7.8 & -11.2 & 291 & 6.0 & 0.2 & 342 & 1.6 & 0.02 \\
\hline 170.0 & -44.0 & -28.4 & -13.6 & -10.2 & 6.1 & -12.5 & 293 & 4.3 & -0.7 & 349 & 2.8 & 0.01 \\
\hline 170.0 & -44.5 & -33.3 & -14.6 & -6.6 & 6.1 & -11.7 & 293 & 3.8 & -0.7 & 359 & 9.1 & 0.01 \\
\hline 170.0 & -45.0 & -32.9 & -14.5 & 7.3 & 7.2 & -8.0 & 290 & 6.2 & -1.4 & 29 & 38.3 & 0.30 \\
\hline 170.0 & -45.5 & -32.1 & -14.3 & 12.7 & 7.3 & -5 & 29 & 7.5 & -1.5 & 35 & 46.2 & 0.53 \\
\hline 170.5 & -42.5 & -0.0 & -0.0 & -5.1 & 6.5 & -11.8 & 291 & 4. & -0.2 & 332 & 11.2 & 0.16 \\
\hline 170.5 & -43.0 & -5.0 & -2.6 & 0.5 & 8.0 & -11.1 & 29 & 5. & 0. & 3 & 2.0 & 0.02 \\
\hline 170.5 & -43.5 & -22.7 & -11.2 & 6.4 & 8.0 & -9.7 & 29 & 6.7 & 0.6 & 34 & 1.6 & 0.01 \\
\hline 170.5 & -44.0 & -32.3 & -13.8 & -3.2 & 6.1 & -10.2 & 29 & 5.4 & -0.3 & 355 & 3.4 & 0.00 \\
\hline 170.5 & -44.5 & -33.8 & -14.1 & 4.6 & 5.7 & -7.4 & 291 & 6.7 & -0.9 & 11 & 16.6 & 0.06 \\
\hline 170.5 & -45.0 & -33.0 & -13.9 & 14.4 & 6.3 & -4.5 & 289 & 8.6 & -1.4 & 26 & 29.9 & 0.58 \\
\hline 171.0 & -42.5 & -0.9 & -0.3 & -4.1 & 6.5 & -10.7 & 290 & 4. & 0 & 3 & 3.7 & 0.03 \\
\hline 171.0 & -43.0 & -13.9 & -6 & 6.0 & 7. & -8.6 & 289 & 6 & 0.9 & & 1.3 & 0.04 \\
\hline 171.0 & -43.5 & -28.7 & -12.3 & 8.1 & 7.9 & -7.8 & 290 & 7.3 & 0.8 & 3 & 1.8 & 0.05 \\
\hline 171.0 & -44.0 & -34.4 & -13.5 & 1.7 & 6.2 & -8.0 & 291 & 6.6 & -0.0 & 360 & 5.7 & 0.03 \\
\hline 171.0 & -44.5 & -33.9 & -13.4 & 4.9 & 5.2 & -5.8 & 287 & 7.5 & -1.1 & 15 & 30.4 & 0.31 \\
\hline 171.0 & -45.0 & -33.1 & -13.2 & 10.8 & 5.3 & -4.0 & 285 & 8.3 & -1.4 & 25 & 30.5 & 0.60 \\
\hline 171.5 & -42.5 & -5.4 & -2.1 & 2.0 & 6.6 & -7.9 & 288 & 6.3 & 0.4 & 357 & 1.7 & 0.01 \\
\hline 171.5 & -43.0 & -21.5 & -9.0 & 13.3 & 8.0 & -5.9 & 287 & 8.4 & 0. & 1 & 1.3 & 0.08 \\
\hline 171.5 & -43.5 & -33.1 & -12.6 & 9.9 & 8.0 & -7.1 & 290 & 7.7 & 1.0 & & 2.6 & 0.07 \\
\hline 171.5 & -44.0 & -34.8 & -13.0 & 3.3 & 6.5 & -7.3 & 291 & 7.0 & 0.4 & 358 & 16.5 & 0.07 \\
\hline 171.5 & -44.5 & -34.0 & -12.8 & 6.7 & 5.4 & -5.3 & 284 & 7.5 & -0.7 & 16 & 37.2 & 0.51 \\
\hline 172.0 & -43.0 & -26.4 & -9.2 & 13.2 & 7.7 & -5.2 & 288 & 9.1 & 0.6 & 2 & 1.5 & 0.10 \\
\hline 172.0 & -43.5 & -35.1 & -12.3 & 7.1 & 8.0 & -7.7 & 291 & 7.7 & 1.0 & 349 & 4.7 & 0.01 \\
\hline 172.5 & -43.0 & -31.4 & -10.2 & 7.6 & 7.5 & -6.8 & 291 & 8.5 & 0.6 & 355 & 1.9 & 0.05 \\
\hline 172.5 & -43.5 & -35.8 & -11.9 & 6.5 & 8.0 & -8.2 & 291 & 7.6 & 0.9 & 345 & 10.0 & 0.04 \\
\hline 173.0 & -43.5 & -35.9 & -11.3 & 7.8 & 8.4 & -8.6 & 290 & 7.3 & 1.1 & 343 & 35.4 & 0.07 \\
\hline
\end{tabular}


Table B.3: Full results for Region 3 (northern South Island) calculated by applying the Flesch et al. [2001] stress method to the Quaternary velocity model.

\begin{tabular}{|c|c|c|c|c|c|c|c|c|c|c|c|c|}
\hline Long. & Lat. & $v_{x}$ & $v_{y}$ & $\Gamma$ & $\tau_{e x}$ & $\tau_{c p}$ & $\tau_{a z}$ & $\tau_{G}$ & $\tau_{G c p}$ & $\tau_{\text {Gaz }}$ & $\eta$ & Mis. \\
\hline 171.0 & -42.0 & -0.0 & 0.0 & -5.3 & 5.8 & -11.4 & 292 & 4.0 & -0.2 & 336 & 45.2 & 0.02 \\
\hline 171.5 & -41.5 & -0.1 & 0.1 & -5.0 & 5.9 & -10.8 & 295 & 4.3 & -0.1 & 339 & 36.0 & 0.08 \\
\hline 171.5 & -42.0 & -0.4 & 0.2 & -1.3 & 6.1 & -9.8 & 292 & 4.9 & 0.2 & 346 & 8.8 & 0.04 \\
\hline 172.0 & -40.5 & -0.0 & 0.0 & -5.4 & 3.7 & -8.4 & 298 & 4.4 & -0.8 & 1 & 55.5 & 0.05 \\
\hline 172.0 & -41.0 & -0.2 & 0.1 & -1.5 & 5.8 & -8.8 & 301 & 6.2 & -0.1 & 349 & 24.7 & 0.12 \\
\hline 172.0 & -41.5 & -0.5 & 0.3 & 2.1 & 6.6 & -8.1 & 297 & 6.7 & 0.3 & 351 & 16.6 & 0.09 \\
\hline 172.0 & -42.0 & -1.6 & 0.1 & 6.4 & 6.5 & -6.5 & 291 & 7.5 & 0.2 & 2 & 3.1 & 0.01 \\
\hline 172.0 & -42.5 & -12.0 & -4.2 & 10.3 & 6.8 & -4.7 & 286 & 9.2 & -0.2 & 8 & 1.2 & 0.11 \\
\hline 172.5 & -40.5 & -0.3 & 0.1 & -4.7 & 2.1 & -6.7 & 298 & 5.9 & -2.6 & 12 & 20.8 & 0.03 \\
\hline 172.5 & -41.0 & -0.6 & 0.3 & 7.6 & 5.8 & -6.3 & 302 & 8.2 & -0.5 & 359 & 16.5 & 0.23 \\
\hline 172.5 & -41.5 & -1.0 & 0.5 & 15.3 & 8.1 & -5.1 & 299 & 9.9 & 0.7 & 357 & 10.8 & 0.08 \\
\hline 172.5 & -42.0 & -4.5 & -0.5 & 16.1 & 7.7 & -3.7 & 293 & 10.6 & 0.3 & 4 & 2.1 & 0.19 \\
\hline 172.5 & -42.5 & -17.6 & -5.2 & 13.5 & 7.0 & -3.6 & 290 & 10.8 & -0.2 & 6 & 1.2 & 0.15 \\
\hline 173.0 & -40.0 & -0.2 & 0.0 & -7.4 & 0.5 & -6.3 & 275 & 6.4 & -6.7 & 30 & 36.6 & 0.08 \\
\hline 173.0 & -40.5 & -0.6 & 0.2 & -8.8 & -0.1 & -6.1 & 289 & 6.4 & -5.3 & 21 & 23.6 & 0.01 \\
\hline 173.0 & -41.0 & -0.9 & 0.3 & -0.4 & 3.3 & -6.0 & 297 & 7.5 & -2.5 & 11 & 20.0 & 0.06 \\
\hline 173.0 & -41.5 & -1.6 & 0.4 & 13.7 & 7.6 & -4.7 & 298 & 10.1 & 0.1 & 2 & 5.0 & 0.08 \\
\hline 173.0 & -42.0 & -9.2 & -1.8 & 19.9 & 8.7 & -3.3 & 295 & 11.7 & 0.6 & 2 & 1.8 & 0.27 \\
\hline 173.0 & -42.5 & -23.5 & -6.6 & 11.1 & 7.9 & -5.4 & 294 & 10.2 & 0.5 & 357 & 1.4 & 0.11 \\
\hline 173.0 & -43.0 & -35.0 & -10.8 & 4.1 & 7.9 & -9.1 & 293 & 7.6 & 0.5 & 345 & 3.5 & 0.00 \\
\hline 173.5 & -40.5 & -0.8 & 0.1 & -15.7 & -1.2 & -7.7 & 279 & 5.4 & -7.2 & 26 & 39.2 & 0.04 \\
\hline 173.5 & -41.0 & -1.1 & 0.3 & -11.0 & 0.9 & -8.1 & 285 & 5.4 & -5.2 & 22 & 20.4 & 0.17 \\
\hline 173.5 & -41.5 & -3.3 & -0.1 & 4.7 & 5.2 & -6.3 & 287 & 7.8 & -2.1 & 17 & 2.3 & 0.02 \\
\hline 173.5 & -42.0 & -15.1 & -3.5 & 14.6 & 7.9 & -5.4 & 292 & 9.7 & 0.1 & 4 & 1.4 & 0.13 \\
\hline 173.5 & -42.5 & -29.2 & -8.0 & 2.6 & 8.2 & -9.4 & 294 & 7.8 & 0.4 & 346 & 2.0 & 0.03 \\
\hline 173.5 & -43.0 & -36.5 & -10.7 & -0.0 & 8.4 & -11.9 & 292 & 6.3 & 0.1 & 334 & 8.1 & 0.04 \\
\hline 174.0 & -41.0 & -1.4 & 0.2 & -17.7 & 0.1 & -10.2 & 278 & 4.2 & -7.2 & 29 & 10.5 & 0.28 \\
\hline 174.0 & -41.5 & -7.4 & -1.4 & -6.2 & 3.4 & -9.2 & 277 & 6.0 & -5.4 & 29 & 2.1 & 0.04 \\
\hline 174.0 & -42.0 & -21.1 & -5.4 & -0.9 & 5.9 & -9.5 & 288 & 5.6 & -1.1 & 7 & 1.7 & 0.00 \\
\hline 174.0 & -42.5 & -34.1 & -9.3 & -11.1 & 7.5 & -14.4 & 293 & 4.7 & -0.8 & 333 & 4.0 & 0.00 \\
\hline 174.0 & -43.0 & -36.9 & -10.3 & -7.3 & 8.5 & -14.9 & 289 & 4.7 & -0.9 & 322 & 25.6 & 0.16 \\
\hline 174.5 & -41.5 & -13.2 & -3.6 & -12.2 & 2.1 & -11.3 & 275 & 4.8 & -7.1 & 32 & 2.3 & 0.07 \\
\hline
\end{tabular}


Table B.4: Full results for Region 4 (east coast of the North Island to Hikurangi margin) calculated by applying the Flesch et al. [2001] stress method to the Quaternary velocity model.

\begin{tabular}{|c|c|c|c|c|c|c|c|c|c|c|c|c|}
\hline Long. & Lat. & $v_{x}$ & $v_{y}$ & $\Gamma$ & $\tau_{e x}$ & $\tau_{c p}$ & $\tau_{a z}$ & $\tau_{G e x}$ & $\tau_{G c p}$ & $\tau_{G a z}$ & $\eta$ & Mis. \\
\hline 174.5 & -42.0 & -27.1 & -8.5 & -16.1 & 3.6 & -14.3 & 287 & 1.2 & -2.1 & 7 & 3.1 & 0.03 \\
\hline 174.5 & -42.5 & -36.7 & -9.8 & -21.2 & 6.5 & -18.4 & 291 & 2.5 & -2.4 & 324 & 8.7 & 0.02 \\
\hline 175.0 & -42.0 & -31.2 & -10.0 & -30.9 & 1.8 & -18.5 & 288 & -1.9 & -3.0 & 354 & 4.6 & 0.03 \\
\hline 175.0 & -42.5 & -37.6 & -9.3 & -27.0 & 5.3 & -20.6 & 290 & 0.6 & -3.2 & 321 & 15.9 & 0.11 \\
\hline 175.5 & -41.5 & -22.8 & -10.5 & -18.2 & 1.3 & -14.7 & 291 & 1.1 & -4.1 & 11 & 3.4 & 0.03 \\
\hline 175.5 & -42.0 & -35.1 & -9.2 & -38.0 & 1.6 & -21.9 & 291 & -2.8 & -3.8 & 337 & 5.7 & 0.03 \\
\hline 175.5 & -42.5 & -37.9 & -8.8 & -28.1 & 4.9 & -21.5 & 287 & -0.5 & -3.2 & 319 & 39.9 & 0.19 \\
\hline 176.0 & -41.5 & -27.5 & -10.0 & -29.7 & 1.6 & -19.5 & 297 & -1.2 & -3.7 & 350 & 3.4 & 0.01 \\
\hline 176.0 & -42.0 & -37.7 & -8.3 & -42.0 & 2.2 & -24.5 & 293 & -2.4 & -4.6 & 330 & 9.7 & 0.04 \\
\hline 176.5 & -41.0 & -21.6 & -10.5 & -28.8 & 0.8 & -17.3 & 302 & -1.6 & -3.7 & 356 & 3.0 & 0.01 \\
\hline 176.5 & -41.5 & -33.6 & -8.5 & -39.2 & 2.1 & -24.4 & 299 & -2.8 & -4.7 & 334 & 4.3 & 0.00 \\
\hline 176.5 & -42.0 & -38.6 & -7.6 & -42.1 & 3.0 & -26.2 & 293 & -2.4 & -4.9 & 327 & 24.6 & 0.08 \\
\hline 177.0 & -40.5 & -16.0 & -11.0 & -33.2 & -1.1 & -14.6 & 307 & -2.8 & -3.5 & 357 & 2.9 & 0.02 \\
\hline 177.0 & -41.0 & -28.3 & -8.9 & -39.3 & 0.9 & -21.8 & 304 & -4.2 & -4.3 & 337 & 3.2 & 0.00 \\
\hline 177.0 & -41.5 & -38.1 & -7.2 & -45.7 & 2.4 & -27.7 & 299 & -4.1 & -5.5 & 327 & 8.4 & 0.00 \\
\hline 177.0 & -42.0 & -38.8 & -7.1 & -41.2 & 3.7 & -27.6 & 291 & -3.1 & -4.7 & 323 & 146.9 & 0.16 \\
\hline 177.5 & -40.0 & -11.8 & -12.6 & -28.3 & -1.6 & -11.4 & 303 & -2.7 & -2.4 & 325 & 2.0 & 0.00 \\
\hline 177.5 & -40.5 & -22.7 & -9.5 & -40.5 & -1.5 & -17.3 & 309 & .9 & -4.0 & 331 & 2.5 & 0.01 \\
\hline 177.5 & -41.0 & -34.9 & -7.3 & -45.8 & 1.2 & -24.6 & 306 & -5.4 & -5.3 & 325 & 4.4 & 0.01 \\
\hline 177.5 & -41.5 & -39.5 & -6.5 & -48.5 & 2.9 & -29.7 & 299 & -5.2 & -5.8 & 323 & 26.8 & 0.03 \\
\hline 178.0 & -39.5 & -10.5 & -16.5 & -12.8 & 2.4 & -9.7 & 294 & 2.2 & -3.3 & 301 & 1.0 & 0.03 \\
\hline 178.0 & -40.0 & -19.7 & -11.9 & -29.7 & -0.8 & -13.7 & 305 & -1.1 & -4.6 & 310 & 1.5 & 0.00 \\
\hline 178.0 & -40.5 & -30.0 & -7.9 & -43.4 & -0.6 & -19.2 & 313 & -4.2 & -5.7 & 315 & 2.6 & 0.01 \\
\hline 178.0 & -41.0 & -39.2 & -6.1 & -47.9 & 2.4 & -26.2 & 309 & -5.4 & -6.1 & 317 & 8.5 & 0.01 \\
\hline 178.0 & -41.5 & -39.6 & -6.0 & -47.4 & 4.0 & -31.1 & 299 & -5.4 & -5.6 & 319 & 190.0 & 0.13 \\
\hline 178.5 & -39.0 & -15.0 & -17.3 & -17.6 & 3.9 & -12.3 & 284 & 3.3 & -7.1 & 285 & 0.7 & 0.03 \\
\hline 178.5 & -39.5 & -23.0 & -13.4 & -28.1 & 2.1 & -15.4 & 293 & 1.9 & -7.4 & 299 & 1.1 & 0.01 \\
\hline 178.5 & -40.0 & -30.7 & -9.6 & -38.6 & -0.7 & -16.7 & 302 & -0.0 & -7.8 & 305 & 1.7 & 0.00 \\
\hline 178.5 & -40.5 & -37.6 & -6.3 & -46.7 & 0.0 & -19.2 & 314 & -3.0 & -7.5 & 307 & 3.6 & 0.02 \\
\hline 178.5 & -41.0 & -40.3 & -5.4 & -48.2 & 4.0 & -25.9 & 310 & -5.0 & -6.6 & 309 & 29.3 & 0.00 \\
\hline 179.0 & -39.0 & -40.9 & -6.1 & -40.1 & 2.4 & -19.6 & 290 & 1.6 & -12.0 & 289 & 1.6 & 0.00 \\
\hline 179.0 & -39.5 & -42.0 & -5.3 & -44.0 & 2.7 & -22.2 & 292 & 1.1 & -11.7 & 295 & 2.7 & 0.01 \\
\hline 179.0 & -40.0 & -42.0 & -4.9 & -44.5 & -0.3 & -18.8 & 291 & 0.1 & -10.3 & 298 & 4.2 & 0.01 \\
\hline 179.0 & -40.5 & -41.3 & -4.8 & -45.3 & -0.6 & -15.0 & 311 & -2.1 & -8.6 & 298 & 10.7 & 0.08 \\
\hline
\end{tabular}


Table B.5: Full results for Region 5 (southern and eastern North Island) calculated by applying the Flesch et al. [2001] stress method to the Quaternary velocity model.

\begin{tabular}{|c|c|c|c|c|c|c|c|c|c|c|c|c|}
\hline Long. & Lat. & $v_{x}$ & $v_{y}$ & $\Gamma$ & $\tau_{e x}$ & $\tau_{c p}$ & $\tau_{a z}$ & $\tau_{G e x}$ & $\tau_{G c p}$ & $\tau_{G a z}$ & $\eta$ & Mis. \\
\hline 173.5 & -40.0 & -0.3 & 0.0 & -11.3 & 0.4 & -7.3 & 88 & 6.5 & -8.4 & 33 & 47.7 & 0.20 \\
\hline 174.0 & -40.0 & -0.4 & 0.0 & -14.2 & 0.2 & -8.2 & 87 & 6.0 & -8.9 & 34 & 50.9 & 0.28 \\
\hline 174.0 & -40.5 & -1.0 & 0.1 & -21.0 & -1.6 & -9.3 & 278 & 4.1 & -7.8 & 28 & 51.7 & 0.12 \\
\hline 174.5 & -40.0 & -0.5 & 0.1 & -16.4 & -0.2 & -8.3 & 89 & 5.1 & -8.4 & 33 & 51.9 & 0.23 \\
\hline 174.5 & -40.5 & -1.1 & 0.1 & -21.5 & -1.5 & -9.7 & 281 & 3.4 & -7.3 & 26 & 39.6 & 0.12 \\
\hline 174.5 & -41.0 & -2.6 & -0.1 & -16.7 & 0.2 & -10.4 & 278 & .3 & -7.5 & 30 & 5.0 & 0.10 \\
\hline 175.0 & -40.0 & -0.6 & -0.0 & -17.9 & -0.9 & -7.2 & 272 & 5.1 & -7.6 & 29 & 18.9 & 0.11 \\
\hline 175.0 & -40.5 & -1.5 & -0.5 & -21.0 & -1.7 & -8.6 & 283 & 4.2 & -7.3 & 25 & 6.5 & 0.15 \\
\hline 175.0 & -41.0 & -6.9 & -3.3 & -11.3 & 0.5 & -9.1 & 281 & 5.3 & -6.9 & 27 & 2.3 & 0.07 \\
\hline 175.0 & -41.5 & -19.3 & -8.5 & -14.7 & 1.3 & -12.2 & 281 & 3.4 & -6.1 & 26 & 3.0 & 0.08 \\
\hline 175.5 & -40.0 & -1.6 & -2.1 & -11.3 & -0.8 & -4.7 & 282 & 6.3 & -6.0 & 24 & 3.6 & 0.26 \\
\hline 175.5 & -40.5 & -5.6 & -4.8 & -15.9 & -2.0 & -6.6 & 288 & 5.7 & -7 & 23 & 2.6 & 0.17 \\
\hline 175.5 & -41.0 & -13.9 & -8.7 & -10.3 & 0.2 & -8.8 & 288 & 5.2 & -6 & 22 & 2.5 & 0.05 \\
\hline 176.0 & -39.5 & -1.2 & -5.1 & 11.3 & 4.3 & -0.7 & 273 & 8.5 & -2.4 & 27 & 4.4 & 0.52 \\
\hline 176.0 & -40.0 & -4.6 & -6.4 & -5.1 & 0.4 & -3.9 & 301 & 6.3 & -4.0 & 1 & 2.3 & 0.20 \\
\hline 176.0 & -40.5 & -10.8 & -9.1 & -14.6 & -1.1 & -7.3 & 303 & 4.9 & -5.7 & 18 & 3.4 & 0.16 \\
\hline 176.0 & -41.0 & -17.3 & -10.7 & -17.7 & 0.2 & -12.0 & 298 & 2.3 & -4.9 & 14 & 3.5 & 0.02 \\
\hline 176.5 & -39.0 & 1.5 & -10.7 & 15.7 & 8.0 & -1.2 & 78 & 9.8 & -3.4 & 48 & 7.5 & 0.73 \\
\hline 176.5 & -39.5 & -2.2 & -9.5 & -0.0 & 3.0 & -3.0 & 276 & 5 & -2.1 & 3 & 2.8 & 0.42 \\
\hline 176.5 & -40.0 & -6.7 & -9.3 & -14.2 & -0.1 & -6.5 & 303 & 3.0 & -2.9 & 13 & 5.6 & 0.07 \\
\hline 176.5 & -40.5 & -12.4 & -10.8 & -21.8 & -0.4 & -10.9 & 307 & 1.3 & -4.0 & 9 & 4.5 & 0.07 \\
\hline 177.0 & -38.5 & 4.5 & -15.8 & 17.6 & 9.9 & -1.0 & 82 & 10.5 & -3.4 & 66 & 9.6 & 0.83 \\
\hline 177.0 & -39.0 & 0.7 & -14.3 & 6.1 & 7.0 & -3.2 & 87 & 7.1 & -2.8 & 64 & 4.2 & 0.78 \\
\hline 177.0 & -39.5 & -3.3 & -12.7 & -11.1 & 2.0 & -6.2 & 282 & 1.4 & -1.4 & 54 & 3.4 & 0.14 \\
\hline 177.0 & -40.0 & -7.8 & -11.4 & -24.5 & -1.3 & -9.5 & 300 & -1.2 & -2.4 & 10 & 4.1 & 0.01 \\
\hline 177.5 & -38.5 & 3.5 & -19.1 & 14.2 & 9.3 & -2.4 & 86 & 9 & -4.2 & 73 & 6.8 & 0.81 \\
\hline 177.5 & -39.0 & -0.5 & -17.5 & 2.1 & 6.3 & -4.6 & 276 & 5. & -2.4 & 86 & 2.9 & 0.59 \\
\hline 177.5 & -39.5 & -5.0 & -15.7 & -11.8 & 2.0 & -7.5 & 289 & 1.1 & -1.5 & 292 & 1.8 & 0.06 \\
\hline 178.0 & -38.5 & 2.2 & -22.1 & 8.2 & 8.0 & -4.6 & 89 & 8.5 & -5.3 & 77 & 1.9 & 0.48 \\
\hline 178.0 & -39.0 & -2.7 & -20.2 & -1.6 & 5.4 & -6.6 & 280 & 4.7 & -3.3 & 278 & 0.9 & 0.24 \\
\hline
\end{tabular}


Table B.6: Full results for Region 6 (Taupo Volcanic Zone) calculated by applying the Flesch et al. [2001] stress method to the Quaternary velocity model.

\begin{tabular}{ccccccccccccc}
\hline Long. & Lat. & $v_{x}$ & $v_{y}$ & $\Gamma$ & $\tau_{e x}$ & $\tau_{c p}$ & $\tau_{a z}$ & $\tau_{G e x}$ & $\tau_{G c p}$ & $\tau_{G a z}$ & $\eta$ & Mis. \\
\hline 175.5 & -37.5 & 0.0 & -0.0 & 6.4 & 5.7 & -0.7 & 39 & 5.7 & 0.9 & 4 & 12.6 & 0.02 \\
175.5 & -38.0 & 0.0 & -0.1 & 7.8 & 5.8 & -0.4 & 72 & 6.4 & -0.2 & 30 & 6.4 & 0.18 \\
175.5 & -38.5 & 0.1 & -0.2 & 17.0 & 8.6 & -0.7 & 79 & 9.4 & -1.9 & 43 & 6.6 & 0.23 \\
175.5 & -39.0 & 0.2 & -0.6 & 20.4 & 8.7 & -0.6 & 83 & 10.7 & -2.7 & 41 & 7.1 & 0.25 \\
175.5 & -39.5 & -0.4 & -0.9 & 8.0 & 4.4 & -1.9 & 89 & 8.7 & -3.8 & 31 & 4.7 & 0.26 \\
176.0 & -37.5 & 0.8 & -1.7 & 10.8 & 5.3 & 1.1 & 4 & 6.1 & 1.7 & 346 & 2.4 & 0.11 \\
176.0 & -38.0 & 1.6 & -3.4 & 15.2 & 6.1 & 1.8 & 70 & 7.4 & 0.5 & 36 & 2.3 & 0.10 \\
176.0 & -38.5 & 2.2 & -4.7 & 19.2 & 9.1 & 0.2 & 73 & 10.7 & -2.6 & 44 & 3.3 & 0.14 \\
176.0 & -39.0 & 1.7 & -5.5 & 21.0 & 8.5 & 0.3 & 77 & 11.1 & -2.8 & 40 & 5.9 & 0.27 \\
176.5 & -38.0 & 4.9 & -10.3 & 18.9 & 7.5 & 2.2 & 81 & 8.2 & 0.2 & 60 & 2.4 & 0.14 \\
176.5 & -38.5 & 4.5 & -11.2 & 20.1 & 9.9 & -0.0 & 76 & 10.9 & -2.9 & 54 & 5.5 & 0.24 \\
177.0 & -38.0 & 7.1 & -16.5 & 14.9 & 8.2 & 0.6 & 86 & 9.3 & -2.5 & 74 & 4.8 & 0.22 \\
\hline
\end{tabular}




\section{B.2 Results of GPS-Derived Velocity Model}

Below are the stress and viscosity results, with input data, from the GPS-derived velocity model in tabular form arranged by region (see Fig. 4.2).

Table B.7: Full results for Region 1 (Fiordland) calculated by applying the Flesch et al. [2001] stress method to the GPS-derived velocity model.

\begin{tabular}{ccccccccccccc}
\hline Long. & Lat. & $v_{x}$ & $v_{y}$ & $\Gamma$ & $\tau_{e x}$ & $\tau_{c p}$ & $\tau_{a z}$ & $\tau_{G e x}$ & $\tau_{G c p}$ & $\tau_{G a z}$ & $\eta$ & Mis. \\
\hline 166.0 & -45.0 & 0.0 & 0.0 & -31.5 & 3.1 & -19.8 & 293 & -0.0 & -8.9 & 289 & 6.8 & 0.02 \\
166.0 & -45.5 & -5.7 & -1.9 & -28.4 & 3.8 & -16.4 & 288 & 0.9 & -8.6 & 283 & 2.8 & 0.02 \\
166.5 & -44.5 & 0.0 & 0.0 & -36.9 & 5.7 & -18.2 & 301 & -3.2 & -5.6 & 293 & 5.6 & 0.03 \\
166.5 & -45.0 & -7.1 & -0.5 & -29.5 & 2.6 & -16.5 & 289 & -0.7 & -6.0 & 287 & 2.4 & 0.01 \\
166.5 & -45.5 & -14.3 & -4.0 & -9.7 & 4.5 & -9.3 & 278 & 3.1 & -5.5 & 74 & 1.6 & 0.01 \\
167.0 & -44.0 & 0.0 & 0.0 & -40.2 & 5.3 & -23.0 & 293 & -5.7 & -4.1 & 31 & 11.5 & 0.00 \\
167.0 & -44.5 & -6.8 & 0.3 & -35.9 & 1.6 & -15.6 & 297 & -4.8 & -3.2 & 301 & 2.6 & 0.01 \\
167.0 & -45.0 & -16.6 & -0.4 & -9.9 & 2.6 & -9.5 & 278 & 1.4 & -3.7 & 61 & 1.5 & 0.00 \\
167.0 & -45.5 & -19.9 & -5.4 & 11.3 & 6.7 & -4.5 & 78 & 9.0 & -6.8 & 52 & 1.8 & 0.20 \\
167.0 & -46.0 & -22.0 & -7.5 & 9.8 & 5.0 & -3.6 & 78 & 8.3 & -6.0 & 51 & 2.0 & 0.26 \\
167.5 & -44.0 & -3.9 & -0.3 & -33.3 & 2.7 & -18.6 & 293 & -2.5 & -3.8 & 4 & 6.2 & 0.01 \\
167.5 & -44.5 & -13.3 & -1.4 & -24.4 & 0.1 & -10.0 & 289 & 0.1 & -4.3 & 27 & 2.7 & 0.03 \\
167.5 & -45.0 & -22.8 & -4.5 & 4.0 & 4.6 & -5.6 & 76 & 7.7 & -6.5 & 43 & 1.1 & 0.12 \\
167.5 & -45.5 & -23.5 & -7.0 & 7.4 & 7.0 & -5.7 & 70 & 9.5 & -7.8 & 50 & 2.7 & 0.21 \\
167.5 & -46.0 & -25.0 & -7.3 & -3.1 & 2.4 & -6.1 & 76 & 5.0 & -5.5 & 56 & 3.4 & 0.02 \\
168.0 & -44.5 & -16.5 & -5.7 & -2.9 & 1.6 & -4.9 & 278 & 6.0 & -4.4 & 24 & 1.3 & 0.09 \\
168.0 & -45.0 & -30.2 & -8.2 & 6.2 & 5.5 & -5.2 & 77 & 8.0 & -5.4 & 41 & 2.6 & 0.14 \\
168.0 & -45.5 & -26.3 & -10.1 & -2.7 & 5.7 & -8.1 & 78 & 5.7 & -6.1 & 56 & 2.9 & 0.07 \\
168.5 & -45.0 & -28.7 & -10.2 & -0.2 & 5.1 & -7.1 & 275 & 3.7 & -1.6 & 42 & 4.0 & 0.59 \\
168.5 & -45.5 & -29.1 & -10.5 & -4.0 & 6.3 & -9.3 & 272 & 3.0 & -2.9 & 71 & 7.8 & 0.19 \\
\hline
\end{tabular}


Table B.8: Full results for Region 2 (central South Island) calculated by applying the Flesch et al. [2001] stress method to the GPS-derived velocity model.

\begin{tabular}{|c|c|c|c|c|c|c|c|c|c|c|c|c|}
\hline Long. & Lat. & $v_{x}$ & $v_{y}$ & $\Gamma$ & $\tau_{e x}$ & $\tau_{c p}$ & $\tau_{a z}$ & $\tau_{G e x}$ & $\tau_{G c p}$ & $\tau_{G a z}$ & $\eta$ & Mis. \\
\hline 168.0 & -43.5 & -1.1 & 0.1 & -13.2 & 5.3 & -16.6 & 285 & 3.0 & -1.9 & 350 & 12.0 & 0.08 \\
\hline 168.0 & -44.0 & -6.2 & -1.3 & -20.5 & 2.5 & -13.3 & 294 & 1.9 & -3.1 & 3 & 4.6 & 0.16 \\
\hline 168.5 & -43.0 & 0.0 & 0.0 & 1.5 & 6.8 & -11.3 & 281 & 7.1 & -0.1 & 338 & 29.2 & 0.36 \\
\hline 168.5 & -43.5 & -2.1 & 0.6 & -9.8 & 5.0 & -13.5 & 288 & 4.5 & -1.3 & 346 & 7.5 & 0.04 \\
\hline 168.5 & -44.0 & -8.3 & -0.9 & -9.4 & 2.4 & -9.0 & 290 & 4.8 & -2.9 & 9 & 2.2 & 0.08 \\
\hline 168.5 & -44.5 & -20.6 & -7.6 & 0.8 & 2.5 & -4.7 & 279 & 6.6 & -3.4 & 22 & 1.1 & 0.06 \\
\hline 169.0 & -43.0 & 0.0 & 0.0 & -0.5 & 6.6 & -11.4 & 287 & 6.8 & -0.3 & 332 & 13.4 & 0.25 \\
\hline 169.0 & -43.5 & -3.9 & 0.2 & -5.5 & 5.3 & -11.4 & 289 & 4.9 & -0.5 & 345 & 4.4 & 0.07 \\
\hline 169.0 & -44.0 & -14.2 & -3.4 & -7.3 & 2.4 & -7.9 & 289 & 5.3 & -2.3 & 9 & 1.5 & 0.04 \\
\hline 169.0 & -44.5 & -24.6 & -9.2 & -14.3 & 1.6 & -8.5 & 285 & 2.8 & -2.4 & 17 & 3.0 & 0.11 \\
\hline 169.0 & -45.0 & -28.1 & -10.8 & -8.2 & 4.6 & -9.8 & 283 & 1.0 & -0.4 & 61 & 9.6 & 0.52 \\
\hline 169.0 & -45.5 & -28.6 & -12.3 & 1.0 & 7.1 & -8.7 & 280 & 3.1 & -1.0 & 77 & 15.5 & 0.59 \\
\hline 169.5 & -42.5 & 0.0 & 0.0 & -2.3 & 4.7 & -9.8 & 287 & 5.9 & -0.2 & 330 & 31.7 & 0.68 \\
\hline 169.5 & -43.0 & 0.0 & 0.9 & -0.4 & 6.4 & -11.1 & 289 & 6.3 & -0.2 & 329 & 8.4 & 0.08 \\
\hline 169.5 & -43.5 & -5.6 & -0.1 & -0.2 & 5.5 & -10.0 & 290 & 5.4 & 0.1 & 343 & 2.0 & 0.04 \\
\hline 169.5 & -44.0 & -21.7 & -9.8 & -6.3 & 3.0 & -8.9 & 294 & 4.4 & -1.0 & 357 & 1.8 & 0.12 \\
\hline 169.5 & -44.5 & -27.3 & -13.0 & -15.9 & 1.8 & -10.3 & 292 & 1.3 & -1.2 & 359 & 5.5 & 0.10 \\
\hline 169.5 & -45.0 & -28.8 & -12.8 & -6.0 & 3.4 & -8.3 & 285 & 2.2 & -1.0 & 36 & 12.6 & 0.20 \\
\hline 169.5 & -45.5 & -29.1 & -12.9 & 6.0 & 5.5 & -5.5 & 284 & 4.9 & -1.0 & 45 & 12.5 & 0.40 \\
\hline 170.0 & -42.5 & 0.6 & 0.6 & -4.4 & 4.3 & -10.3 & 289 & 5.0 & -0.3 & 329 & 20.4 & 0.25 \\
\hline 170.0 & -43.0 & -1.4 & 1.1 & -0.0 & 6.0 & -10.5 & 290 & 5.9 & -0.0 & 329 & 3.7 & 0.01 \\
\hline 170.0 & -43.5 & -12.2 & -0.9 & 1.0 & 5.1 & -8.9 & 293 & 6.0 & 0.2 & 342 & 1.4 & 0.04 \\
\hline 170.0 & -44.0 & -25.3 & -10.9 & -10.2 & 2.8 & -9.2 & 297 & 4.3 & -0.7 & 349 & 2.0 & 0.30 \\
\hline 170.0 & -44.5 & -29.6 & -14.7 & -6.6 & 2.1 & -7.6 & 296 & 3.8 & -0.7 & 359 & 6.1 & 0.07 \\
\hline 170.0 & -45.0 & -29.4 & -13.2 & 7.3 & 3.0 & -3.7 & 284 & 6.2 & -1.4 & 29 & 3.2 & 0.42 \\
\hline 170.0 & -45.5 & -29.4 & -12.4 & 12.7 & 3.3 & -1.7 & 285 & 7.5 & -1.5 & 35 & 11.9 & 0.73 \\
\hline 170.5 & -42.0 & 0.3 & 0.1 & -5.7 & 2.8 & -10.1 & 291 & 3.8 & -0.3 & 332 & 25.7 & 0.13 \\
\hline 170.5 & -42.5 & -0.1 & 0.4 & -5.1 & 3.8 & -10.2 & 291 & 4.5 & -0.2 & 332 & 8.8 & 0.00 \\
\hline 170.5 & -43.0 & -5.5 & -0.5 & 0.5 & 5.2 & -9.2 & 291 & 5.7 & 0.4 & 334 & 2.0 & 0.01 \\
\hline 170.5 & -43.5 & -21.1 & -8.3 & 6.4 & 4.9 & -7.2 & 295 & 6.7 & 0.6 & 345 & 1.3 & 0.07 \\
\hline 170.5 & -44.0 & -27.3 & -11.0 & -3.2 & 2.6 & -7.0 & 297 & 5.4 & -0.3 & 355 & 2.9 & 0.09 \\
\hline 170.5 & -44.5 & -30.2 & -13.8 & 4.6 & 1.6 & -3.5 & 295 & 6.7 & -0.9 & 11 & 2.6 & 0.02 \\
\hline 170.5 & -45.0 & -30.2 & -12.4 & 14.4 & 2.3 & -0.6 & 276 & 8.6 & -1.4 & 26 & 1.5 & 0.73 \\
\hline 171.0 & -42.5 & -1.9 & -0.8 & -4.1 & 3.5 & -9.0 & 291 & 4.7 & 0.2 & 340 & 3.7 & 0.01 \\
\hline 171.0 & -43.0 & -11.1 & -3.4 & 6.0 & 4.6 & -6.5 & 290 & 6.5 & 0.9 & 348 & 1.1 & 0.01 \\
\hline 171.0 & -43.5 & -25.8 & -10.4 & 8.1 & 4.3 & -5.1 & 293 & 7.3 & 0.8 & 354 & 1.4 & 0.00 \\
\hline 171.0 & -44.0 & -30.3 & -11.8 & 1.7 & 2.4 & -4.8 & 296 & 6.6 & -0.0 & 360 & 2.4 & 0.03 \\
\hline 171.0 & -44.5 & -31.6 & -12.0 & 4.9 & 1.0 & -2.1 & 287 & 7.5 & -1.1 & 15 & 1.6 & 0.02 \\
\hline 171.0 & -45.0 & -32.5 & -12.7 & 10.8 & 1.7 & -0.7 & 85 & 8.3 & -1.4 & 25 & 0.9 & 0.70 \\
\hline 171.5 & -42.5 & -5.1 & -0.5 & 2.0 & 3.3 & -6.1 & 289 & 6.3 & 0.4 & 357 & 1.8 & 0.01 \\
\hline 171.5 & -43.0 & -18.6 & -5.3 & 13.3 & 4.3 & -3.6 & 288 & 8.4 & 0.9 & 1 & 0.8 & 0.07 \\
\hline 171.5 & -43.5 & -28.8 & -11.2 & 9.9 & 4.0 & -4.3 & 293 & 7.7 & 1.0 & 354 & 1.5 & 0.01 \\
\hline 171.5 & -44.0 & -31.7 & -11.9 & 3.3 & 2.3 & -4.0 & 298 & 7.0 & 0.4 & 358 & 2.8 & 0.03 \\
\hline 171.5 & -44.5 & -32.4 & -11.5 & 6.7 & 0.9 & -1.6 & 283 & 7.5 & -0.7 & 16 & 1.3 & 0.10 \\
\hline 172.0 & -43.0 & -24.3 & -9.0 & 13.2 & 3.7 & -2.8 & 289 & 9.1 & 0.6 & 2 & 0.8 & 0.06 \\
\hline 172.0 & -43.5 & -31.8 & -11.3 & 7.1 & 3.5 & -4.7 & 296 & 7.7 & 1.0 & 349 & 2.7 & 0.00 \\
\hline 172.5 & -43.0 & -28.5 & -11.4 & 7.6 & 3.1 & -4.2 & 293 & 8.5 & 0.6 & 355 & 1.3 & 0.01 \\
\hline 172.5 & -43.5 & -32.0 & -11.7 & 6.5 & 3.0 & -4.8 & 295 & 7.6 & 0.9 & 345 & 4.1 & 0.08 \\
\hline 173.0 & -43.5 & -33.4 & -11.1 & 7.8 & 2.8 & -4.8 & 288 & 7.3 & 1.1 & 343 & 2.0 & 0.10 \\
\hline
\end{tabular}


Table B.9: Full results for Region 3 (northern South Island) calculated by applying the Flesch et al. [2001] stress method to the GPS-derived velocity model.

\begin{tabular}{|c|c|c|c|c|c|c|c|c|c|c|c|c|}
\hline Long. & Lat. & $v_{x}$ & $v_{y}$ & $\Gamma$ & $\tau_{e x}$ & $\tau_{c p}$ & $\tau_{a z}$ & $\tau_{G e x}$ & $\tau_{G c p}$ & $\tau_{G a z}$ & $\eta$ & Mis. \\
\hline 171.0 & -41.5 & -0.5 & -0.2 & -5.0 & 2.8 & -10.2 & 295 & 3.7 & -0.3 & 332 & 11.5 & 0.07 \\
\hline 171.0 & -42.0 & -0.7 & -0.2 & -5.3 & 2.9 & -9.9 & 293 & 4.0 & -0.2 & 336 & 6.7 & 0.05 \\
\hline 171.5 & -41.0 & -0.8 & -0.2 & -5.4 & 2.5 & -9.6 & 301 & 3.9 & -0.2 & 341 & 11.5 & 0.03 \\
\hline 171.5 & -41.5 & -2.2 & -0.4 & -5.0 & 3.1 & -9.7 & 298 & 4.3 & -0.1 & 339 & 7.3 & 0.03 \\
\hline 171.5 & -42.0 & -3.4 & -0.2 & -1.3 & 3.1 & -8.4 & 294 & 4.9 & 0.2 & 346 & 4.7 & 0.03 \\
\hline 172.0 & -40.5 & -0.8 & 0.0 & -5.4 & 1.4 & -8.1 & 306 & 4.4 & -0.8 & 1 & 8.8 & 0.03 \\
\hline 172.0 & -41.0 & -2.0 & -0.2 & -1.5 & 3.5 & -8.4 & 307 & 6.2 & -0.1 & 349 & 6.2 & 0.06 \\
\hline 172.0 & -41.5 & -3.5 & -0.0 & 2.1 & 3.9 & -7.2 & 301 & 6.7 & 0.3 & 351 & 4.3 & 0.08 \\
\hline 172.0 & -42.0 & -4.6 & -0.0 & 6.4 & 3.4 & -5.1 & 293 & 7.5 & 0.2 & 2 & 2.9 & 0.02 \\
\hline 172.0 & -42.5 & -8.8 & -1.9 & 10.3 & 3.3 & -2.8 & 285 & 9.2 & -0.2 & 8 & 0.7 & 0.07 \\
\hline 172.5 & -40.0 & -0.7 & 0.1 & -5.4 & -1.5 & -6.0 & 297 & 5.4 & -4.3 & 27 & 11.4 & 0.03 \\
\hline 172.5 & -40.5 & -2.2 & 0.3 & -4.7 & 0.1 & -6.9 & 310 & 5.9 & -2.6 & 12 & 8.4 & 0.03 \\
\hline 172.5 & -41.0 & -3.7 & 0.3 & 7.6 & 3.7 & -6.3 & 310 & 8.2 & -0.5 & 359 & 5.4 & 0.07 \\
\hline 172.5 & -41.5 & -5.7 & 0.4 & 15.3 & 5.5 & -4.6 & 305 & 9.9 & 0.7 & 357 & 3.6 & 0.28 \\
\hline 172.5 & -42.0 & -7.4 & -0.2 & 16.1 & 4.5 & -2.5 & 297 & 10.6 & 0.3 & 4 & 1.3 & 0.17 \\
\hline 172.5 & -42.5 & -16.7 & -7.2 & 13.5 & 3.3 & -1.7 & 291 & 10.8 & -0.2 & 6 & 0.5 & 0.22 \\
\hline 173.0 & -40.0 & -1.2 & 0.2 & -7.4 & -2.5 & -5.6 & 285 & 6.4 & -6.7 & 30 & 12.1 & 0.04 \\
\hline 173.0 & -40.5 & -2.5 & 0.3 & -8.8 & -2.3 & -6.1 & 306 & 6 & -5.3 & 21 & 12.3 & 0.07 \\
\hline 173.0 & -41.0 & -4.2 & 0.2 & -0.4 & 1.1 & -6.0 & 306 & 7.5 & -2.5 & 11 & 6.4 & 0.01 \\
\hline 173.0 & -41.5 & -7.0 & 0.3 & 13.7 & 5.0 & -4.2 & 303 & 10.1 & 0.1 & 2 & 2.6 & 0.20 \\
\hline 173.0 & -42.0 & -12.4 & -1.9 & 19.9 & 5.5 & -2.2 & 299 & 11.7 & 0.6 & 2 & 1.1 & 0.37 \\
\hline 173.0 & -42.5 & -23.3 & -8.3 & 11.1 & 4.0 & -3.6 & 296 & 10.2 & 0.5 & 357 & 0.8 & 0.09 \\
\hline 173.0 & -43.0 & -31.1 & -11.4 & 4.1 & 3.2 & -6.3 & 294 & 7.6 & 0.5 & 345 & 2.2 & 0.00 \\
\hline 173.5 & -40.5 & -3.4 & 0.1 & -15.7 & -3.8 & -7.5 & 289 & 5.4 & -7.2 & 26 & 8.7 & 0.06 \\
\hline 173.5 & -41.0 & -5.6 & -0.4 & -11.0 & -1.6 & -7.9 & 289 & 5. & -5.2 & 22 & 5.1 & 0.06 \\
\hline 173.5 & -41.5 & -10.4 & -1.5 & 4.7 & 2.5 & -5.8 & 288 & 7.8 & -2.1 & 17 & 1.8 & 0.01 \\
\hline 173.5 & -42.0 & -20.5 & -6.1 & 14.6 & 4.7 & -4.4 & 292 & 9.7 & 0.1 & 4 & 1.1 & 0.09 \\
\hline 173.5 & -42.5 & -28.7 & -10.3 & 2.6 & 4.1 & -7.6 & 294 & 7.8 & 0.4 & 346 & 1.9 & 0.01 \\
\hline 173.5 & -43.0 & -34.2 & -10.5 & -0.0 & 3.4 & -9.1 & 290 & 6.3 & 0.1 & 334 & 2.7 & 0.03 \\
\hline 174.0 & -41.0 & -8.8 & -1.1 & -17.7 & -2.3 & -10.2 & 279 & 4.2 & -7.2 & 29 & 4.1 & 0.07 \\
\hline 174.0 & -41.5 & -14.8 & -4.0 & -6.2 & 0.9 & -9.2 & 275 & 6.0 & -5.4 & 29 & 2.2 & 0.05 \\
\hline 174.0 & -42.0 & -24.6 & -8.0 & -0.9 & 2.8 & -9.0 & 285 & 5.6 & -1.1 & 7 & 2.0 & 0.02 \\
\hline 174.0 & -42.5 & -32.4 & -9.3 & -11.1 & 3.5 & -12.8 & 290 & 4.7 & -0.8 & 333 & 3.1 & 0.01 \\
\hline 174.0 & -43.0 & -36.8 & -8.5 & -7.3 & 3.6 & -12.3 & 284 & 4.7 & -0.9 & 322 & 4.2 & 0.06 \\
\hline 174.5 & -41.5 & -21.9 & -6.7 & -12.2 & -0.0 & -11.7 & 273 & 4.8 & -7.1 & 32 & 2.2 & 0.02 \\
\hline
\end{tabular}


Table B.10: Full results for Region 4 (east coast of the North Island to Hikurangi margin) calculated by applying the Flesch et al. [2001] stress method to the GPSderived velocity model.

\begin{tabular}{|c|c|c|c|c|c|c|c|c|c|c|c|c|}
\hline Long. & Lat. & $v_{x}$ & $v_{y}$ & $\Gamma$ & $\tau_{e x}$ & $\tau_{c p}$ & $\tau_{a z}$ & $\tau_{\text {Gex }}$ & $\tau_{G c p}$ & $\tau_{G a z}$ & $\eta$ & Mis. \\
\hline 174.5 & -42.0 & -30.3 & -8.1 & -16.1 & 0.9 & -14.2 & 283 & 1.2 & -2.1 & 7 & 3.0 & 0.01 \\
\hline 174.5 & -42.5 & -36.5 & -7.6 & -21.2 & 2.7 & -17.3 & 287 & 2.5 & -2.4 & 324 & 4.5 & 0.01 \\
\hline 175.0 & -42.0 & -35.8 & -8.2 & -30.9 & -0.6 & -18.9 & 285 & -1.9 & -3.0 & 354 & 5.5 & 0.01 \\
\hline 175.0 & -42.5 & -39.9 & -6.3 & -27.0 & 2.0 & -20.1 & 284 & 0.6 & -3.2 & 21 & 10.5 & 0.03 \\
\hline 175.5 & -41.5 & -31.9 & -9.7 & -18.2 & -0.4 & -15.9 & 290 & 1.1 & -4.1 & 11 & 6.7 & 0.06 \\
\hline 175.5 & -42.0 & -37.2 & -7.8 & -38.0 & -0.6 & -22.8 & 288 & -2.8 & -3.8 & 337 & 10.6 & 0.08 \\
\hline 175.5 & -42.5 & -39.8 & -5.8 & -28.1 & 2.3 & -22.1 & 281 & -0.5 & -3.2 & 319 & 29.4 & 0.28 \\
\hline 176.0 & -41.5 & -33.7 & -9.5 & -29.7 & 0.1 & -21.2 & 296 & -1.2 & -3.7 & 350 & 7.3 & 0.03 \\
\hline 176.0 & -42.0 & -38.1 & -7.1 & -42.0 & 0.4 & -26.1 & 290 & -2.4 & -4.6 & 330 & 12.1 & 0.08 \\
\hline 176.5 & -41.0 & -29.3 & -11.3 & -28.8 & -0.2 & -19.3 & 303 & -1.6 & -3.7 & 56 & 5.4 & 0.01 \\
\hline 176.5 & -41.5 & -35.8 & -8.4 & -39.2 & 1.0 & -26.5 & 298 & -2.8 & -4.7 & 334 & 7.2 & 0.02 \\
\hline 176.5 & -42.0 & -39.3 & -5.9 & -42.1 & 1.7 & -28.7 & 289 & -2.4 & -4.9 & 327 & 13.3 & 0.06 \\
\hline 177.0 & -40.5 & -21.6 & -14.5 & -33.2 & -1.8 & -16.9 & 309 & -2.8 & -3.5 & 357 & 3.7 & 0.04 \\
\hline 177.0 & -41.0 & -31.7 & -10.2 & -39.3 & -0.1 & -23.8 & 305 & -4.2 & -4.3 & 337 & 4.6 & 0.01 \\
\hline 177.0 & -41.5 & -38.6 & -6.2 & -45.7 & 1.6 & -30.2 & 298 & -4.1 & -5.5 & 327 & 7.2 & 0.01 \\
\hline 177.0 & -42.0 & -40.8 & -4.2 & -41.2 & 3.1 & -31.2 & 288 & -3.1 & -4.7 & 323 & 26.3 & 0.06 \\
\hline 177.5 & -40.0 & -15.4 & -16.0 & -28.3 & -1.8 & -13.6 & 309 & -2.7 & -2.4 & 325 & 2.1 & 0.01 \\
\hline 177.5 & -40.5 & -26.1 & -12.1 & -40.5 & -2.4 & -19.6 & 31 & -4.9 & -4.0 & 3 & 3.1 & 0.01 \\
\hline 177.5 & -41.0 & -35.6 & -7.5 & -45.8 & -0.1 & -26.4 & 307 & -5.4 & -5.3 & 32 & 4.8 & 0.01 \\
\hline 177.5 & -41.5 & -41.8 & -3.7 & -48.5 & 2.2 & -32.4 & 29 & -5.2 & -5.8 & 323 & 13.3 & 0.02 \\
\hline 178.0 & -39.5 & -13.6 & -15.7 & -12.8 & 1.8 & -11.0 & 302 & 2.2 & -3.3 & 301 & 1.3 & 0.00 \\
\hline 178.0 & -40.0 & -23.0 & -12.5 & -29.7 & -0.7 & -16.1 & 309 & -1.1 & -4.6 & 0 & 2.0 & 0.01 \\
\hline 178.0 & -40.5 & -32.2 & -8.6 & -43.4 & -2.0 & -21.4 & 311 & -4.2 & -5.7 & 3 & 3.2 & 0.01 \\
\hline 178.0 & -41.0 & -39.1 & -5.1 & -47.9 & 0.4 & -27.3 & 30 & -5 & -6.1 & & 6.2 & 0.00 \\
\hline 178.0 & -41.5 & -41.7 & -3.2 & -47.4 & 3.1 & -33.4 & 30 & -5.4 & -5.6 & 3 & 46.5 & 0.13 \\
\hline 178.5 & -39.0 & -14.1 & -15.1 & -17.6 & 2.4 & -12.7 & 292 & 3.3 & -7.1 & 285 & 1.1 & 0.01 \\
\hline 178.5 & -39.5 & -23.8 & -11.6 & -28.1 & 1.6 & -16.6 & 299 & 1.9 & -7.4 & 299 & 1.6 & 0.00 \\
\hline 178.5 & -40.0 & -32.0 & -8.2 & -38.6 & -0.0 & -19.5 & 304 & -0.0 & -7.8 & 305 & 2.3 & 0.00 \\
\hline 178.5 & -40.5 & -38.9 & -4.9 & -46.7 & -1.8 & -21.5 & 309 & -3.0 & -7.5 & 307 & 3.8 & 0.01 \\
\hline 178.5 & -41.0 & -42.7 & -2.7 & -48.2 & 0.6 & -25.3 & 310 & -5.0 & -6.6 & 309 & 12.4 & 0.00 \\
\hline 179.0 & -39.0 & -27.0 & -9.7 & -40.1 & 1. & -20.8 & 294 & 1 & -12.0 & 28 & 1.8 & 0.00 \\
\hline 179.0 & -39.5 & -3 & -6 & -44.0 & 1. & -22.4 & 296 & 1 & -11.7 & 2 & 2.2 & 0.00 \\
\hline 179.0 & -40.0 & -40.2 & -4.1 & -44.5 & 1.4 & -22.0 & 297 & 0.1 & -10.3 & 298 & 3.6 & 0.00 \\
\hline 179.0 & -40.5 & -43.7 & -2.1 & -45.3 & -1.2 & -19.7 & 297 & -2.1 & -8.6 & 298 & 8.7 & 0.00 \\
\hline
\end{tabular}


Table B.11: Full results for Region 5 (southern and eastern North Island) calculated by applying the Flesch et al. [2001] stress method to the GPS-derived velocity model.

\begin{tabular}{|c|c|c|c|c|c|c|c|c|c|c|c|c|}
\hline Long. & Lat. & $v_{x}$ & $v_{y}$ & $\Gamma$ & $\tau_{e x}$ & $\tau_{c p}$ & $\tau_{a z}$ & $\tau_{G e x}$ & $\tau_{G c p}$ & $\tau_{G a z}$ & $\eta$ & Mis. \\
\hline 173.5 & -40.0 & -2.0 & -0.0 & -11.3 & -2.7 & -6.6 & 272 & 6.5 & -8.4 & 33 & 9.6 & 0.03 \\
\hline 174.0 & -40.0 & -3.3 & -0.4 & -14.2 & -2.7 & -7.6 & 273 & 6.0 & -8.9 & 34 & 8.0 & 0.03 \\
\hline 174.0 & -40.5 & -5.5 & -0.3 & -21.0 & -4.0 & -9.3 & 286 & 4.1 & -7.8 & 28 & 6.3 & 0.05 \\
\hline 174.5 & -40.0 & -4.7 & -1.0 & -16.4 & -2.8 & -8.1 & 278 & 5.1 & -8.4 & 33 & 10.9 & 0.10 \\
\hline 174.5 & -40.5 & -8.5 & -1.5 & -21.5 & -3.6 & -10.1 & 290 & 3.4 & -7.3 & 26 & 5.7 & 0.09 \\
\hline 174.5 & -41.0 & -13.7 & -3.2 & -16.7 & -2.0 & -10.7 & 279 & 4.3 & -7.5 & 30 & 3.5 & 0.09 \\
\hline 175.0 & -40.0 & -5.3 & -2.4 & -17.9 & -3.0 & -7.5 & 287 & 5.1 & -7.6 & 29 & 11.7 & 0.55 \\
\hline 175.0 & -40.5 & -11.6 & -3.2 & -21.0 & -3.4 & -9.4 & 293 & 4.2 & -7.3 & 25 & 4.5 & 0.11 \\
\hline 175.0 & -41.0 & -18.5 & -5.6 & -11.3 & -1.3 & -9.8 & 284 & 5.3 & -6.9 & 27 & 2.7 & 0.08 \\
\hline 175.0 & -41.5 & -30.0 & -9.3 & -14.7 & -0.6 & -13.0 & 279 & 3. & -6.1 & 26 & 3.4 & 0.04 \\
\hline 175.5 & -40.0 & -4.3 & -5.0 & -11.3 & -2.0 & -5.9 & 306 & 6. & -6.0 & 2 & 5.1 & 0.26 \\
\hline 175.5 & -40.5 & -15.6 & -5.9 & -15.9 & -3.2 & -8.0 & 302 & 5.7 & -7.3 & 23 & 2.9 & 0.14 \\
\hline 175.5 & -41.0 & -24.8 & -9.4 & -10.3 & -1.3 & -10.1 & 29 & 5 & -6 & 22 & 3.5 & 0.07 \\
\hline 176.0 & -39.5 & -0.6 & -8.0 & 11.3 & 2.6 & -1.2 & 297 & 8. & -2.4 & 27 & 4.3 & 0.67 \\
\hline 176.0 & -40.0 & -8.4 & -8.5 & -5.1 & 0.0 & -6.1 & 315 & 6. & -4.0 & 18 & 2.2 & 0.10 \\
\hline 176.0 & -40.5 & -20.8 & -9.9 & -14.6 & -1.8 & -9.3 & 311 & 4.9 & -5.7 & 18 & 4.5 & 0.12 \\
\hline 176.0 & -41.0 & -26.9 & -10.8 & -17.7 & -1.0 & -13.7 & 300 & 2.3 & -4.9 & 4 & 6.2 & 0.03 \\
\hline 176.5 & -39.0 & 2.7 & -11.9 & 15.7 & 5.2 & -0.4 & 82 & 9.8 & -3.4 & 48 & 5.3 & 0.27 \\
\hline 176.5 & -39.5 & -2.3 & -12.5 & -0.0 & 1.3 & -3.7 & 295 & 5 & -2.1 & 32 & 2.2 & 0.03 \\
\hline 176.5 & -40.0 & -11.0 & -12.0 & -14.2 & -0.3 & -8.7 & 313 & 3. & -2.9 & 13 & 8.2 & 0.24 \\
\hline 176.5 & -40.5 & -21.5 & -13.5 & -21.8 & -1.0 & -13.1 & 311 & 1.3 & -4.0 & 9 & 7.6 & 0.18 \\
\hline 177.0 & -38.5 & 2.1 & -14.9 & 17.6 & 7.3 & 0.1 & 82 & 10.5 & -3.4 & 66 & 5.9 & 0.15 \\
\hline 177.0 & -39.0 & 2.0 & -16.2 & 6.1 & 4.4 & -2.6 & 274 & 7.1 & -2.8 & 64 & 3.3 & 0.35 \\
\hline 177.0 & -39.5 & -4.4 & -16.1 & -11.1 & 0.6 & -7.0 & 295 & 1.4 & -1.4 & 54 & 3.4 & 0.02 \\
\hline 177.0 & -40.0 & -9.4 & -17.6 & -24.5 & -1.7 & -11.6 & 308 & -1.2 & -2.4 & 10 & 4.9 & 0.04 \\
\hline 177.5 & -38.5 & 1.6 & -17.1 & 14.2 & 6.5 & -0.9 & 86 & 9. & -4.2 & 73 & 5.1 & 0.36 \\
\hline 177.5 & -39.0 & -0.6 & -18.7 & 2.1 & 4.1 & -4.2 & 285 & 5. & -2.4 & 86 & 1.8 & 0.18 \\
\hline 177.5 & -39.5 & -7.7 & -17.4 & -11.8 & 1.1 & -8.6 & 300 & 1. & -1.5 & 292 & 1.8 & 0.00 \\
\hline 178.0 & -38.5 & -1.6 & -17.3 & 8.2 & 4.5 & -2.4 & 27 & 8.5 & -5.3 & 77 & 1.6 & 0.24 \\
\hline 178.0 & -39.0 & -4.1 & -18.5 & -1.6 & 3.4 & -6.5 & 290 & 4.7 & -3.3 & 278 & 1.0 & 0.05 \\
\hline
\end{tabular}


Table B.12: Full results for Region 6 (Taupo Volcanic Zone) calculated by applying the Flesch et al. [2001] stress method to the GPS-derived velocity model.

\begin{tabular}{ccccccccccccc}
\hline Long. & Lat. & $v_{x}$ & $v_{y}$ & $\Gamma$ & $\tau_{e x}$ & $\tau_{c p}$ & $\tau_{a z}$ & $\tau_{G e x}$ & $\tau_{G c p}$ & $\tau_{G a z}$ & $\eta$ & Mis. \\
\hline 175.5 & -37.5 & 1.7 & -1.2 & 6.4 & 2.6 & 1.8 & 35 & 5.7 & 0.9 & 4 & 2.0 & 0.02 \\
175.5 & -38.0 & 0.8 & -2.2 & 7.8 & 3.6 & 0.3 & 88 & 6.4 & -0.2 & 30 & 2.5 & 0.08 \\
175.5 & -38.5 & 1.2 & -3.6 & 17.0 & 5.9 & 0.1 & 88 & 9.4 & -1.9 & 43 & 2.8 & 0.17 \\
175.5 & -39.0 & 1.7 & -4.1 & 20.4 & 6.0 & 0.0 & 272 & 10.7 & -2.7 & 41 & 4.8 & 0.36 \\
175.5 & -39.5 & -1.0 & -3.2 & 8.0 & 2.2 & -2.0 & 287 & 8.7 & -3.8 & 31 & 6.1 & 0.39 \\
176.0 & -37.5 & 4.4 & -1.8 & 10.8 & 4.0 & 0.9 & 1 & 6.1 & 1.7 & 346 & 4.8 & 0.06 \\
176.0 & -38.0 & 2.3 & -2.8 & 15.2 & 4.2 & 2.0 & 68 & 7.4 & 0.5 & 36 & 3.8 & 0.00 \\
176.0 & -38.5 & 4.4 & -5.1 & 19.2 & 6.4 & 1.0 & 73 & 10.7 & -2.6 & 44 & 3.1 & 0.05 \\
176.0 & -39.0 & 2.9 & -8.5 & 21.0 & 5.6 & 1.1 & 82 & 11.1 & -2.8 & 40 & 4.9 & 0.07 \\
176.5 & -38.0 & 2.8 & -2.8 & 18.9 & 5.2 & 2.6 & 77 & 8.2 & 0.2 & 60 & 2.5 & 0.10 \\
176.5 & -38.5 & 1.7 & -10.8 & 20.1 & 7.1 & 0.8 & 74 & 10.9 & -2.9 & 54 & 2.6 & 0.09 \\
177.0 & -38.0 & 2.9 & -13.6 & 14.9 & 6.3 & 1.1 & 81 & 9.3 & -2.5 & 74 & 2.9 & 0.20 \\
\hline
\end{tabular}

